\title{
Acoustofluidics: Theory and simulation of streaming and radiation forces at ultrasound resonances in microfluidic devices
}

\section{Bruus, Henrik}

Published in:

Acoustical Society of America. Journal

Publication date:

2009

Document Version

Publisher's PDF, also known as Version of record

Link back to DTU Orbit

Citation (APA):

Bruus, H. (2009). Acoustofluidics: Theory and simulation of streaming and radiation forces at ultrasound resonances in microfluidic devices. Acoustical Society of America. Journal, 125(4), 2592-2592.

\section{General rights}

Copyright and moral rights for the publications made accessible in the public portal are retained by the authors and/or other copyright owners and it is a condition of accessing publications that users recognise and abide by the legal requirements associated with these rights.

- Users may download and print one copy of any publication from the public portal for the purpose of private study or research.

- You may not further distribute the material or use it for any profit-making activity or commercial gain

- You may freely distribute the URL identifying the publication in the public portal

If you believe that this document breaches copyright please contact us providing details, and we will remove access to the work immediately and investigate your claim. 


\title{
Session 2aAAa
}

\section{Architectural Acoustics: Multiple Channel Systems in Room Acoustics}

\author{
Ning Xiang, Cochair \\ Architecture, Rensselaer Polytechnic Inst., Troy, NY 12180 \\ Boaz Rafaely, Cochair \\ Electrical and Computer Engineering Dept., Ben Gurion Univ., Beer Sheva, Israel
}

Chair's Introduction-8:00

8:05

2aAAa1. A multi-channel audio system based on the theory of integral equations. Filippo M. Fazi and Philip A. Nelson (Inst. of Sound and Vib. Res., Univ. of Southampton, Highfield, S0171BJ, Southampton, U.K., ff1@ isvr.soton.ac.uk)

The basics of a multi-channel audio system, which attempts the reproduction of a desired sound field, are presented. The system's hardware consists of a three-dimensional array of loudspeakers, and can be used in combination with a specially designed microphone array. The mathematical fundamentals on which this technique is grounded consist of the formulation of the problems as an integral equation. The loudspeaker signals are determined from the knowledge of the target sound field on the boundary of a given control volume. The solution to this inverse problem is computed performing a singular value decomposition of the integral operator involved. For some simple array geometries it is possible to calculate an analytical solution to the problem. A regularization method is applied, as required by the ill-posed nature of the inverse problem under consideration. Some insight into the physical meaning of the illposedness is given and some analogies to near-field acoustic holography are suggested. The effectiveness of the method proposed has been verified experimentally and some of the experimental results are presented. Finally, it is shown how this technique has been successfully applied to the design of a multi-channel auralization system for room acoustics.

\section{$8: 25$}

2aAAa2. Representation of the musical instruments directivity using dodecahedron loudspeakers. Gottfried Behler and Martin Pollow (Inst. of Tech. Acoust., RWTH Aachen Univ., 52056 Aachen, Germany, gkb@akustik.rwth-aachen.de)

Room-acoustical measurements in general are performed with omnidirectional sound sources. With respect to auralization, such an impulse response may not be ideal since it does not represent the situation playing an instrument in the room. To achieve the directivity of a real source (such as an instrument or human voice) with a technical sound source (a loudspeaker) requires either to copy the body and the surface velocity distribution of that particular source or to reproduce the directional pattern of the radiation using a multiple source configuration like a dodecahedron loudspeaker array with independent excitation of each transducer. The advantage of the latter method is obvious since one single source is able to provide different directivities by changing the excitation profile. To maintain the appropriate excitation of each individual transducer, different approaches can be made. The method described here uses spherical harmonics decomposition of the target radiation pattern and a subsequent calculation of the frequency dependent excitation coefficients for each transducer. The advantage of this method is a flexible and fast calculation delivering filters that can be used either for real time convolution or off-line processing of the measuring signals. To measure the instruments directivity an array with 32 microphones is used.

\section{$8: 45$}

2aAAa3. Considering modal aliasing in the implementation of an acoustic echo canceller in the wave domain. Martin Schneider and Walter Kellermann (Multimedia Commun.s and Signal Processing, Univ. Erlangen-Nuremberg, Cauerrstr 7, 91058 Erlangen, Germany, schneider,wk@LNT.de)

Traditional multichannel acoustic echo cancellation applied to wave field synthesis systems is computationally extremely expensive due to the large number of channels to be identified. By using wave domain adaptive filtering (WDAF) one is able to choose an approximation which neglects the interaction (exchange of energy) between different modes. This reduces the number of necessary adaptive filter coefficients drastically. Furthermore, it allows to use computationally relatively inexpensive single-channel filter adaptation algorithms. Then, however, modal aliasing due to the limited resolution of the used microphone array becomes a significant problem since it constitutes an interaction between different modes with respect to the microphone signals. The effects of modal aliasing on the adaptation behavior of a WDAF-based echo cancellation system are analyzed and a modified wave domain representation of the synthesized wave field is proposed which reflects the limited resolution of the microphone array. 
2aAAa4. Spherical harmonic beamforming for room acoustic analysis. Gary W. Elko and Jens Meyer (mh acoustics LLC, 25A Summit Ave., Summit, NJ 07901)

We describe the potential of using a spherical beamforming microphone array to investigate the spatial correlation of sound fields in rooms. We are building spherical microphone arrays consisting of many acoustic pressure sensors mounted appropriately on the surface of a rigid sphere. Our associated spherical eigenbeamformer decomposes the sound-field into spatially orthonormal spherical harmonics up to third-order. We refer to the signals from the eigenbeamformer as eigenbeams. All eigenbeams have phase centers at the physical center of the array. Due to the orthonormal property of the eigenbeamformer, a diffuse field ideally results in zero correlation between the eigenbeams. Therefore, by measuring the cross-correlation between the eigenbeam signals, one can investigate the proximity to the isotropy (or "diffuseness") of the sound-field. Simultaneously, the underlying eigen-beam patterns can be steered without effecting the orthonormality property. How the cross-correlation function changes with general orientation of the eigenbeams is another potential measure for sound field diffuseness in rooms. We will show some real room measurements demonstrating the potential usefulness of this approach.

\section{9:25}

2aAAa5. Imaging room acoustics with the audio camera. Adam O’Donovan, Ramani Duraiswami, Nail A. Gumerov, and Dmitry N. Zotkin (Perceptual Interfaces and Reality Lab., Dept. of Comput. Sci. Univ. of Maryland, College Park, MD 20742)

Using a spherical microphone array and real time signal processing using a graphical processing unit (GPU), an audio camera has been developed. This device provides images of the intensity of the sound field arriving at a point from a specified direction to the spherical array. Real-time performance is achieved via use of GPUs. The intensity can be displayed integrated over the whole frequency band of the array, or in false color, with different frequency bands mapped to different color bands. The resulting audio camera may be combined with video cameras to achieve multimodal scene capture and analysis. A theory of registration of audio camera images with video camera images is developed, and joint analysis of audio and video images performed. An interesting application of the audio camera is the imaging of concert hall acoustics. The individual reflections that constitute the impulse response measured at a particular seat may be imaged, and their spatial origin determined. Other applications of the audio camera to people tracking, noise suppression, and camera pointing are also presented. [Work partially supported by NVIDIA and the VA.]

\section{9:45}

2aAAa6. Proposed method to measure the diffusion coefficient. Peter D'Antonio (651-C Commerce Dr., Upper Marlboro, MD 20774,pdantonio@rpginc.com)

A method to measure the uniform diffusion coefficient has been published as an AES Information Document [AES-4id-2001, JAES, Vol. 9, pp. 148-165 (March 2001)]. The method utilizes 37 fixed pressure zone microphones separated by 5 degrees located on a $1 \mathrm{~m}$ semicircle and a loudspeaker located on a $2 \mathrm{~m}$ concentric semicircle. A sample array sits at the origin. Under computer control 37 impulse responses are collected for the sample, background without sample, and a reference flat panel of similar size. The data reduction procedure will be reviewed to include all developments since the 2001 publication. In addition, experimental data will be presented along with the current presentation format. Currently the method is in the final stages of review for incorporation as Part 2 of ISO 17497-1, which describes the procedure to measure the random incidence scattering coefficient. A review and comparison between the scattering and diffusion coefficients will also be presented. A summary of this research can be found in the reference text [T. J. Cox and P. D'Antonio, Acoustic Absorbers and Diffusers: Theory, Design and Application, Spon Press 2004, second edition in press].

\section{0:05-10:15 Break}

\section{0:15}

2aAAa7. Analysis of in situ acoustic absorption using a spherical microphone array. Jonathan Rathsam and Boaz Rafaely (Dept. of Elec. and Comp. Eng., Ben-Gurion Univ. of the Negev, Beer-Sheva, Israel 84105, jrathsam@gmail.com)

Acoustic absorption of materials typically depends on the characteristics of the incident sound field. Differences in the incident sound field between one laboratory and another, or between the laboratory and in situ, may therefore lead to differences in the measured absorption for the same material. To overcome this problem, a new method is proposed to measure acoustical absorption using a spherical microphone array. With this method one measures simultaneously the acoustic absorption as well as the incident sound field. The incident sound field and the reflected sound field are separated by the spherical microphone array using spatial filtering, or beamforming. The acoustic absorption is then determined from the amplitudes of the incident and the reflected fields. The measurement method may also be useful for verifying sound field diffusivity in a reverberation chamber when measuring diffuse-field absorption. Furthermore, absorption coefficients measured under in-situ sound fields may yield more accurate computer-based sound field simulations than diffuse-field absorption coefficients when the in-situ sound field is not diffuse. [Work supported by the National Science Foundation.]

\section{$10: 35$}

2aAAa8. Speech intelligibility improvement in rooms using microphone arrays. Michael R. Stinson (Inst. for Microstructural Sci., Natl. Res. Council of Canada, Ottawa, ON K1A 0R6 Canada), Bradford N. Gover (Inst. for Res. in Construction, Natl. Res. Council of Canada, Ottawa, ON K1A 0R6 Canada), and Gilles A. Daigle (Natl. Res. Council of Canada, Ottawa, ON K1A 0R6 Canada)

Microphones arrays reduce the effects of reverberation and background noise as compared to a single microphone. The resulting improvement in speech intelligibility in rooms is investigated here, following the approach discussed by Houtgast et al. [Acustica 46, 60-72 (1980)]. Room characteristics (volume, reverberation time), the background noise spectrum, and the source-receiver separation are used to compute a modulation transfer function. This function is input into the procedure described in IEC standard 60268-16(2003) 
to arrive at an objective measure of speech intelligibility, the speech transmission index (STI). Doing this for both omnidirectional and microphone array receivers, the improvement in intelligibility is determined. The signal source has the spectral shape of a human talker, as given in the IEC standard; the background noise spectrum is based on measurements made in a restaurant. The benefit of using microphone arrays is discussed for various conditions.

10:55

2aAAa9. Inter-aural cross correlation in a sound field represented by spherical harmonics. Boaz Rafaely and Amir Avni (Dept. of Elec. and Comput. Eng., Ben-Gurion Univ. of the Negev, Beer Sheva 84105, Israel)

Spaciousness is an important acoustic feature of concert halls. An accepted measure for spaciousness is the inter-aural crosscorrelation (IACC), which employs the transfer function from a source in the hall to the two ears of a listener, the latter referred to as head-related transfer functions (HRTF). Recently, spherical microphone arrays have been studied for room acoustics analysis and music recordings. As these arrays typically use a finite number of microphones, they may not be able to capture the spatial information required for complete spatial analysis or for sound reproduction with realistic spaciousness. This study proposes the use of spherical harmonics representations for both the HRTF data and the sound field data, facilitating IACC analysis for sound fields represented by a finite order in the spherical harmonics domain. The effect of limited spherical harmonics order on the spaciousness of various sound fields is finally studied using simulated and measured data.

\section{Contributed Papers}

11:15

2aAAa10. A new method for the measurement of directional sound arrivals in rooms. Brian T. Thornock (Acoust. Res. Group, Dept. of Phys., Brigham Young Univ., N-283 ESC, Provo, UT 84602, brianthornock@gmail.com) and Timothy W. Leishman (Brigham Young Univ., Provo, UT 84602)

In room acoustics, directional measurements have the ability to more fully detail room effects than common nondirectional measurements. The extraction of directional information has been a subject of considerable interest for years and has resulted in the development of several interesting approaches to the problem. However, past methods have often suffered from inaccuracies, difficult implementation, lengthy procedures, extensive computational requirements, or the need for large amounts of equipment. A practical new method will be presented that extracts directional information of sound arrivals through a single multichannel measurement and a new crosscorrelation technique. A direct comparison of numerical simulations will demonstrate that the method is more accurate than other well-established methods. Advantages and limitations of the new approach will be discussed.

\section{1:30}

2aAAa11. Microphone array beamforming with near-field correlated sources. Jonathan L. Odom (Dept. of Elec. and Comput. Eng., Duke Univ., CIEMAS 2410B, Durham, NC 27708, jlo11@ duke.edu) and Jeffery Krolik (Duke Univ., Durham, NC 27708)

As the price of omnidirectional microphone arrays has fallen, new applications have emerged for large room audio capture. Further, large room acoustics use loudspeakers, which create correlated sources and severely limit the use of optimum beamformers. Most sources are in the near field due to the large wavelength of speech. Widrow and Kailath's work on correlation in sonar and radar assumed far field, but it cannot be directly applied to near-field acoustics. A new beamforming method has been devel- oped, which incorporates near field and correlation. The source bearings are estimated and an uncorrelated model is formed. A better DOA estimator was developed for the near-field correlated case limited by the width of the mainlobe. The new beamformer is able to null strong, perfectly and nearly perfectly correlated signals using the uncorrelated model and minimum variance distortionless response beamforming.

\section{$11: 45$}

2aAAa12. Prediction and measurement of reverberation increase from electroacoustic architecture systems. Roger Schwenke (Meyer Sound Labs., 2832 San Pablo Ave., Berkeley, CA 94702, rogers@meyersound.com), Steve Ellison (Meyer Sound Labs., Sierra Madre, CA 91024), and Mark Poletti (Industrial Res. Ltd., Lower Hutt, New Zealand)

Electroacoustic architecture (EA) systems increase the reverberation time (RT) of rooms by regenerating the physical reverberation of the room, and/or adding electronic reverberation to the direct sound. In regenerative systems, the amount of regeneration is controlled by the loop gain of the system. Increasing loop gain is analogous to reducing the effective absorption in the room. The RT of the electronic reverberator is analogous to the decay time of a physically coupled room, and, therefore, analogous to adding cubic volume to the room. As the electronic RT is increased, there is a point above which the resulting reverberation will begin to be double sloped. This point depends on the gain. The higher the gain, the higher the electronic RT before the resulting reverberation double slopes. In previous work, the gain before feedback of EA systems has been predicted in a statistical approach based on the bulk properties of the room such as RT and cubic volume. This, in turn, can be used to predict the maximum possible power gain and RT increase before double sloping. In this paper, we measure systems in use in the field and compare the achieved performance with predictions. 


\title{
Session 2aAAb
}

\section{Architectural Acoustics and the National Council of Acoustical Consultants: Student Design Competition}

\author{
Robert C. Coffeen, Chair \\ School of Architecture and Urban Design, Univ. of Kansas, Lawrence KS 66045
}

\begin{abstract}
The Technical Committee on Architectural Acoustics of the Acoustical Society of America, the National Council of Acoustical Consultants, and the Robert Bradford Newman Student Award Fund are sponsoring the 2009 Student Design Competition that will be professionally judged at this meeting. The purpose of this design competition is to encourage students enrolled in architecture, engineering, physics, and other university curriculums that involve building design and/or acoustics to express their knowledge of architectural acoustics and noise control in the design of a facility in which acoustical considerations are of significant importance.

This competition is open to undergraduate and graduate students from all nations. Submissions will be poster presentations that demonstrate room acoustics, noise control, and acoustic isolation techniques in building planning and room design. The submitted designs will be displayed in this session and they will be judged by the panel of professional architects and acoustical consultants. An award of USD \$1250.00 will be made to the entry judged "First Honors." Four awards of USD $\$ 700.00$ will be made to each of the four entries judged "Commendation."
\end{abstract}

TUESDAY MORNING, 19 MAY 2009

GALLERIA NORTH, 8:00 TO 11:55 A.M.

\section{Session 2aAB}

\section{Animal Bioacoustics and Acoustical Oceanography: Autonomous Remote Monitoring Systems for Marine Animals I}

\author{
Marc O. Lammers, Chair \\ Hawaii Inst. of Marine Biology, Kailua, HI 96734 \\ Chair's Introduction-8:00 \\ Invited Papers
}

8:05

\begin{abstract}
2aAB1. A survey of remote underwater autonomous acoustic recorders. Whitlow W. L. Au, Marc O. Lammers (Hawaii Inst. of Marine Biology, Univ. of Hawaii, P.O. Box 1106, Kailua, HI 96734), and Michael F. Richlen (Univ. of Hawaii Honolulu, HI 96734)

There has been a proliferation of different types of remote underwater autonomous acoustic recorders in recent years. Many were designed for a specific purpose to measure sounds from specific organisms. Others were designed for general application to encompass a wide variety of sounds. Many have fancy acronyms such as ARP, BPROBE, DTAG, EAR, EARS, HARP, MARU, PALs, RUDAR, and TPOD, to name a few. All have essentially the same subsystems consisting of a hydrophone and amplifier assembly, an analogto-digital converter, data storage, and a microcontroller to handle the data acquisition and storage operations and to control the overall functioning of the unit. The differences are in the bandwidth of the system, amount of storage available, amount of power required, and duration of deployment possible. Some can be purchased commercially, some can only be leased, while others can only be used in a collaborative research project. The prices also vary considerably. Some have been used for many years while others have come into play within the last few years. The sum total of all these acoustic recorders have contributed tremendously to our understanding of the marine ecosystem in ways that we could not have imagined several years ago.
\end{abstract}


2aAB2. Results and insights from operational acoustic monitoring networks. Christopher W. Clark (Bioacoust. Res. Prog., Cornell Univ., 159 Sapsucker Woods Rd., Ithaca, NY 148504), William T. Ellison (Marine Acoust., Inc., Middletown, RI 02842), Brandon L. Southall (Natl. Marine Fisheries Svce., Silver Spring, MD 20910-6233), Adam Frankel (Marine Acoust., Inc., Middletown, RI 02842), Dimitri Ponirakis, Christopher Tremblay, Ann Warde, and Eric Spaulding (Cornell Univ., Ithaca, NY 148504)

Data from two acoustic monitoring networks operating off New England in an area frequented by whales reveal acoustic features of those habitats. These seafloor and moored systems continuously sample the acoustic environment, and resultant data provide mechanisms for mapping, quantifying, and describing the spatio-spectral-temporal variability of the acoustic habitat over ecologically meaningful scales. By focusing on species-specific frequency bands used by fin, humpback, and right whales for long-range communication, we are beginning to measure the acoustic dynamics of their primary communication channels. Results reveal the extent to which different sources of sound in the ocean, both natural and man-made, influence the probability of whale communication. In some habitats with high rates of vessel traffic and high levels of vessel noise, the predicted area over which animals can communicate is reduced to a small proportion of what it would be under quiet conditions. The dynamics of this masking effect are highly variable and vary by species. When considered from a large-scale and behavioral ecological perspective, this reduction in acoustic habitat, as measured in terms of the proportional loss of communication space, likely represents a significant loss for species in which acoustic communication is known to serve critical biological functions.

\section{Contributed Paper}

$8: 45$

2aAB3. PAMGUARD: Semiautomated, open source software for real-time acoustic detection and localization of cetaceans. Douglas Gillespie (Sea Mammal Res. Unit, Univ. of St. Andrews, KY16 81B, Scotland, UK), David K. Mellinger (Oregon State Univ. and NOAA Pacific Marine Environ. Lab., Newport, OR 97365), Jonathan Gordon (Ecologic UK, Newport on Tay, Fife, DD6 8JH, UK), David McLaren, Paul Redmond, Ronald McHugh, Philip Trinder, Xiao-Yan Deng (Heriot-Watt Univ., Edinburgh, Scotland, UK), and Aaron Thode (Univ. of California, San Diego, La Jolla, CA 92093)

PAMGUARD is open-source, platform-independent software to address the needs of developers and users of Passive Acoustic Monitoring (PAM) systems. For the PAM operator-marine mammal biologist, manager, or
mitigator-PAMGUARD provides a flexible and easy-to-use suite of detection, localization, data management, and display modules. These provide a standard interface across different platforms with the flexibility to allow multiple detectors to be added, removed, and configured according to the species of interest and the hardware configuration on a particular project. For developers of PAM systems, an Application Programming Interface (API) has been developed which contains standard classes for the efficient handling of many types of data, interfaces to acquisition hardware and to databases, and a GUI framework for data display. PAMGUARD replicates and exceeds the capabilities of earlier real time monitoring programs such as the IFAW Logger Suite and Ishmael. Ongoing developments include improved real-time location and automated species classification. [PAMGUARD funded by the OGP E\&P Sound and Marine Life project.]

\section{Invited Papers}

\section{9:00}

2aAB4. Software for bioacoustic analysis of passive acoustic data. David K. Mellinger (Cooperative Inst. for Marine Res. Studies, Oregon State Univ. and NOAA Pacific Marine Environ. Lab., 2030 SE Marine Sci. Dr., Newport, OR 97365), Douglas Gillespie (Univ. of St. Andrews, KY16 81B, Scotland, UK), Harold Figueroa (Cornell Univ., Ithaca, NY 14850), Kate Stafford (Univ. of Washington, Seattle, WA 98105), and Tina Yack (NOAA Southwest Fisheries Sci. Ctr., La Jolla, CA 92038)

Software analysis systems comprise an important stage in passive acoustic research. Here we compare and evaluate three animal acoustic analysis software systems: Ishmael, PAMGUARD, and XBAT. These packages are compared and evaluated for their capabilities at some of the common tasks in animal sound analysis: recording, display, detection, classification, measurement, localization, and tracking of animal sounds. They are also compared for their extensibility (how easy is it, say, to add a new detection algorithm), their ease of use (how hard is the software to learn to use, how quick to use it once one knows how), their hardware interfacing (what types of sound acquisition hardware can they receive sound from), their sound file interfaces (can they read everyone's sound files), their documentation, and their software interfaces (how well do they share data with other programs, other methodologies, like visual surveys). Special features of each system will also be discussed.

\section{9:20}

2aAB5. Passive acoustic monitoring of marine ecosystems in the Pacific Islands Region. Marc O. Lammers (Joint Inst. for Marine and Atmospheric Res., NOAA-CRED, 1125B Ala Moana Blvd., Honolulu, HI 96814, lammers@ hawaii.edu), Kevin Wong, Russell E. Brainard (NOAA Fisheries, Honolulu, HI 96814), Whitlow W. L. Au (Univ. of Hawaii, Kailua, HI 96734), and Pollyanna Fisher-Pool (Joint Inst. for Marine and Atmos. Res., Honolulu, HI 96814)

Monitoring the changing state of marine habitats is a challenging task that is exacerbated when the habitats in question are in remote locations. Passive acoustic monitoring is sometimes the best, if not the only, means of gauging levels of biological and anthropogenic activities in such areas. Since 2006, the Pacific Islands Fisheries Science Center, in partnership with the University of Hawaii, has been building a network of long-term acoustic monitoring stations across the Pacific Islands Region using Ecological Acoustic Recorders (EARs). The network is currently composed of 29 long-term monitoring stations and spans the Hawaiian Archipelago, American Samoa, the Line Islands, Johnston Atoll, Wake Atoll, Guam, and the Commonwealth of the Northern Mariana Islands. EARs are diver-deployed and refurbished by local partners and/or during annual or biennial research cruises to deployment locations. A wide range of natural and anthropogenic acoustic signals are monitored, including sounds produced by invertebrates, fish, cetaceans, vessels, and surface weather events. The long-term trends in biological acoustic activity obtained through this network will be used to gauge the relative stability of the ecosystems associated with each location. Detections of vessels are common at many locations and provide a quantitative means of establishing levels of anthropogenic activity. 
2aAB6. Habitat use of beluga whales revealed by acoustic loggers. Manuel Castellote (Res. Dept., L'Oceanografic of Valencia, Spain, mcastellote@oceanografic.org), Nick Tregenza (Chelonia Ltd., United Kingdom), Ruth Leeney (Provincetown Ctr. for Coastal Studies, MA), José Antonio Esteban (Res. Dept., L'Oceanografic of Valencia, Spain), Gregory O'Corry-Crowe (Harbor Branch Oceanograph. Inst., FL), Bill Lucey (Yakutat Salmon Board, AK), Kathleen M. Stafford (Univ. of Washington, Seattle, WA), Kit M. Kovacs, Christian L. Lydersen (Norwegian Polar Inst., Norway), Rauno Lauhakangas (Helsinki Inst. of Phys., Finland), Vera Krasnova, Anton Chernetsky, Alexandr Agafonov, and Vsevolod Bel'kovich (Russian Acad. of Sci., Russia)

The detection of odontocete echolocation signals with acoustic loggers has proved to be a very effective way to monitor their distribution, seasonal patterns and habitat use. However, belugas (Delphinapterus leucas) have never been monitored using this methodology. In order to evaluate its viability, echolocation signals of captive belugas were analyzed and the performance of T-POD (Chelonia Ltd., UK) acoustic loggers in captivity and in the Arctic environment was tested. Results from captivity demonstrated the potential of beluga remote monitoring by T-PODs. Field tests were organized in different environmental conditions with different populations of belugas to ensure the validity and detection robustness of this method. T-PODs were deployed in Svalbard (Norway) in Spring 2007, in Yakutat (Alaska) in Spring 2008, and in Solovetsky (Russia) in Summer 2008. Instruments were deployed concurrently with visual observations and hydrophone recordings to compare presence/absence data from the three different methods. Results proved that T-PODs positively detected all the identified periods of beluga presence without false detections and revealed temporal distribution correlations with tide levels, feeding behaviors, and circadian rhythms in the echolocation activity. Results from this project indicate that beluga remote monitoring is feasible in the Arctic environment using acoustic loggers.

\section{0:00-10:20 Break}

\section{0:20}

2aAB7. Passive acoustical counting of odontocetes using towed and stationed platforms. Tomonari Akamatsu (Natl. Res. Inst. of Fisheries Eng., Fisheries Res. Agency, 7620-7 Hasaki, Kamisu, Ibaraki 314-0408, Japan), Ding Wang, Kexiong Wang, Songhai Li, Shouyue Dong (The Chinese Acad. of Sci., Wuhan, 430072, China), and Satoko Kimura (Kyoto Univ., 606-8501 Kyoto, Japan)

Counting the number of animals is essential for monitoring and management of marine mammals. Autonomous stereo acoustic event recorder (A-tag) to identify each sound source enabled to count phonating odontocetes in the wild. Finless porpoises were surveyed by towed A-tags in $1100 \mathrm{~km}$ stretch of Yangtze River from Wuhan to Shanghai. The calculated detection probability using acoustic method was approximately twice that for visual detection. The difference in detection probabilities between the two methods was caused by the large number of single individuals that were missed by visual observers. However, acoustics tended to underestimate group size due to the limited resolution of sound source bearing angles. The detection performance regarding stationary acoustic monitoring of this species was also examined. Three stereo acoustic data loggers were placed at different locations near the confluence of Poyang Lake and the Yangtze River. Acoustic monitoring confirmed the low density bottle neck of the population between the lake and the river that was consistent with visual observations. The passive pulse event monitoring was proved to be a practical and useful alternative to visual observations, especially for long-term and range-wide monitoring.

\section{Contributed Papers}

10:40

2aAB8. Acoustic tracking of whistling dolphins offshore of Southern California. Kaitlin E. Frasier, Sean M. Wiggins, and John A. Hildebrand (Scripps Inst. of Oceanogr., Univ. of California San Diego, Ritter Hall 200E, La Jolla, CA 92093-0205)

Dolphin whistles were recorded near San Clemente Island, offshore of Southern California, by four autonomous high-frequency acoustic recording packages (HARPs) deployed in a sea-floor array (dimensions: $2 \mathrm{~km} \times 2 \mathrm{~km}$ ) centered around the R/P FLIP. This array layout allowed localization of the calling animals using time difference of arrivals (TDOAs) from the same calls simultaneously recorded on the four instruments. Whistle TDOAs were calculated using cross-correlations of spectrograms. A matched-field leastsquares minimization procedure used whistle TDOAs to localize the animals. Successive localizations were combined to track movement over time, revealing local movements of presumably common dolphins (Delphinus spp.), typically during elevated nocturnal activity. These tracks, coupled with the animals' acoustic activity, provide insight into their behavior and could potentially be used to study their response to various stimuli including anthropogenic sources such as sonar. [This research is supported by CNO-N45.]

\section{0:55}

2aAB9. A real-time detection system for odontocete echolocation clicks in the low-energy processing environment of an acoustic glider. Holger Klinck (Cooperative Inst. for Marine Resour. Stud., and NOAA Pacific Marine Environ. Lab., Oregon State Univ. 2030 SE Marine Sci. Dr., Newport, OR 97365, Holger.Klinck@oregonstate.edu) and David K. Mellinger (Oregon State Univ., Newport, OR 97365)
This presentation will introduce a real-time system, the energy ratio mapping algorithm (ERMA), for detection of odontocete echolocation clicks. The system has been developed to run continuously over extended periods of time in low-energy processing environments such as acoustic gliders. The detector operates in the time domain and takes advantage of the species-specific differences in the power spectra and interclick interval of echolocation clicks to minimize the number of false positive detections. We will demonstrate the operation of ERMA using the clicks of beaked whales, family Ziphiidae, as example target species. To get information on the performance of ERMA, a comparison with more sophisticated and already established detection systems was conducted. Preliminary results indicate that the developed method is a promising tool to reliably detect echolocation clicks emitted by beaked whales in real time. On-going work is focused on implementation of the detector in the acoustic seaglider developed by the Applied Physics Laboratory of the University of Washington (APL-UW) and the use of this platform for autonomous passive acoustic monitoring and mitigation of beaked whales and other odontocete species.

\section{1:10}

2aAB10. Autonomous seafloor recorders and autodetection buoys to monitor whale activity for long-term and near-real-time applications. Christopher Tremblay, Tom Calupca, Christopher W. Clark, Matt Robbins, Eric Spaulding, Ann Warde (Bioacoust. Res. Prog., Cornell Univ., 159 Sapsucker Woods Rd., Ithaca, NY 148504, cjt35@ cornell.edu), John Kemp, and Kristopher Newhall (Woods Hole Oceanograph. Inst., Woods Hole, MA 02543)

Biologists and engineers from Cornell have installed arrays of autonomous seafloor recorders in multiple ocean habitats. These systems are de- 
signed to continuously monitor for the occurrence of endangered whales, particularly right whales; provide critical data on whale seasonal occurrence, distribution and relative numbers; and evaluate potential noise impacts from manmade activities and commercial shipping. Recent studies have been conducted in Massachusetts Bay around commercial LNG ports, the Arctic Ocean at seismic study locations, and in New York waters extending to the shelf edge. In combination with Cornell-developed MATLAB- and JAVA-based software applications, these seafloor recorders have been utilized to evaluate seasonal whale presence, calculate levels of commercial shipping and construction noise, and locate and track the movements and behaviors of individual whales under different acoustic conditions. Cornell and ocean engineers from the Woods Hole Oceanographic Institution have installed an operational network of autodetection buoys off New England to provide near-real-time data on right whale presence to transiting LNG vessels, government agencies, and public entities. When combined, the seafloor and autodetection buoy systems provide a powerful mechanism for understanding whale behavioral ecology and describing their acoustic habitats, while mitigating the risks from ship strike and noise exposure.

\section{$11: 25$}

2aAB11. Passive acoustic survey of bowhead whales in the Chukchi Sea. Julien Delarue, Marjo Laurinolli, and Bruce Martin (JASCO Res., Ste. 432, 1496 Lower Water St., Halifax, NS B3J 1R9, Julien.Delarue@jasco.com)

Western Arctic bowhead whales (Balaena mysticetus) transit through the Chukchi Sea in spring and fall during their seasonal migration between their winter grounds in the Bering Sea and their summer feeding grounds in the Beaufort Sea, where their presence is well documented. Comparatively little is known about their use of the Chukchi Sea in summer and winter. Here results are presented from a long-term passive acoustic monitoring program using a multi-recorder array deployed from late July 2007 through July 2008 in this area. Bowhead calls were recorded intermittently between August 2007 and January 1, 2008 and again from May 27, 2008 until the end of the study. Of particular interest is the detection of songs in late fall-early winter, which had previously only been recorded during the northward spring migration. They were compared to songs recorded off West Greenland during the previous mating season and found to be different. This is the first evidence of geographic variation of bowhead songs. Overall these detections provide new information on bowheads' spatiotemporal use of the Chukchi Sea. The successes of the study also highlight the effectiveness of autonomous underwater acoustic recorders for marine mammal surveys in remote locations.

\section{1:40}

2aAB12. A high-resolution pressure tag for ocean depth measurements. Prajas John, Jaison Peter, Adrine Antony Correya, M. H. Supriya, and P. R. Saseendran Pillai (Dept. of Electron., Cochin Univ. of Sci. and Technol., Cochin- 682022, India)

Data Storage Tags form an important class of electronic tags, used for studying the migratory patterns of highly migratory marine species. In such tags, the parameters to estimate the position of the species are recorded in the tag memory and are retrieved subsequently upon recapturing the tag. The vertical and spatial migration of such marine species can be analyzed in detail using the pressure measurements made by the tags. The migratory behavior of marine species can be attributed to the light intensity variations and temperature profile as well as the search of food and suitable environmental conditions. The depth measurements can be made more accurate, if the resolution of the pressure sensor is significantly high. An absolute pressure measurement with a resolution of 1.2 mbar (approx. $1.2 \mathrm{~cm}$ in depth measurements) has been achieved with a pressure tag, capable of recording pressures at preset time intervals. Conversion of the hydrostatic pressure measurements into linear depth variation is carried out using the well-known equations. Pressure measurements were carried out using the pressure tag in the laboratory conditions as well as in the open ocean and the depth variations were computed using a user-friendly interactive software.

TUESDAY MORNING, 19 MAY 2009

BROADWAY I/II, 8:15 TO 11:20 A.M.

\title{
Session 2aAO
}

\section{Acoustical Oceanography and Animal Bioacoustics: Acoustic Backscattering from Marine Life in the Ocean}

\author{
Christopher D. Jones, Chair \\ Applied Physics Lab., Univ. of Washington, Seattle, WA 98105
}

Chair's Introduction-8:15

\section{Contributed Papers}

\section{$8: 20$}

2aA01. Theoretical models for imaging distributed fish groups in an ocean waveguide with wide-area sonar including multiple scattering. Mark Andrews and Purnima Ratilal (Northeastern Univ., 409 Dana Res. Ctr., Boston, MA 02115)

Wide area sonar has been shown to be a useful tool for instantaneously imaging shoals of fish distributed over large areas on the continental shelf. Numerical models are applied to simulate the active imaging system and determine the statistics of the received matched filtered intensity scattered off remote fish groups in the ocean waveguide environment. The model includes multiple scattering of the dense fish groups and uses a rangedependent acoustic model to simulate the ocean environment. The numerical model results are compared to analytic models, which make a single scattering assumption. We find the conditions for when multiple scattering is important and show that it is a function of both the fish density and mean target strength for a given distribution of fish. We use Monte Carlo simulations to explore the effects on the statistics of the received matched filtered intensity for an ocean waveguide randomized by internal waves. The model can be used to infer fish densities and abundances in wide area sonar imagery as well as provide error analysis of these estimates.

\section{8:35}

2aAO2. Atlantic herring low frequency target strength estimation from ocean acoustics wageguide remote sensing (OAWRS) data in the Gulf of Maine over 10 days of observation. Duong Duy Tran, Zheng Gong, Nikhila Srikanth, Mark Andrews, Purnima Ratilal (Dept. Elec. and Comput. Eng., Northeastern Univ., 360 Huntington Ave., Boston, MA 02115, tran.duo@neu.edu), and Nicholas Makris (MIT, Cambridge, MA 02139)

During the Gulf of Maine acoustic experiment in the fall of 2006, massive shoals of Atlantic herring were instantaneously imaged over wide areas using an ocean acoustics waveguide remote sensing (OAWRS) system during evening to midnight hours over a period of 10 days from Sept. 26 to Oct. 5. The low frequency target strength (TS) and abundances of the herring 
population have been estimated by correlating the OAWRS data with localized measurements from a conventional fish finding sonar (CFFS) for 3 days from Oct. 1-3 [Gong et al., J. Acoust. Soc. Am. 124, 2586 (2008)]. Here we provide an analysis of the low frequency TS of the herring population in the frequency range from 300 to $1500 \mathrm{~Hz}$ and abundances for the remaining 7 days of the experiment. The acoustic scattering from herring populations is highly frequency dependent and is well modeled using a resonant scattering model for swimbladder bearing fish. Here we compare the TS estimates and the neutral buoyancy depth for herring over the observation period of 10 days.

\section{8:50}

2aAO3. Midfrequency backscatter imaging of fish schools in a shallow water waveguide. Christopher D. Jones and Darrell R. Jackson (Appl. Phys. Lab., Univ. of Washington, Seattle, WA 98105, cjones@ apl.washington.edu)

In this experimental effort, field data and modeling are used to investigate backscatter imaging of schools of pelagic fish in a shallow water oceanic waveguide. Two-dimensional (2-D) circular images of an oceanic waveguide are created using a monostatic circular receiving array and vertical line array source $(12 \mathrm{kHz})$ deployed from a stationary ship. Horizontal multibeam images of a radial (depth integrated) cross section of the ocean are formed with targets and reverberation structure observed at ranges up to several $\mathrm{Km}$. Examples of waveguide images are presented for data taken in the Puget Sound of Washington state. In some instances, the backscatter from aggregations of fish was observed to be higher than the reverberation; in other cases, fish schools could not be resolved. Fish school locations were independently observed by vertical echo sounding. Preliminary experimental results are shown to illustrate the potential of resolving the 2-D horizontal structure of aggregations of fish using such methods. Modeling is used to interpret the effects of waveguide propagation on imaging. Operational issues related to using waveguide imaging for fisheries' research and signal processing issues related to waveguide imaging will be discussed. [Work funded by NOPP with support from ONR and NOAA-OE.]

\section{9:05}

2aAO4. Tracking fish in a school using a broadband split-beam system. Masanori Ito, Ikuo Matsuo (Dept. of Information Sci., Tohoku-Gakuin Univ., 2-1-1 Tenjinsawa, Izumi-ku, Sendai, 981-3193, Japan), Tomohito Imaizumi, Tomonari Akamatsu (Fisheries Res. Agency, Kamisu, Ibaraki, 314-0408, Japan), and Yasushi Nishimori (Furuno Electric Co., Ltd, Nishinomiya, 662-8580, Japan)

Split-beam echo-sounder has been an important tool to understand fish behaviors undersea. In the split-beam system, each position of these fishes in three-dimensional space was estimated from delays of echoes and differences of delays between receive channels. To estimate the positions with high spatial resolution, the split-beam system which can emit and receive the broadband signals was constructed. However, in the case of a school of fish, it is difficult to determine accurately delay times of these fish because the echoes are overlapped with one another. To solve this problem, the new method was proposed to estimate delay times of fish by using the onset information of echoes, that is, extracting the steep temporal changes of the envelope pattern computed from the echo waveform. In a water tank experiment, it is examined that a location of a sphere can be accurately estimated by using the proposed method. In the sea, the echoes from schools of fish were measured with this system. It is examined that each location of fish in the three-dimensional space can be estimated and the numbers of fish can be counted. The tracks of the fish can be computed by changes of the fish locations along pings.

\section{9:20}

2aA05. Broadband acoustic backscattering from live squid: Experiment and analysis. Wu-Jung Lee, Timothy K. Stanton, and Andone C. Lavery (Woods Hole Oceanogr. Inst., Dept. of Appl. Ocean Phys. and Eng., Woods Hole, MA 02543,wjlee@whoi.edu)

Squid are important organisms both ecologically and commercially Acoustic scattering techniques can provide synoptic data on their distribution and abundance, and have the advantage of being efficient compared with traditional net sampling methods. However, knowledge of the scatter- ing properties of squid is required to accurately convert the acoustic data into meaningful biological information. To better understand the scattering of sound by squid, and to facilitate future model development, a controlled laboratory backscattering experiment was conducted on live squid (Loligo pealeii) using broadband linear chirp signals $(45-105 \mathrm{kHz})$ with data collected over the full $360 \mathrm{deg}$ of orientation in the lateral plane, in 1-deg increments. The scattered spectra often showed significant structure over the frequency band available at different angles of orientation, in addition to high levels of ping-to-ping variability. Pulse compression signal processing techniques were also used, revealing the following dominant scattering features: (1) at normal incidence, the front and back interface of the animal were resolved and (2) at off-normal incidence, the anatomical features in the head region were found to dominate the scattering. This information can serve as the basis for an accurate acoustic scattering model for squid.

\section{9:35}

2aA06. Humboldt squid distribution in three-dimensional space as measured by acoustics in the Gulf of California. Robyn S. Matteson, Kelly J. Benoit-Bird (College of Oceanogr. and Atmospheric Sci., 104 COAS Admin. Bldg., Oregon State Univ., Corvallis, OR 97331, rmatteson@coas.oregonstate.edu), and William F. Gilly (Stanford Univ., Pacific Grove, CA 93950)

The Humboldt squid, Dosidicus gigas, is a voracious migratory predator, important prey for many species, and the target of an economically important fishery. However, information on the behavior, life history, and biomass of this species is limited. Current knowledge is based almost exclusively on fisheries records, which only contain nighttime observations and provide no information on squid distribution and behavior outside of heavily fished areas. Acoustic measurements have long been used to study fish. Development of this method for application to squid provides a noninvasive technique to assess squid numbers and sizes. This method was applied in March and April of 2007 and November of 2008 in the Gulf of California, with a focus on the Santa Rosalia region. Squid densities were as high as 25000 per $\mathrm{km}^{3}$. Larger numbers of squid were observed at shelf breaks relative to other areas. Squid exhibited a marked diel vertical migration, with daytime depths of 300-400 m, bathymetry allowing, and nighttime depths of 0-200 $\mathrm{m}$. In shallower coastal areas, the distribution of squid closely mirrored the shape of the bottom topography. This new technique is providing previously unattainable insights into the behavior and biology of this commercially and ecologically important squid species.

\section{9:50}

2aAO7. Acoustical and optical measurements provide evidence of ecological interactions in planktonic thin layers. Kelly J. Benoit-Bird and Timothy J. Cowles (College of Oceanic and Atmos. Sci., Oregon State Univ., 104 COAS Admin. Bldg., Corvallis, OR 97331, kbenoit@coas .oregonstate.edu)

Coincident physical, optical, and acoustical data were obtained in Monterey Bay, CA during an extensive study of planktonic layers. These concurrent data describing the distributions of phytoplankton, zooplankton, and small fish as well as gradients in the physical habitat were used to examine the interactions between vertically compressed plankton structures (thin layers), their consumers, and the local physical forces they experience. The steepness of the vertical gradient on the top versus the bottom of the plankton layer was correlated to the difference in the relative abundance of consumers above and below the layer. Phytoplankton layer gradients were steeper when more zooplankton were present on one side of the layer versus the other while zooplankton layers were more diffuse with a greater number of fish adjacent to one side of the layer than the other. Both layer types showed nearly symmetrical gradients when predators were in low abundance or absent. Differences in phytoplankton and zooplankton layer "shape" were not correlated with vertical gradients in shear or mixing potential surrounding layers. The consequences of trophic interactions within aggregations can be detected and assessed, and in the absence of strong physical gradients, grazers can play an important role in structuring plankton thin layers. 
10:20

2aAO8. Long-term broadband acoustic observations of zooplankton scattering layers in Saanich Inlet, British Columbia. Tetjana Ross (Dept. of Oceanogr., Dalhousie Univ., Halifax NS B3H 4J1, Canada; tetjana@dal.ca) and Gareth Lawson (Woods Hole Oceanograph. Inst., Woods Hole, MA 02543)

The application of broadband techniques to fish and zooplankton bioacoustics is showing potential to transform the field into one that is much more quantitative. This is because broadband techniques allow the use of the known spectra of organisms or nonbiological sources of scattering to distinguish between scatterers, allowing discrimination without the need for extensive groundtruthing. This makes it ideal for remote monitoring of fish or zooplankton assemblages, since continuous net-sampling is often not possible. An upward-looking $85-155 \mathrm{kHz}$ broadband sonar has been collecting data nearly continuously on the Victoria Experimental Network Under the Sea (VENUS) mooring in Saanich Inlet, British Columbia since March 2008. Saanich Inlet is known to have large populations of euphausiids, which create a strong acoustic scattering layer that migrates from depth to the surface and back each day. The thickness, timing, strength and spectral response of this layer is examined throughout the annual cycle and the feasibility of using this type of sonar to elucidate changing zooplankton assemblages (due to growth and changing species composition) is assessed.

10:35

2aA09. Temporal patterns of fish and mesozooplankton near the Columbia River plume. Amanda M. Kaltenberg (College of Oceanic and Atmospheric Sci., Oregon State Univ., 104 COAS Admin. Bldg., Corvallis, OR 97331, akaltenb@coas.oregonstate.edu), Kelly J. Benoit-Bird (Oregon State Univ., Corvallis, OR 97331), and Robert L. Emmett (NOAA Northwest Fisheries Sci. Ctr., Newport, OR 97365)

The distribution of the dominant fish species in the California Current System is seasonal, with the timing and variability of these distributions having significant ecological consequences. The objective of this study was to observe the seasonal timing of fish school presence and to characterize the temporal patterns their abundance. Fish and mesozooplankton were observed near the Columbia River plume using 200-kHz bioacoustic moorings deployed throughout the transition period from winter conditions to summer upwelling conditions in 2008. Sea surface temperature, sea surface salinity, and ocean current data were used to characterize the oceanographic habitat. Acoustic mooring data revealed that the seasonal timing of schools of $\mathrm{Pa}-$ cific sardine and northern anchovy were linked with temperature and persisted after a rapid increase in temperature occurred near the end of May. The abundance of fish schools and mesozooplankton at two mooring stations was highly variable with dramatic fluctuations occurring over a period of hours to days that were linked with ocean conditions. The use of stationary acoustic moorings in this study provided an effective tool to monitor fish abundance at high temporal resolution and provides insight into the timing and variability of fish populations that will improve ecosystem-based management decisions.
10:50

2aAO10. Pilot work using a codend video camera for improved acoustic interpretation of backscatter observed during fisheries acoustic surveys. Lisa A. Bonacci and W. Waldo Wakefield (NOAA, Fisheries, NWFSC, 2032 SE OSU Dr., Newport, OR 97365, Lisa.Bonacci@noaa.gov)

One challenging but crucial element of fisheries acoustic surveys is accurate groundtruthing of the echo return. Assignment and proportioning of areas of backscatter to the correct species or mix of species affects fish biomass calculations. Typically, survey groundtruthing is done by midwater trawls targeting a single scattering layer of interest. However, the depth at which each species is caught is unknown. Therefore, this method is problematic when multiple scattering layers are present. Small scatters are also missed, which may be important contributors to the echo intensity. To address these issues, we conducted pilot work using a video camera mounted in the codend of an open midwater trawl, which enabled us to look at several scattering layers during each tow. For comparison, we conducted tows with a closed net and no camera at the same location. We then examined each acoustic echogram and the corresponding tow in order to compare backscatter calculations attributable to our species of interest. We found that there were some differences in these measurements between the two types of tows. We conclude that this method appears to provide improved knowledge of acoustic backscatter observed during fisheries acoustic surveys and plan to integrate this method into future surveys.

\section{1:05}

2aAO11. Density and soundspeed measurements of various Bering Sea zooplankton. Joseph D. Warren, Joy N. Smith (School of Marine and Atmospheric Sci., Stony Brook Univ., Southampton, NY 11901, joe.warren@stonybrook.edu), and Patrick H. Ressler (NOAA Fisheries AFSC, Seattle, WA 98115)

Walleye pollock is a commercially and ecologically important species in the Bering Sea. Assessment of pollock stocks is conducted regularly using a combination of trawl net sampling and multiple frequency active acoustic echosounder surveys. In addition to backscatter from the species of interest, other marine life including several types of zooplankton and nekton can contribute substantial amounts of acoustic backscatter energy which can provide information regarding the abundance and distribution of these animals as well. Acoustic scattering models are used to estimate both the amount and frequency response of the acoustic energy scattered by the zooplankton, but these models require knowledge of various properties that describe the acoustically important characteristics of the zooplankton. We collected live zooplankton from the Bering Sea during June and July 2008. The morphology (spatial dimensions) and material properties (density and sound speed) of individual and groups of zooplankton (and some nekton) were measured. The taxa studied included euphausiids (Thysanoessa raschii, Thysanoessa inermis, Thysanoessa spinifera), copepods (Neocalanus sp.), amphipods (Themisto sp.), gastropod (Clione sp.), siphonophores, jellyfish, and squid. The results of our measurements with regard to acoustic scattering model development and use will also be discussed. 


\title{
Session 2aBB
}

\section{Biomedical Ultrasound/Bioresponse to Vibration: Biomedical Applications of Acoustic Radiation Force}

\author{
Mostafa Fatemi, Chair \\ Physiology and Biophysics, Mayo Clinic, Rochester, MN 55905
}

Chair's Introduction-8:00

\section{Contributed Papers}

\section{8:05}

2aBB1. Radiation force on spheres in helicoidal Bessel beams modeled using finite elements. David B. Thiessen, Likun Zhang, and Philip L. Marston (Phys. and Astron. Dept., Washington State Univ., Pullman, WA, 99164-2814, thiessen@wsu.edu)

Analysis of the scattering of sound by spheres centered on ordinary and helicoidal (higher-order) Bessel beams [P. L. Marston, J. Acoust. Soc. Am. 124, 2905-2910 (2008)] makes it possible to evaluate the radiation force on spheres centered on the beam in an ideal inviscid fluid. Potentially surprising results include cases where the radiation force is directed opposite the propagation direction of both types of beams [P. L. Marston (submitted for publication)]. For potential biomedical applications such as drug delivery, it would be necessary to know if a small transverse displacement of the sphere from the beam's axis causes a radiation force that pushes the sphere toward (or away from) the axis of the beam. Three-dimensional finite elements were applied to that problem. To trust FEM calculations of the radiation force with helicoidal beams, it was first necessary to verify that analytical values for the axial force are recovered in the on-axis helicoidal case since only the zero-order beam had been previously studied [D. B. Thiessen and P. L. Marston, J. Acoust. Soc. Am. 122, 3025 (2007)]. Cases were found where displacement of a sphere from the beam axis causes a transverse restoring force. [Supported by ONR and NASA.]

\section{$8: 20$}

2aBB2. Radiation torque on a sphere centered on an acoustic helicoida (vortex) Bessel beam. Likun Zhang and Philip L. Marston (Phys. and Astron. Dept., Washington State Univ., Pullman, WA 99164-2814, zhanglikun@wsu.edu, marston@wsu.edu)

Circularly polarized electromagnetic waves carry axial angular momentum and analysis shows that the axial radiation torque on an illuminated sphere is proportional to the power absorbed by the sphere [P. L. Marston and J. H. Crichton, Phys. Rev. A. 30, 2508-2516 (1984)]. Hefner and Marston [J. Acoust. Soc. Am. 106, 3313-3316 (1999)] proposed that a helicoida acoustic beam (an acoustic vortex) also carries axial angular momentum and noted that absorption of such a beam should also produce an axial radiation torque. In the present work the acoustic radiation torque on a sphere centered on an acoustic helicoidal Bessel beam is analyzed and the torque is predicted to be proportional to the ratio of the power absorbed as a result of the interaction of sound with the sphere to the acoustic frequency. The torque is also proportional to the topological charge of the beam. Depending on the beam helicity, the torque is parallel or anti-parallel to the beam axis The analysis uses a relation between the scattering and the partial wave coefficients for a sphere in a helicoidal Bessel beam [P. L. Marston, J. Acoust Soc. Am. 124, 2905-2910 (2008)]. [This research was supported in part by ONR and NASA.]

\section{$8: 35$}

2aBB3. Acoustic radiation force on a gas bubble in tissue. Yurii $A$ Ilinskii, Evgenia A. Zabolotskaya, and Mark F. Hamilton (Appl. Res. Labs., The Univ. of Texas at Austin, Austin, TX 78713-8029)
The motion of a gas bubble subjected to acoustic excitation in a soft elastic medium such as tissue was analyzed previously assuming the radiation force acting on the bubble is the same as in liquid [Ilinskii et al., J. Acoust. Soc. Am. 117, 2338 (2005)]. In the present work we discuss corrections to the acoustic radiation force for finite values of the shear modulus. The analysis is based on the Piola-Kirchhoff equation in Lagrangian coordinates, in which only the stress tensor is nonlinear, and the equation is solved by perturbation. In the linear approximation an analytical solution is obtained for the scattered acoustic wave. The nonlinear stress and full radiation force is calculated at the next order of approximation. For negligible shear modulus the result for a liquid is recovered. For small but finite shear modulus the resulting force differs from that for a liquid by a factor that depends on $k_{t} R$, where $R$ is bubble radius, $k_{t}=\omega / c_{t}$ the wavenumber, $\omega$ the angular frequency, and $c_{t}$ the shear wave propagation speed. For $k_{t} R>10$ the radiation force is practically the same as in liquid, but for $k_{t} R<10$ its value can be significantly different. [Work supported by NIH DK070618.]

$$
\text { 8:50 }
$$

2aBB4. Optimization of ultrasound radiation force source for shear wave dispersion measurements. Matthew W. Urban and James F. Greenleaf (Dept. of Physio. and Biomedical Eng., Mayo Clinic College of Medicine, 200 First St. SW, Rochester, MN 55905, urban.matthew@mayo.edu)

The objective of this study was to analyze and optimize the ultrasound radiation force source for creating shear waves for quantitative elasticity and viscosity measurements. A simulation model based on work by Bercoff $e t$ al., [IEEE Trans. Ultrason. Ferroelectr. Freq. Contr. 51(11), 1523-34 (2004)] was utilized to examine the shear wave propagation from a spatiotemporal impulsive force and the simulated radiation force generated from a linear array transducer. Parameters such as aperture size, F-number of the transducer, and medium shear elasticity and viscosity were varied to analyze how the shear wave amplitude and shape changes. The wave attenuation and group and phase velocities were evaluated. The aperture size and the F-number of the transducer affected the spatial distribution of the propagating shear wave significantly. The influence of the coupling term between the bulk and shear waves was analyzed spatially and temporally by evaluating parameters such as shear wave amplitude, wave attenuation, and the group and phase velocities. This simulation model provides insight for making shear wave propagation measurements from the perspective of optimizing the spatial and temporal characteristics of induced shear wave motion from an applied radiation force. [This work was supported in part by Grant EB002167 from NIH.]

\section{9:05}

2aBB5. Comparison of unconfined compression and spatially modulated ultrasound radiation force estimates of shear modulus. Stephen A. McAleavey, Erin Collins, Etana Elegbe, and Johanna Kelly (BME Dept., Univ. of Rochester, 309 Goergen Hall, Rochester, NY 14627, stephenm @ bme.rochester.edu)

Spatially modulated ultrasound radiation force (SMURF) [S. McAleavey et al., Ultrason. Imag. 29, 87-104, (2007)] is a novel method for ultrasonic estimation of the low-frequency shear modulus properties of elastic media. In this approach, radiation force with a known lateral magnitude variation is 
applied impulsively within a region of interest using short $(\sim 30 \mu s)$ bursts of ultrasound. The spatial frequency $k$ of the radiation force intensity variation is selectable and determined through beamforming. Application of the radiation force impulse gives rise to a low-frequency $(500-2000 \mathrm{~Hz}$ ) shear wave. The temporal frequency $\omega$ of this wave is measured using Doppler ultrasound methods. The modulus is estimated from the relationship $G=\rho(\omega k)^{2}$, where $\rho$ is the material density. To validate this method, the moduli of five samples of Zerdine (CIRS, Incorporated), an ultrasonically tissue-equivalent elastic material, were measured using conventional unconfined cyclic compression and SMURF implemented on a Siemens Antares scanner. The samples were cylindrical with diam $54 \mathrm{~mm}$ and height $25 \mathrm{~mm}$. The shear modulus of the samples ranged from $2.5-35 \mathrm{kPa}$. For all samples, the two methods agreed to within the estimated variation. An intrasample variation of $8 \%$ was observed for SMURF in the (assumed uniform) samples. [Work supported by NIH/NIBIB.]

\section{9:20}

2aBB6. Optical tracking of acoustic radiation force impulse-induced dynamics in a tissue-mimicking phantom. Richard Bouchard (Dept. of Biomedical Eng., Duke Univ., Box 90281, Durham, NC 27708, rrb@duke.edu), Jason Streeter (UNC-CH and NCSU, Chapel Hill, NC 27599), Mark Palmeri, Gregg Trahey (Duke Univ., Durham, NC 27708), and Paul Dayton (UNC-CH and NCSU, Chapel Hill, NC 27599)

Optical tracking was utilized to investigate the acoustic radiation force impulse (ARFI)-induced response in a translucent, tissue-mimicking phantom. Suspended $10-\mu \mathrm{m}$ microspheres were tracked axially and laterally at multiple locations throughout a microscope's field of view with $0.5-\mu \mathrm{m}$ displacement resolution, in both dimensions, and at frame rates (with an attached video camera) of up to $36 \mathrm{kHz}$. Induced dynamics was successfully captured before, during, and after the ARFI excitation at depths of up to 4.8 $\mathrm{mm}$ from the phantom's proximal boundary. Results are presented for tracked axial and lateral displacements resulting from on-axis and off-axis (i.e., shear wave) acquisitions; these results are compared to matched finite element method modeling and independent, ultrasonically based empirical results. A shear wave reflection, generated by the proximal boundary, consistently produced an artifact in tracked displacement data later in time (i.e., after the initial ARFI-induced displacement peak). This new tracking method provides high-frame-rate, two-dimensional tracking data and thus could prove useful in the investigation of complex ARFI-induced dynamics in controlled experimental settings. [This work was supported in part by a National Science Foundation Graduate Research Fellowship.]

\section{9:35}

2aBB7. Acousto-magnetometry: A new vibrometery based on acoustic radiation force and magnetic measurement. Antonio Adilton O. Carneiro, Oswaldo Baffa (Departamento de Fisica e Matematica, Universidade de São Paulo, Ribeirao Preto, SP 14040-901 Brazil), Glauber T. Silva (Universidade Federal de Alagoas, Maceio, AL, 57072-970 Brazil), and Mostafa Fatemi (Mayo Clinic and College of Medicine, Rochester, MN 55905)

This work introduces a new method based on acoustic radiation force and magnetic measurement for interrogating the mechanical properties of fluid and biological tissues. In this method, ultrasound radiation is used to exert a low-frequency (in $\mathrm{kHz}$ range) force on a rigid magnetized target immersed in a viscoelastic medium. In response, the target vibrates in a pattern determined by viscoelastic properties of medium. We report the relation between the magnetic field signal and the incident ultrasonic pressure field in terms of the mechanical parameters of the medium. Simulations were conducted to demonstrate a simple approach based on using amplitudemodulated ultrasound to generate a dynamic acoustic radiation force on a magnetic target. The magnetic field generated by vibration of this target is then obtained and used to estimate the radiation-force-induced displacement as a function of time. It was observed that the intensity of the dynamic component of the magnetic field caused by the acoustic excitation is high enough to be registered by a conventional magnetic sensor. This proposed methodology presents a powerful tool for evaluation of acoustic radiation force as well as the mechanical properties of soft material.

\section{9:50-10:20 Break}

\section{0:20}

2aBB8. Shear wave dispersion ultrasound vibrometry of gel dosimetry phantoms. Silvio Vieira (Dept. de Fis. e Mat. da FFCLRP, Univ. de São Paulo, Av. Bandeirantes, 3900-Bairro Monte Alegre-Ribeiräo Preto-SP, Ribeiräo Preto, SP 14040-901, Brazil, silvio@pg.ffclrp.usp.br), Matthew Urban (Mayo Clinic, Rochester, MN 55901), Andre Baggio (Univ. de São Paulo, Ribeiräo Preto, SP 14040-901, Brazil), Antonio Carneiro (Univ. de So Paulo, Ribeiräo Preto, SP 14040-901, Brazil), and Mostafa Fatemi (Mayo Clinic, Rochester, MN 55901)

Shear wave dispersion ultrasound vibrometry (SDUV) has been introduced to use the dispersive nature of shear wave speed to locally estimate the material properties or tissue. Shear waves were created using a mechanical shaker and pulsed radiation force. Using the information from the phase one can estimate the shear velocity. A theoretical Voigt model allows us to find, at different frequencies $(100-400 \mathrm{~Hz})$, the viscosity and shear elasticity of the polymer gel dosimeter. The aim of this work was to perform the SDUV and mechanical vibrometry techniques to study the viscoelastic properties of absorbed dose phantoms. The experiments were performed using five phantoms of different absorbed doses varying 10 up to $50 \mathrm{~Gy}$, while a nonirradiated phantom was kept as a control. The SDUV technique was performed using a pulsed radiation force to vibrate the gel provide by a confocal transducer with a focal depth of $70 \mathrm{~mm}$, nominal frequency of $3 \mathrm{MHz}$. For the mechanical vibrometry and SDUV experiments, shear wave motion measurements were made with a commercial ultrasound machine with a research using a $10 \mathrm{MHz}$ linear array transducer. The quantitative elasticity and viscosity results for these two experiments were compared.

\section{$10: 35$}

2aBB9. Targeted ultrasound contrast agents-Mediated endothelial permeability. Pavlos Anastasiadis (Dept. of Mech. Eng., Univ. of Hawaii at Manoa, 2540 Dole St., Honolulu, HI 96822, pavlos@hawaii.edu), Chris N. Layman, and John S. Allen (Univ. of Hawaii at Manoa, Honolulu, HI 96822)

Endothelial cell dysfunction is considered to play a major role in the early stages of atherosclerosis. Targeted ultrasound contrast agents offer potential for therapeutic treatment though the mechanisms for enhanced ultrasound and microbubble mediated transport across endothelial layers are not well understood. In this study, we evaluate the endothelial cell-to-cell and cell-to-substrate gaps with the electric cell-substrate impedance sensing system (ECIS, Applied BioPhysics, Troy, NY). ECIS can detect the nanometer order changes of cell-to-cell and cell-to-substrate distances separately. Targeted ultrasound contrast agents (UTC) were employed in conjunction with the application of ultrasound, while continuous measurements of electrical resistance across the endothelial monolayers were conducted in real-time for the determination of permeability. The measurements were performed in both, the presence and absence of UCA. Additional complementary, noninvasive acoustic measurements of local changes in the mechanical properties of the endothelial monolayers were conducted at high-frequency $(100 \mathrm{MHz})$ with a time-resolved scanning acoustic microscope. [This work was supported by the National Institutes of Health Grants NIH 2 P20 RR01645305A1 and NIH 2 G12 RR0030161-21.] 
10:50

2aBB10. Targeted ultrasound contrast agents for the imaging of biofilm infections. Pavlos Anastasiadis (Dept. of Mech. Eng., Univ. of Hawaii at Manoa, 2540 Dole St., Honolulu, HI 96822, pavlos@hawaii.edu), Kristina Mojica, Michelle L. Matter, and John S. Allen (Univ. of Hawaii at Manoa, Honolulu, HI 96822)

Targeted ultrasound contrast agents (UCA) offer a novel, potential method for diagnostic imaging of biofilm infections. Currently, there is no established method for molecular imaging of in vitro biofilm infections with any type of modality. For infective endocarditis, the case of biofilm formation on damaged or diseased heart valves, an early diagnostic method might greatly reduce the associated high mortality rate. Fluorescently labeled lectins were used with targeted ultrasound contrast agents in conjunction with epifluorescence microscopy and time-resolved scanning acoustic microscopy for visualization and characterization of the extracellular polymeric substances (EPS) of two types of infectious biofilms (Staphylococcus aureus and Pseudomonas aeruginosa). Noninvasive acoustic measurements of the biofilms were conducted at high-frequency $(100 \mathrm{MHz})$ with a time-resolved scanning acoustic microscope. Measurements of the reflection and transmission coefficients, over a broad frequency spectrum for a range of biofilm growth stages in both, the presence and absence of targeted UCA were obtained. The biofilm morphology and structure were also investigated with acoustic microscopy as a function of growth. [This work was supported by the National Institutes of Health Grants NIH 2 P20 RR016453-05A1 and NIH 2 G12 RR0030161-21.]

\section{1:05}

2aBB11. Transcutaneous acoustic palpation (TAP) for the localization of peripheral, painful tissue. Pierre D. Mourad (Appl. Phys. Lab., Dept. of Neurosurgery, Box 356470, Univ. of Washington, Seattle, WA 98195 , pierre@apl.washington.edu), Michel Kliot, and Jeffrey G. Jarvik (Univ. of Washington, Seattle, WA 98195)

Localizing painful tissue (pain generators) is central to medicine. Here we sought to demonstrate that intense focused ultrasound (iFU) can successfully differentiate normal from painful tissue. Rats were injected with complete Freunds adjuvant in a hind paw to create an inflammatory injury, or underwent a surgical procedure that damaged the nerve enervating the paw, creating a neuropathic injury. Each created a paw sensitive to external stimulation relative to the contralateral paw. iFU was applied individually to each hind paw in increasing doses until the animal withdrew either paw consistently from the iFU stimulus, thereby defining the iFU threshold dose for that animal. This data was correlated with paw withdrawal latencies to a heat lamp (Hargreaves) test. Sensitized paws responded to lower intensities and doses of iFU than control paws greater than 95 percent of the time with sensitivities and specificities generally greater than 90 percent. In general, iFU threshold tests and Hargreaves tests did not affect one another, a functional test of the safety of iFU. This preliminary evidence supports the hypothesis that iFU can safely discriminate between painful and normal tissue. Future work will use image-guided iFU to localize deep rather than superficial pain generators.

\section{1:20}

2aBB12. A stabilized forward elasticity finite element formulation yields a stable and convergent inverse elasticity solution. Carlos E. Rivas, Paul E. Barbone (Mech. Eng., Boston Univ., 110 Cummington St., Boston, MA 02215, barbone@bu.edu), and Assad A. Oberai (Rensselaer Polytech. Inst., Troy, NY 12180)

"Elastography" refers to the procedure of using ultrasound to image tissue deformation. Images showing tissue deformation are sensitive to distributions of tissue mechanical properties. The mechanical properties themselves may be quantified by solving an inverse elasticity problem. An iterative approach to solving the inverse problem can be formulated by repeated solutions of the forward problem. That is, the shear modulus distribution sought is that which predicts a displacement field most consistent with the measured displacement field. Here we show that given plane displacement measurements for plane stress elasticity, the continuous elastic equilibrium equation uniquely determines the modulus distribution. On the other hand, we shall also demonstrate that the discrete elasticity equations from standard FEM discretization does not, even at the limit of infinite mesh refinement. We diagnose the problem as an underenforcement of the elasticity equations. With this knowledge, we have been able to design new forward elasticity FEM formulations that provide provably convergent forward and inverse elasticity solutions.

\section{1:35}

2aBB13. Ultrasonic properties of normal and cancerous human breast cell lines. Sang-Mo Shin (School of Medical System Eng., Gwangju Inst. of Sci. and Technol., 1 Oryong-dong, Buk-ku, Gwangju 500-712, Korea, sshin@gist.ac.kr), Koeng-Mo Sung, Hyun-Woo Koh (Seoul Natl. Univ., Seoul 151-600, Korea), and Pavlos Anastasiadis (Univ. of Hawaii at Manoa, Honolulu, HI 96822)

Time-resolved scanning acoustic microscopy (SAM) equipped with time-resolved signal analysis offers unique possibilities for studying the mechanical properties of cells in vitro. The method is nondestructive and noninvasive on a cellular level, which enables real-time measurements on living cells. The ultrasonic attenuation coefficients and sound speeds of MCF 10A normal breast cells and MCF 7 weakly invasive cancerous cells were measured and compared using a time-resolved SAM operating at $0.86 \mathrm{GHz}$ center frequency and with $5 \mathrm{~ns}$ pulse duration. The attenuation coefficients of MCF 10A and MCF 7 cells were found to be $126 \pm 67$ and $443 \pm 129 \mathrm{~dB}$, respectively, while the sound speeds of the same cells were $1583 \pm 24$ and $1736 \pm 61 \mathrm{~m} / \mathrm{s}$, respectively. Both the attenuation coefficient and sound speed of MCF 7 cells were higher than those of MCF 10A cells. In particular, the difference in the attenuation coefficients was pronounced. The relative differences in sound speed and attenuation coefficient could be utilized to differentiate normal cells from cancerous cells. [Weiss et al. IEEE Trans. Ultrasonics, Ferroeletrics, and Freq. Contrl. 54, 2257-2271 (2007)] [Work supported by Korean Science and Engineering Foundation.] 


\title{
Session 2aEA
}

\section{Engineering Acoustics and Physical Acoustics: Lasers in Underwater Acoustics}

\author{
Kenneth M. Walsh, Chair \\ K M Engineering Ltd., 51 Bayberry Ln., Middletown, RI 02842 \\ Chair's Introduction-7:55 \\ Invited Papers
}

8:00

Scanning laser vibrometers have been in use for many years in acoustic measurements. Unique methods of characterizing both active and passive devices or structures with vibrational modes have been developed by NUWC personnel; these are combinations of signal generation techniques and physics-based data analysis tools. The signal generation techniques reduce the time for potentially lengthy scans at multiple frequencies through the use of a "comb" waveform, [J. Acoust. Soc. Am. 116(5), (2004)], if the device under test is known to be linear. Scans can be done in either air or water. An analysis technique using the Helmholtz integral (using NUWC's MATLAB-based CHIEF code; Schenck and Benthein, NOSC 1970, 1988) allows analysis from an in-air scan of a transducer face (before encapsulation) to predict the transducer's far-field beam patterns under some conditions. This is especially valuable as a timesaving tool to ensure that the transducer is on track to achieve the final design intent. Examples of the techniques applied to various transducer designs will be presented; for comparison, single-tone results also will be shown.

\section{8:25}

2aEA2. On continuous-line acoustic array behavior in a laser Doppler sound pressure measurement system. Michael S. McBeth (Space and Naval Warfare Systems Ctr. Atlantic, 204 Indian Springs Rd., Williamsburg, VA 23185, m.s.mcbeth@ieee.org) and Yongxiang Hu (NASA Langley Res. Ctr., Hampton, VA 23681)

Continuous-line acoustic array behavior in a laser doppler anemometry (LDA) based sound pressure measurement system is considered. Traditional LDA ultrasound measurement systems intentionally minimize the laser beam intersection or interference fringe volume to better achieve point measurements of the sound field. The large laser beam diameters due to beam spreading in remote sensing applications from space or airborne platforms make this approach impractical. Since the sound pressure signal is integrated over the interference fringe volume, continuous-line acoustic array behavior is expected to be observed proportional to the length of the laser light path through the interference fringe volume. This behavior is analyzed from a theoretical perspective and an experimental setup to test for continuous-line acoustic array behavior in a LDA sound pressure measurement system is presented.

\section{8:50}

2aEA3. Measurement and imaging of high-frequency sonar fields using acousto-optic tomography. Pete Theobald, Stephen Robinson, Triantafillos Koukoulas, and Gary Hayman (Natl. Physical Lab., Hampton Rd., Teddington, Middlesex TW11 OLW, UK, pdt@npl.co.uk)

The acoustic near-field of a high-frequency sonar array transducer has been measured using a laser scanning acousto-optic method and reconstructed using a tomographic technique similar to that used for x-ray computed tomography. This measurement, using a laser interferometer to scan a plane in front of the transducer for a series of transducer rotations, provides an image of the acoustic field for a plane in front of the transducer array. This method allows measurement of the acoustic field without perturbing the field being measured, which can occur when using the planar hydrophone scanning method, provides high spatial resolution with minimal phase averaging across the measurement aperture and provides potential for significantly decreasing the scan time. Measurements have been performed on two 1-3 composite transducer arrays at frequencies between 330 and $500 \mathrm{kHz}$. These are compared with planar hydrophone scans obtained using a $1.5-\mathrm{mm}$ probe hydrophone. Both parallel and fan beam tomographic scan geometries have been considered along with the effect of lateral scan resolution versus angular rotation resolution.

$$
\text { 9:15 }
$$

2aEA4. Laser light scattering by bubbles in water: Fundamentals and applications to acoustics. Philip L. Marston (Phys. and Astron. Dept., Washington State Univ., Pullman, WA 99164-2814, marston@wsu.edu)

Laser light scattering is sometimes used as an alternative to acoustical methods for monitoring bubbles in seawater. There has also been interest in using lasers to investigate bubbles in wakes. In some cases light scattering by bubbles has been used in conjunction with acoustical measurements to characterize dynamics of bubbles radiating sound [J. S. Stroud and P. L. Marston, J. Acoust. Soc. Am. 94, 2788-2792 (1993)]. In applications such as these it is important to understand the optical properties of bubbles that differ significantly from drops and particles. Examples include critical angle scattering and the transition to total reflection [D. S. Langley and P. L. Mar- 
ston, Appl. Opt. 23, 1044-1054 (1984)], forward scattering and extinction [D. S. Langley and P. L. Marston, Appl. Opt. 30, 3452-3458 (1991); J. S. Stroud and P. L. Marston, cited previously], glory back-scattering enhancements and shape effects [W. P. Arnott and P. L. Marston, J. Opt. Soc. Am. A5, 496-506 (1988); Appl. Opt. 30, 3429-3442 (1991)] and Brewster angle scattering. Some optical effects of coatings on bubbles (which can occur naturally) have also been modeled [P. L. Marston, Appl. Opt. 30, 3479-3484 (1991)]. [Research supported by ONR between 1980 and 1995.]

\section{9:40}

2aEA5. A laser-based method of detecting underwater sound through an ice layer. Lynn T. Antonelli and Fletcher A. Blackmon (Naval Undersea Warfare Ctr., Code 1512, 1176 Howell St., Newport, RI 02841, lynn.antonelli@navy.mil)

An aerial, remote sensor has been devised to detect underwater sound through an ice layer by optically measuring the resulting vibration velocity on the ice surface, using a laser Doppler vibrometer. The laser was focused on an ice block approximately 8 in. wide by $12 \mathrm{in.} \mathrm{long} \mathrm{and} 3.5 \mathrm{in}$. thick floating in a tank of water $16 \mathrm{in}$. deep. A submerged transducer was used to generate underwater acoustic signals to provide ice surface vibrations for the sensor to detect. Although acoustic attenuation through the ice was not an issue for this test, laser alignment was crucial. Since sensor reliability depends on receiving laser reflections from the ice surface, the laser was steered nearly perpendicularly onto the ice surface that had both smooth and rough features. The broadband optical sensor provides a remote sensing capability for measuring ice surface vibrations in response to underwater acoustic signals such as from marine mammals, passive or active sonar, and from underwater acoustic communication systems. The results of this landmark experiment will be presented, which demonstrate the feasibility for detecting underwater sound through an ice layer by probing the ice surface with a laser vibrometer, paving the way for in-air sensing in arctic regions.

$$
\text { 10:05-10:20 Break }
$$

\section{0:20}

2aEA6. Remote, aerial, translayer, nonlinear downlink underwater acoustic communication. Fletcher A. Blackmon (Naval Undersea Warfare Ctr., Code 1512, 1176 Howell St., Newport, RI 02841, fletcher.blackmon@navy.mil) and Lynn T. Antonelli (Naval Undersea Warfare Ctr., Newport, RI 02841)

The nonlinear mechanism for optical to acoustic energy conversion is explored for optoacoustic communication from an in-air platform to a submerged platform. This downlink communication can provide a bell ringer function and transmission of text and data. The nonlinear conversion mechanism, also known as the nonlinear optoacoustic regime where focused laser energy is converted to sound at the air-water interface, involves a phase change of the water medium through evaporation and vaporization leading to plasma production. The nonlinear conversion mechanism provides a more efficient, i.e., higher source level, controllable method for producing underwater acoustic signals. A means of deterministically controlling the spectrum of the underwater acoustic signal has been investigated and demonstrated by varying the laser-pulse repetition rate to provide $M$-ary frequency shift keyed signaling. This physics-based conversion process provides a methodology for providing low probability of intercept signals whose information is embedded in noiselike signals. These laser generated signals can then be used in a frequency hopped spread spectrum technique with the use of the proper receiver structures to take advantage of the frequency diversity and periodicity inherent in this type of signal structure that could also be used to combat frequency selective fading in underwater acoustic channels.

\section{0:45}

2aEA7. Intense underwater laser acoustic source for Navy applications. Theodore G. Jones, Melissa K. Hornstein, Antonio C. Ting (U.S. Naval Res. Lab., Plasma Phys. Div., 4555 Overlook Ave. SW, Washington, DC 20375, ted.jones@nrl.navy.mil), and Zachary W. Wilkes (Res. Support Instruments, Inc., Lanham, MD 20706)

An intense remote underwater laser acoustic source is under development at the Naval Research Laboratory. In a novel configuration, a tailored intense laser pulse can be designed to propagate many meters underwater and compress at a predetermined remote location. Controlled compression of these optical pulses is governed by a combination of optical group velocity dispersion and nonlinear Kerr self-focusing. Optical compression can result in laser-induced breakdown, localized heating, and acoustic shock generation. Recent experiments include near-field acoustic source characterization using lens-focused 400 and $800 \mathrm{~nm}$ pulses of a Ti:sapphire laser, as well as 532 and $1064 \mathrm{~nm}$ pulses of a YAG laser. Sound pressure levels over $210 \mathrm{~dB}$ were achieved using a compact laser. Acoustic source characterization includes measurements of photoacoustic energy conversion efficiency, acoustic power spectrum, and directivity. Nonlinear optical studies included the precise measurement of the Kerr index of water at 400 and $800 \mathrm{~nm}$, as well as conditions for optical filament generation, and their effects on acoustic signals. Planned experiments include tests in a bubbly salt water tank. Experimental results will be presented, and laser sources and techniques for underwater acoustic generation will be compared. [This work is supported by the U.S. Office of Naval Research.] 


\title{
Contributed Papers
}

11:10

2aEA8. Transducer characterization by laser Doppler vibrometry. Martin P. Cooling, Victor F. Humphrey (Inst. Sound \& Vib. Res., Univ. of Southampton, Southampton, SO17 1BJ, UK, mc@isvr.soton.ac.uk), Pete D. Theobald, and Stephen P. Robinson (Natl. Physical Lab., Teddington, Middlesex, TW11 0LW, UK)

A laser Doppler vibrometer (LDV) can be set up such that its laser beam passes through the glass wall of a water tank to be incident upon the radiating surface of an immersed ultrasonic transducer. Scanning the laser beam across a transducer in this way has the potential to be a fast, noninvasive method for source characterization and, in turn, field prediction. Such measurements are, however, significantly complicated by the acousto-optic interaction-that is, the effect on the measurements of the acoustic field through which the laser beam passes. To increase understanding of the resultant acousto-optic artifact, a detailed simulation of the LDV measurement of a circular, plane-piston transducer emitting a tone-burst has been created The use of a transient field is important for simulation and experiment, such that measurements can be made over a time window which ends before any acoustic signal reaches the water tank boundaries. The simulation results show a significant acousto-optic artifact contribution to the surface velocity data, but also that for some applications useful field predictions may be made in spite of this. To complement the simulations, experimental measurements have been made using a commercial LDV (Polytec PSV-400) on a $500 \mathrm{kHz}$ circular transducer.

\section{1:25}

2aEA9. Measurement of the resonance frequency of a bubble using a laser Doppler vibrometer. Theodore F. Argo, IV and Preston S. Wilson (Mech. Eng. Dept. and Appl. Resource Labs., The Univ. of Texas at Austin, Austin, TX 78712-0292)

Knowledge of the behavior of bubbles that are confined within tubes or channels has a direct impact on the development of various medical applications and industrial processes. In such confined spaces, bubbles are not easily observed by traditional experimental means, such as acoustically with hydrophones, or optically with Mie scattering or stroboscopy. Further, facilitating observation with these methods may significantly perturb the system. A laser Doppler vibrometer (LDV) requires only a narrow $(\sim 1 \mathrm{~mm}$ diam) line-of-sight access for the beam and illumination of the bubble does not perturb its dynamics. LDV measurements of the resonance frequency and quality factor of an air bubble suspended in a small water tank are presented to demonstrate the utility of this technique. The measurements are compared to a model that includes the acoustic effect of the tank walls. The precision of the technique was found to be similar to the precision of a traditional hydrophone-based acoustic technique.

TUESDAY MORNING, 19 MAY 2009

EXECUTIVE SALON II/III, 10:00 A.M. TO 12:00 NOON

\section{Session 2aED}

\section{Education in Acoustics: Hands-On Experiments for High School Students}

\author{
Uwe J. Hansen, Chair \\ Indiana State Univ., Dept. of Chemistry and Physics, Terre Haute, IN 47809
}

Chair's Introduction-10:00

\begin{abstract}
Approximately 20 acoustics experiments will be set up, ranging in complexity from simple resonance on a string to ultrasonic levitation. Around 40 local high school science students will perform the experiments with help from ASA scientists and students. Regular ASA conference participants are welcome to the session as long as they do not interfere with student experimentation.
\end{abstract}




\title{
Session 2aMU
}

\section{Musical Acoustics: Wind Instruments I}

\author{
Thomas D. Rossing, Chair \\ Stanford Univ., CCRMA, Stanford, CA 94305
}

Invited Papers

\begin{abstract}
8:00
2aMU1. Three decades of wind instrument research at Brigham Young Univ. William Strong (Dept. of Phys. and Astron., Brigham Young Univ., Provo, UT 84602)

Wind instrument research at Brigham Young University during the 1970s, 1980s, and 1990s will be reviewed. In more or less chronological order, the research was as follows: (1) Calculation of input impedances of an oboe; (2) predictor coefficient analysis /synthesis of French horn tones; (3) functional model of a simplified clarinet; (4) calculated and measured modal frequencies of brass instruments; (5) calculation and measurement of flute impedances and standing waves; (6) calculation and measurement of horn impedances; (7) simulation of a player-clarinet system; (8) optimization of clarinet toneholes; (9) simulation of a player-trumpet system; (10) stroboscopic measurement of the lip motions of a trombone player; (11) calculation and measurement of nonlinear effects in loud trombone tones; (12) finite element modeling of the lips of a trombone player. [Work supported by the Institute for Research in Musical Acoustics.]
\end{abstract}

\section{8:30}

2aMU2. Spectral component relationships in wind instrument transients. Peter L. Hoekje (Dept. of Phys. and Astron., BaldwinWallace College, 275 Eastland Rd., Berea, OH 44017, phoekje@bw.edu)

Playing characteristics of a wind musical instrument are related to its transient response, the efficiency of its sound production, and the stability of its sustained tones, as determined partly by the acoustical resonances of the instrument, and their nearly harmonic relationships. These can be explored using analysis of instrument attack transients by tracking development of the spectral components. A coupled oscillator model is used to explain the benefits to an instrument's response due to resonances at harmonics of the playing frequency. Furthermore, the slight deviation from harmonic relationships of an instrument's resonances leads to a shift in its tone center during a crescendo. A resonance at a frequency above the tone-hole lattice cutoff can affect the playing behavior of flutes and cornettos. The player's airway in reed woodwinds and brasses is as much a part of the acoustic system as the instrument proper, and its resonances can have a correspondingly important effect.

\section{Contributed Papers}

\section{9:00}

2aMU3. Impulse response reverb as a tool for voicing organs. Michael Steppat (Erlenweg 17, D-14558 Nuthetal OT Saarmund)

The sound of a flue pipe can be adjusted by voicing. With parameters like the cut-up of the upper lip and their width, the opening in the foot, the height of the languid, and the width of the slit the voicer can influence the promptness of speech and adjust the intensity of the starting transient. The acoustical environment (especially the reverberation time) plays an important role in the reception of the starting transient, therefore the organ builder has to also take the architecture into account. Convolution reverb allows a simulation of the reverb at different positions. Sampled organ sounds with modified starting transients or modeled sounds can be played on a computer and mixed with a convolution reverb using measured impulse responses from different positions of the room. This computer model can be used during the design process to estimate the parameters for the voicing. Results of measurements in a church building and different modeling techniques will be presented

9:15

2aMU4. Measuring the Young's modulus of reeds from American reed organs. Michael B. Wilson (Juniata College, 1700 Moore St., Huntingdon, PA 16652,wilsomb05@juniata.edu), Alexander M. Ramm, and James P. Cottingham (Coe College, Cedar Rapids, IA 52402)

A method of wave coefficients has been used to measure the Young's modulus of brass reeds from American reed organs. Although for some pur- poses these reeds can be approximated as uniform cantilever beams, their cross sections are sufficiently nonuniform that simple methods such as measuring beam displacement for a known applied force or using resonant frequencies of transverse and torsional modes of vibration are not sufficient to obtain accurate results. In the wave coefficient method [Y. Liao and V. Wells, J. Sound Vibr. 295, 165-193 (2006)] a wave number is found that forces data at all measurement points to conform to the general forcedvibration solution for a beam, implying that they have the same wave coefficients. This method also assumes a uniform cross section but does not require measurements along the entire length of the beam and was applied only to the most uniform sections of the reeds. Three reeds of the same frequency were selected, with cross sections of varying uniformity. The resulting measurements of the Young's modulus seemed consistent with expected values for brass. For one reed with nearly uniform cross section the wave coefficient method was in good agreement with the simpler methods.

$$
\text { 9:30 }
$$

2aMU5. The influence of curvature on the vibration and tone quality of pipe organ reed tongues. G. R. Plitnik (Dept. of Phys., Frostburg State Univ., Frostburg, MD 21532) and J. Angster (Fraunhofer Inst. fur Bauphysik, Stuttgart, Germany)

Given certain design constraints, such as the type of stop being voiced and the desired tone quality, organ reed voicers must curve each tongue so as to produce the best and most stable tone, as well as maintaining a consistent tone quality across an entire rank of pipes. This study investigated the effects of varying tongue curvatures on two pipes, an F2 Trompette and an 
F2 Clarinet. Reed vibration was measured under typical conditions by laser vibrometer; the pressure waves in the boot and shallot were measured by means of one-eighth inch microphones and the emitted sound was recorded at the egress. By integrating the velocity data, the displacement of the reed may be plotted in tandem with the pressure difference across the reed, allowing tongue position to be plotted versus the pressure variation during one steady-state cycle for each reed while vibrating. These graphs can then be compared and correlated to the observed tone quality for each of the differently curved reeds.

\section{9:45}

2aMU6. Acoustics of single-reed duck calls. Nicholas P. Goodweiler (College of Eng., Univ. of Iowa, Iowa City, IA 52242, nicholas-goodweiler@uiowa.edu) and James P. Cottingham (Coe College, Cedar Rapids, IA 52402)
A single-reed duck call features a reed of Mylar or similar elastic material mounted above a curved tone channel. The arrangement resembles that of a clarinet, except that the extreme curve of the tone channel (compared to the relatively flat lay of the clarinet) allows for a rather large volume airflow. In addition, the flexibility of the reed results in a low fundamental frequency (typically $100-140 \mathrm{~Hz}$ ) so that the pitch of the call is close to the natural frequency of the reed, modified only slightly by the short resonating barrel. As the blowing pressure is increased, there is a gradual rise in pitch, punctuated by periodic jumps from one apparent regime of vibration to another. As the blowing pressure is lowered, some hysteresis effects are observed. Several calls built by different makers have been studied, each with a differently proportioned barrel and tone channel. Some of the phenomena observed include reed velocity and displacement, changes in effective reed length as a function of blowing pressure, and effects of barrel design on tone quality. Some observations have also been made of the volume airflow and effects of attaching pipe resonators.

TUESDAY MORNING, 19 MAY 2009

MAIN AUDITORIUM, PORTLAND BUILDING, 8:00 A.M. TO 5:00 P.M.

\section{Session 2aNS}

\section{Urban Design with Soundscape in Mind}

\section{A Symposium on Urban Planning with the Consideration of Noise Impacts and the People Concerned}

\section{8:15}

Introduction to Symposium and Keynote Speaker

Kerrie Standlee. P.E., Daly-Standlee \& Associates, Inc., Beaverton, OR

\section{8:20}

The Political Need to Consider Noise Impacts on Urban Livability City of Portland Government Official

\section{$8: 45$}

The Physical Need to Consider Noise Impacts on Urban Livability Kerrie Standlee. P.E., Daly-Standlee \& Associates, Inc., Beaverton, OR

\section{9:10}

The Historic Approach in Addressing Noise Impacts on Urban Livability

9:15

Noise Ordinances and Zoning Laws Paul Herman, Retired, Noise Control Officer, City of Portland, Portland, OR Paul Van Orden, City of Portland Noise Control Officer, Portland, OR Jerry Wilson, Retired, Oregon DEQ Environmental Noise Anaylst Ioana Park, P.E., BRC Acoustics, Seattle, WA

10:15 - 10:30 Break

Use of Building Site and Design to Address Noise Impacts Bennett Brooks, P.E., Brooks Acoustics Corporation, Vernon, CT Daniel C. Bruck, Ph.D., BRC Acoustics, Seattle, WA 
$12: 10-1: 30$ Lunch

$1: 30$

A New Approach in Addressing Noise Impacts in an Urban Setting-The need to consider people's minds

\section{$1: 35$}

Soundscape in the Urban Environment - What does it mean

Brigitte Schulte-Fortkamp, Technische Univ. Berlin, Inst. of Fluid Mechanics and Engineering, Berlin, Germany

Bennett Brooks, P.E., Brooks Acoustics Corporation, Vernon, CT

$$
\text { 2:35 }
$$

Application of Soundscape Techniques

Gary Siebein, School of Architecture, University of Florida, Gainesville, FL

3:35

Closing Remarks

Kerrie Standlee. P.E., Daly-Standlee \& Associates, Inc., Beaverton, OR

3:50

Tour of Portland Eastside Esplanade for Hands-On Demonstration of Soundscape Techniques

Tour led by Soundscape Presenters

5:00

Symposium Adjourned

TUESDAY MORNING, 19 MAY 2009

BROADWAY III/IV, 8:00 TO 10:15 A.M.

\title{
Session 2aPAa
}

\section{Physical Acoustics: Cavitation: Mechanisms and Physical Effects}

\author{
Thomas J. Matula, Chair \\ Applied Physics Lab., Univ. of Washington, Seattle, WA 98105-6698
}

\section{Contributed Papers}

\section{8:00}

2aPAa1. Laser-induced dielectric breakdown as a method for noncontact pressure measurement. Jonathan R. Sukovich, Todd W. Murray, and R. Glynn Holt (Dept. of Mech. Eng., Boston Univ., 110 Cummington St., Boston, MA 02215)

Being able to accurately measure pressure fields in acoustic experimentation is of critical importance for understanding and describing observed acoustical phenomena. However, in many experimental situations, it is undesirable or impossible to measure the acoustic field using a transducer in contact with the acoustic host medium. It becomes useful then to have alternative, noncontact, methods for reliably and accurately measuring pressure fields in experiments. One alternative to traditionally invasive means, such as the use of a hydrophone, to measure pressure, is to use focused, short (5-7 ns), high power laser pulses to cause dielectric breakdown. Breakdown events can be detected by looking for the signature, near instantaneous, flash of broadband light generated by the subsequent ionization cascade. This procedure can then be repeated under different known hydrostatic pressures to establish pressure's effect on the breakdown strength of water. Once a relationship is established, the process can be used to map local pressures in acoustic fields of sufficiently long period as to be considered hydrostatic in relation to the laser pulse duration. [Work supported by the US Army Space and Missile Command.]

\section{$8: 15$}

2aPAa2. Acoustic measurement of bubble size and position in an ink jet printhead. Arjan van der Bos, Roger Jeurissen, Michel Versluis, Detlef Lohse (Phys. of Fluids, Dept. of Appl. Phys., Univ. of Twente, P.O. Box 217, 7500 AE, Enschede, The Netherlands), Hans Reinten, Herman Wijshoff, Marc van den Berg, and Jos de Jong (Oce Technologies B.V., 5900 MA, Venlo, The Netherlands) 
A bubble can be entrained in the ink channel of a piezo-driven ink jet printhead leading to malfunction. Here, an acoustic sizing method for the volume and position of the bubble is presented. The influence of the bubble on the channel acoustics is detected by the piezo-actuator operating in a sensing mode. The acoustic response is also modeled in a linearized bubble dynamics model and it is demonstrated how the size of the bubble and its position within the channel can be inferred from the inverse problem. The validity of the presented method is supported by time-resolved optical observations of the bubble dynamics visualized in a glass connection channel. The combined optical and acoustical recordings are compared to the model, where a very good agreement is found. [This work is part of the research program of the Technology Foundation STW and financially supported by Océ Technologies B.V.]

\section{8:30}

2aPAa3. Laser nucleation of bubble clusters in a spherical resonator Phillip A. Anderson, Todd W. Murray, and R. Glynn Holt (Dept. of Mech. Eng., Boston Univ., 110 Cummington St., Boston, MA 02215)

Due to the strong energy focusing found in sonoluminescence, there is much interest in high-energy bubble collapses. There is evidence in the literature that the collective collapse of a bubble cluster is more violent than a single bubble collapse under similar conditions. Precise control over the spatial and temporal placement of bubbles in an acoustic field would seem critical for achieving the strongest collapses, but nucleating bubbles acoustically is not precise or repeatable and does not allow for the creation of arrays of bubbles. A method is presented for creating two- and three-dimensional bubble arrays in a controllable way. A pulsed Nd:YAG laser is used with custom diffraction gratings and focusing optics to nucleate bubble arrays in a spherical resonator at high static pressure. A specific implementation will be presented, along with results addressing uniformity and repeatability. [Work supported by the US Army Space and Missile Command.]

\section{$8: 45$}

2aPAa4. Collapse dynamics of laser-nucleated bubble clusters in a spherical resonator. Phillip A. Anderson, Todd W. Murray, and R. Glynn Holt (Dept. of Mech. Eng., Boston Univ., 110 Cummington St., Boston, MA 02215)

The collective collapse of bubble clouds or clusters is important to many applications, including underwater sound propagation, shock-wave lithotripsy, and focused ultrasound therapy. Energetic collective cluster collapses appear to happen only when there is sufficient nuclei density. In order to study this phenomenon, we have implemented a laser-based method for creating clusters consisting of planar or cubic arrays of bubbles with controllable interbubble spacing. The growth and collapse of the arrays are observed using ultrahigh-speed photography and other diagnostics. Effects of interbubble spacing and nucleation phase with respect to the acoustic period are studied. [Work supported by the US Army Space and Missile Command.]

\section{9:00}

2aPAa5. Detecting cavitation in liquid mercury exposed to a high-energy pulsed proton beam. Nicholas J. Manzi, Robin O. Cleveland, R. Glynn Holt, and Ronald A. Roy (Dept. of Mech. Eng., Boston Univ., Boston, MA 02115)

The Oak Ridge National Laboratory spallation neutron source (SNS) employs a high-energy proton beam incident on a liquid mercury target to generate short bursts of neutrons. Concomitant with neutron production is the rapid heating of the mercury, resulting in the formation of acoustic shock waves and the nucleation of cavitation bubbles. The subsequent collapse of these cavitation bubbles may result in the eventual erosion of the target's steel walls. We report on preliminary measurements using two passive cavitation detectors (megahertz frequency focused and unfocused PZT transducers), installed in a mercury test target to monitor cavitation activity for proton beam energies ranging from $0.041 \mu \mathrm{C}$ to $4.1 \mu \mathrm{C}$. Cavitation was initially detected for a beam "charge" of $0.205 \mu \mathrm{C}$ by the presence of an acoustic emission approximately $120 \mu$ s after arrival of the incident beam, which is characteristic of inertial cavitation collapse. As beam energy was increased, the collapse time increased until the lifetime of the bubbles exceeded the reverberation time of the chamber $(\sim 300 \mu \mathrm{s})$ at which point complex emissions signatures were detected due to the interaction of the reverberation signal and the cavitation bubbles.

\section{9:15}

2aPAa6. Production of cavitation erosion damage by means of an electromagnetic shock wave source. Qi Wang, Nicholas J. Manzi, R. Glynn Holt, Ronald A. Roy, and Robin O. Cleveland (Dept. of Mech. Eng., Boston Univ., 110 Cummington St., Boston, MA 02215, robinc@bu.edu)

Central to a spallation neutron source (SNS) is a vessel of liquid mercury. During SNS operation acoustic pulses, with pressures in excess of $10 \mathrm{MPa}$, are generated in the mercury likely resulting in cavitation, which may erode the walls. We developed an electromagnetic shock wave source to generate similar pressures and cavitation. An electromagnetic source consists of a coil placed close to a metal plate, which is in contact with a fluid. The source is driven by discharging a capacitor through the coil, which results in a repulsive force on the plate launching acoustic waves in the fluid. A $75 \mathrm{~mm}$ diameter source coupled to water was fabricated, and the plate motion was measured by laser Doppler vibrometry and the acoustic field by a piezoelectric needle for different capacitance, charging voltage, number of coils, and plate properties. For a $2 \mu F$ capacitor charged to $12 \mathrm{kV}$ and discharged through a 28-turn coil with a $0.8 \mathrm{~mm}$ thick aluminum plate (spherically focused) the peak pressure measured in water was $20 \mathrm{MPa}$. Cavitation clouds were produced over a wide range of voltages (imaged with $15 \mu$ s exposure) with lifetimes around $300 \mu$ s and produced erosion craters on a porous solid. [Work supported by ORNL-SNS, UT-Battelle, USDOE Contract DE-AC05-00OR22725.]

\section{9:30}

2aPAa7. The ultreo: A novel toothbrush that combines sonic and ultrasound physics. Pierre D. Mourad (Appl. Phys. Lab. and Dept. of Neurosurgery, Box 356470, Univ. of Washington, Seattle, WA 98195, pierre@apl.washington.edu), Frank Roberts (Dept. of Peridontics, Univ. of Washington, Seattle, WA 98195), and Chris McInnes (Ultreo, Inc.)

Efficacious, daily oral health care represents an important part of maintaining the overall health of individuals. Here we sought to develop and demonstrate the usefulness of a new power toothbrush, one that incorporated both sonic processes (in the form of rapid bristle motion that directly removes plaque as well as generates bubbles) and ultrasound (sourced from the brush head, that activated the bubbles). We adapted a set of existing power toothbrushes to incorporate an ultrasound source with sufficient controls to explore the effects of different ultrasound parameters on plaque removal in vitro and in vivo. The combination of sonic and ultrasound physics removed plaque in vitro in a synergistic manner, likely through the action of cavitation. In vivo, the Ultreo toothbrush - a commercial version of this brush-removed comparable amounts of plaque to another commercial brush in half the time, and removed more plaque than manual toothbrushes. A combination of sonic bristle motion and ultrasound-facilitated cavitation appears to produce superior plaque removal compared to existing, sonic only, or manual tooth brushes.

\section{9:45}

2aPAa8. Motion of dissolved gas in a cavitating fluid. I. V. Mastikhin, B. Newling, and S. Kristoffersen (Dept. of Phys., Univ. of New Brunswick, 8 Bailey Dr., Fredericton, New Brunswick, E3B 5A3, Canada)

A strong acoustic field in a liquid separates the liquid and dissolved gases by the formation of bubbles (cavitation). Bubble growth and collapse is the result of active exchange of gas and vapor through the bubble walls with the surrounding liquid. This work investigates a new approach to the study of cavitation, not as an evolution of discrete bubbles, but as the dynamics of molecules constituting both the bubbles and the fluid. Direct Magnetic Resonance Imaging measurements of dynamics of the liquid and the dissolved gas show that the motions of dissolved gas (freon-22, CHClF2) and liquid (water) can be very different during acoustic cavitation and are strongly affected by the ultrasonic power, filtration, or previous cavitation of the solvent. The observations suggest that bubbles can completely refresh their content within two acoustic cycles and that long-lived $(\sim$ minutes $)$ mi- 
crobubbles act as nucleation sites for cavitation. The technique is complementary to the traditional optical and acoustical techniques. [V. Mastikhin and B. Newling, Physical Review E $(2005,2008)$.] [Authors thank the Natural Science and Engineering Research Council of Canada for funding.]

\section{0:00}

2aPAa9. Simultaneous, noninvasive determination of fluid viscosity and density from acoustical resonances. Curtis F. Osterhoudt and Dipen N. Sinha (Los Alamos Natl. Lab., MPA-11 MS D429, Los Alamos, NM 87544, sinha@lanl.gov)

U-tube densitometers make fluid density measurements based on eigenfrequency shifts, which depend on the fluids contained within the densitometer. This measurement method is the current standard for density measurements in industrialized settings. However, ruggedized densitometers have several drawbacks. They are expensive, and require diverting fluids into the apparatus to make their measurements. Viscometers, too, are considered invasive, or require diverting fluid into a measurement chamber (the one exception is the "OSCAR" viscometer, which cannot make measurements on flowing systems). In contrast, a simultaneous density/viscosity measurement can be made without diverting fluid from the container or pipe carrying it. The measurement, in which the container (e.g., pipe section) itself becomes the sensor, is based on acoustical frequency resonance shifts and resonance width changes. Such measurements can in principle be made at a standoff from the container, reducing price and requiring no further contact with the fluids. This presentation will address the technique, give the theoretical background for it, and present experimental validation.

TUESDAY MORNING, 19 MAY 2009

BROADWAY III/IV, 10:30 A.M. TO 12:00 NOON

\title{
Session 2aPAb
}

\section{Physical Acoustics: Thermoacoustics}

\author{
Martin D. Verweij, Chair \\ Lab. for Electromagnetics Research, Delft Univ. of Technology, 2628 CD Delft, The Netherlands
}

\section{Contributed Papers}

10:30

2aPAb1. Thermoacoustics in irregular media. Carl Jensen and Richard Raspet (Natl. Ctr. for Physical Acoust., Univ. of Mississippi, University, MS 38677)

One approach to acoustics in random porous media is to use fitting parameters to match theoretical models derived from exact parallel pore theory to measured values of the media's acoustic properties such as the complex compressibility and complex density. [Roh et al. J. Acoust. Soc. Am., 121, 1413-1422 (2007)] extended these ideas to the more complicated thermoacoustic case of an applied temperature gradient by using empirical factors to reshape the known solution for thermoacoustics in a regular framework of rigid tubes to RVC and aluminum foam samples. The validity of this model has been investigated by directly simulating the sound propagation through several irregular, 3-D geometries using a thermal fluid solver. The acoustic and thermoacoustic simulation results for several geometries will be presented and compared to the theoretical results.

\section{0:45}

2aPAb2. Helmholtz-like resonators for thermoacoustic prime movers. Bonnie J. Andersen (Dept. of Phys., Utah Valley Univ., 500 W. University Pkwy., Orem, UT 84058) and Orest G. Symko (Univ. of Utah, Salt Lake City, UT 84112)

In a thermoacoustic prime mover, high acoustic output power can be achieved with a large diameter stack and with a cavity with a large volume attached at the open end of the resonator containing the stack. The combination of resonator and cavity makes the device Helmholtz-like, with special characteristics of the resonant frequencies and quality factor $Q$. Analysis of its acoustic behavior based on a model of a closed bottle presents features that are useful for the development of such prime movers for energy conversion from heat to sound. In particular, the arrangement produces in the cavity a high sound level, which is determined by the $Q$ of the system. Comparison with a half-wave resonator type of prime mover, closed at both ends, shows the advantages of the Helmholtz-like device.

\section{1:00}

2aPAb3. Ultrasonic thermoacoustic prime movers. Myra Flitcroft and Orest G. Symko (Dept. of Phys., Univ. of Utah, 115 S. 1400 E., Salt Lake City, UT 84112)
Thermoacoustic devices that generate sound with frequencies in the near ultrasonic range $(\sim 21 \mathrm{kHz})$ have been developed. These devices consist of a wave resonator that is $4.1 \mathrm{~mm}$ in length, with hot and cold heat exchangers made of copper mesh placed at the resonator's midpoint. Between these is sandwiched a stainless steel stack; the working fluid is air at 1 atmosphere. Heat is injected to the hot heat exchanger, creating a temperature gradient across the stack. When this exceeds a critical temperature gradient, acoustic oscillations ensue with intensities over $140 \mathrm{~dB}$. The performance of a given engine depends drastically on the alignment achieved in the assembly process. Due to this variability, temperature differences at onset ranged from $85^{\circ} \mathrm{C}$ to $180^{\circ} \mathrm{C}$. In order to increase positive feedback in the systems, single engines were coupled to a resonant cavity, the end of which was fitted with a pressure transducer. The use of a cavity also provides an acoustic coupling mechanism in which two ultrasonic engines can be attached to the same cavity, allowing for the investigation of synchronization of these devices in this regime. [Research supported by the U.S. Army Space and Missile Defense Command/U.S. Army Forces Strategic Command.]

\section{1:15}

2aPAb4. Miniature traveling wave thermoacoustic engine. Ivan A. Rodriguez and Orest G. Symko (Dept. of Phys., Univ. of Utah, 115 S. 1400 E., Salt Lake City, UT 84112)

Five high frequency annular thermoacoustic traveling wave devices have been developed and characterized. A $1.27 \mathrm{~cm}$ bore diam $2 \mathrm{kHz}$ engine was optimized, increasing its maximum acoustic output from 140 to $167 \mathrm{~dB}$. A new $1.60 \mathrm{~cm}$ bore diam $2 \mathrm{kHz}$ engine has been assembled with the expectation of even higher acoustical power output because power is proportional to cross-sectional area. In the $3 \mathrm{kHz}$ regime, two geometries were investigated, an oval shape and more square version, which is easier to assemble. These devices were found to have comparable outputs suggesting that the square geometry is a viable alternative, and, thus, all additional devices were constructed this way. A $4 \mathrm{kHz}$ engine was also studied. In order to measure the effects of the heat exchangers and regenerator assembly on the acoustic flow, a resonator tube was constructed. This tube was fitted with the device innards and driven by a speaker. An absorption coefficient of 0.4 was measured. This information along with the $916 \mathrm{~T}$ across the regenerator allows for an estimate of the limit on the acoustic gain of the system to be 
calculated. [Research supported by the U.S. Army Space and Missile Defense Command/U.S. Army Forces Strategic Command.]

\section{1:30}

2aPAb5. Particle image velocimetry study of acoustic field in miniature traveling wave device. Ivan A. Rodriguez and Orest G. Symko (Dept. of Phys., Univ. of Utah, 115 S. 1400 E, Salt Lake City, UT 84112)

Particle imaging velocimetry (PIV) techniques were employed to investigate the flow patterns inside a $2 \mathrm{kHz}$ annular thermoacoustic device. This thermoacoustic device uses air at one atmosphere as its working fluid and smoke for seeding particles. For these measurements, the sound level output of the device was kept to $140 \mathrm{~dB}$ to help maintain the suspension of seeding particles. Several types of flow patterns were observed, including Gedeon streaming, start-up noise characterized by instabilities such as vortices, and a traveling wave throughout accompanied by radial standing waves localized near corners. Such measurements are challenging in small high frequency devices. In order to satisfy the Shannon-Nyquist theorem, a $2 \mathrm{kHz}$ engine would need to be sampled at a minimum of $4 \mathrm{kHz}$; however, a new technique was developed that allows for the measurements to be made using a camera with a maximum frame rate per sec of 300. This new method makes it possible to perform measurements on a flow without the need for more expensive and delicate high speed equipment, thereby, making observation of high speed flows more readily available. [Research supported by the U.S. Army Space and Missile Defense Command/U.S. Army Forces Strategic Command.]
11:45

2aPAb6. Coupling of midaudio frequency thermoacoustic prime movers. Brenna G. McDonald and Orest G. Symko (Dept. of Phys., Univ. of Utah, 115 S. 1400 E., Salt Lake City, UT 84112)

Multiple unit midaudio frequency thermoacoustic engine arrays have been studied in terms of synchronized self-sustaining oscillators. Thermoacoustic engines can be acoustically coupled through shared gas when attached to a common cavity. The coupling between devices was studied by varying the cavity volume, and by varying the separation distance between devices in an array. Frequency and phase measurements for two-engine arrays have suggested that the engines entrain at a common frequency with approximately zero phase difference implying the onset of synchronization. Amplitude measurements of synchronized arrays suggest that the acoustic amplitude is equal to the sum of the amplitudes of each uncoupled engine, and agree with predictions. Synchronization of four or more units in an array implies that the coupling between engines is global and depends on the volume of the cavity and the density of the gas within the cavity. The detuning between two engines was increased until synchronization no longer occurred. The threshold for this was found to be for a frequency difference of between 80 and $98 \mathrm{~Hz}$ for the given coupling strength when operating near $2.5 \mathrm{kHz}$. [Research supported by the U.S. Army Space and Missile Defense Command/U.S. Army Forces Strategic Command.]

\title{
Session 2aPP
}

\section{Psychological and Physiological Acoustics: Theory Construction in the Domain of Auditory Perception}

\author{
Frederick J. Gallun, Cochair \\ National Ctr. for Rehabilitative Audiology Research, Portland VA Medical Center, Portland, OR 97239-2964 \\ Nathaniel I. Durlach, Cochair \\ Research Laboratory of Electronics, Massachusetts Inst. of Technology, Cambridge, MA 02139
}

Chair's Introduction-8:30

\section{Invited Papers}

$8: 35$

2aPP1. Shampooing, skydiving, and the cochlear amplifier. Christopher A. Shera (Eaton-Peabody Lab., 243 Charles St., Boston, MA 02114, shera@epl.meei.harvard.edu)

What is the evidence that the mammalian cochlea amplifies traveling waves? Although mechanical nonlinearity and physiological lability are both widely cited as such, neither constitutes evidence for actual power amplification. In fact, the only real evidence comes from cochlear modeling. In this presentation I describe two modeling strategies, "shampooing" and "skydiving;" discuss the relative strengths and weaknesses of the two approaches (more conventionally dubbed the forward and inverse method, respectively); and illustrate their application to cochlear mechanics. Skydiving with a parachute provides compelling evidence that the mammalian cochlea operates as a biological, hydromechanical analog of a laser amplifier. [Work supported by the NIH/NIDCD.]

\section{8:55}

2aPP2. Development of a revised loudness model. Brian C. J. Moore (Dept. of Experimental Psychol., Univ. of Cambridge, Downing St., Cambridge CB3 9LG, UK, bcjm@cam.ac.uk)

The loudness model developed in my laboratory was based on models developed by pioneers such as Fletcher and Zwicker. Their models involved calculation of an excitation pattern for the sound of interest, from the long-term average spectrum of the sound, transformation of the excitation pattern to a loudness pattern (specific loudness plotted as a function of frequency on a perceptually based 
frequency scale), and calculation of loudness from the area under the loudness pattern. The innovations used in the Cambridge model were: (1) Incorporation of a linear filter as a first stage, to account for the transmission of sound through the outer and middle ear; (2) Calculation of excitation patterns from extensive measurements of auditory filter shapes, using the notched-noise method; (3) Transformation of the frequency scale to the $E R B_{N}$-number scale, which is similar conceptually to the Bark scale, but is different numerically, especially at low frequencies; (4) Transformation of excitation to specific loudness using a function such that loudness has a finite value at the absolute threshold, as opposed to zero loudness. More recently, the model has been modified to include the concept of binaural inhibition, to accommodate experimental evidence indicating that loudness does not simply sum across ears.

\section{9:15}

2aPP3. Auditory perception through the cortical analysis of spectrotemporal modulations. Shihab Shamma (Dept. of Elect. and Comput. Eng., Inst. for Syst. Res., Univ. of Maryland, College Park, MD 20742)

To understand the functional organization of the primary auditory cortex (A1), a broad array of stimuli have been used over the years ranging from simple tones to species-specific vocalizations. In our experience, broadband stimuli with spectrotemporally modulated spectra have been most effective in evoking vigorous cortical responses and facilitating measurements of spectrotemporal receptive fields (STRFs). Extensive measurements with such stimuli led us to conclude that A1 cells analyze the spectrotemporal modulation content of their input stimulus through a rich assortment of STRFs spanning the dynamically slow to fast, the spectrally narrow to broad, and tuned at all best frequencies. These experimental findings have subsequently been formulated as a "multiresolution cortical model" whose output is postulated to represent the perceptually significant elements of sound. I shall briefly describe how complex cortical responses have been abstracted and interpreted in the context of this model, and how the model has been effective in a wide variety of applications including the assessment of speech intelligibility, representation of phonemes, explaining the perception of monaural phase sensitivity, detecting speech versus nonspeech sounds, quantifying musical timbre, enhancement of noisy speech, and in the analysis and organization of complex auditory scenes.

\section{9:35}

2aPP4. Binaural interaction modeling based on physiology. H. Steven Colburn (Dept. of Biomed. Eng., Hearing Res. Ctr., Boston Univ., 44 Cummington St., Boston, MA 02215, colburn@bu.edu)

Since psychophysical performance is based on information encoded by peripheral sensory receptors, this encoding and the resulting internal representation of sensory information impose limitations on psychophysical abilities. When enough knowledge and data are available to characterize the activity of the neurons carrying the relevant information, it is possible to derive implications for performance and constraints on mechanisms of performance. This approach has been applied for many years to multiple sensory modalities including vision and audition [e.g., Siebert, Kybernetik (1965)]. In this presentation, the impact of these ideas on understanding binaural processing will be discussed. As argued in many talks in this session, explicit models with the potential for quantitative predictions are an effective means to represent current understanding to explore hypotheses. [Work supported by NIDCD, grant No. DC-00100.]

\section{9:55-10:10 Break}

\section{0:10}

2aPP5. Autocorrelation models of pitch perception. William A. Yost (Speech and Hearing Sci. , ASU, P.O. Box 870102, Tempe, AZ 85287-0102, william.yost@asu.edu)

In 1951, Licklider first suggested autocorrelation as a method to model pitch perception in his Duplex and Triplex Theories. It was not until 1991 that a full-fledged autocorrelation model was developed by Meddis and Hewitt. Over the past decades several investigators have refined autocorrelation or autocorrelation-like models to account for pitch perception and processing. While there have been a few arguments against autocorrelation for explaining pitch phenomena, autocorrelationlike models have been very successful in accounting for a wide range of data including those associated with the pitch of iterated ripple noise. This presentation will review the development of autocorrelation models of pitch perception, especially in accounting for the pitch and pitch strength of iterated ripple noise. The presentation will also cover how refining well-established models or theories may offer a good opportunity for developing skills to build additional models and theories. [Work supported by a grant from NIDCD.]

\section{0:30}

2aPP6. Theories of hearing. Neal F. Viemeister (Dept. of Psych., Univ. of Minnesota, Minneapolis, MN 55455, nfv@umn.edu)

One of the more compelling and important aspects of auditory science has been the ability to relate perception to physiology and physics. This challenging endeavor has attracted fine minds from various disciplines and has provided theoretical frameworks for rigorously studying and describing at various levels many basic aspects of hearing. An example is the application of "signal detection theory" and its collection of tools for empirical and theoretical investigation of certain aspects of audition. But what of the future? Do we have comparable tools to construct quantitative theories that enable prediction of complex aspects of audition? Do we need new tools? Definitive answers may or may not be provided during this session.

\section{0:50}

2aPP7. Relevance of psychoacoustical theory. William Hartmann (Dept. of Phys. and Astron., Michigan State Univ., 1226 BPS Bldg., East Lansing, MI 48824)

Historically, the principles of psychoacoustics have been described in terms of mathematical theories. Are such mathematical theories now obsolete? Have they been replaced by computer algorithms? Do contemporary answers now come from simulations in computational toolboxes? The case for psychoacoustical theory can be made anew: Mathematical psychoacoustical theory has value because 
of the transparency of its assumptions and the universality of its language. It has an unparalleled ability to inspire extensions and revisions in order to evolve. It illuminates new conceptual directions through analogies with other sciences subserved by similar mathematical structures. Theory consolidates large amounts of otherwise unrelated data and serves as a guide to future experiments. Mathematical theory is the practical realization of images about the way the auditory world works, images that promote understanding because of their intuitive appeal. Theory is at its best when the ordinary operations of mathematics transform an initial model into logically inescapable new insights unlikely to have arisen by other avenues. [Work supported by the NIDCD, Grant DC-00181.]

11:10-11:45 Panel Discussion

TUESDAY MORNING, 19 MAY 2009

FORUM SUITE, 8:40 TO 11:55 A.M.

\title{
Session 2aSA
}

\section{Structural Acoustics and Vibration: Computational Structural Acoustics}

\author{
Kuangcheng $\mathrm{Wu}$, Chair \\ Signatures \& Hydrodynamics, Northrop Grumman Newport News, Newport News, VA 23607-2770
}

\section{Invited Papers}

\author{
$8: 40$
}

2aSA1. A three-dimensional Wiener-Hopf technique for general bodies of revolution, Part I: Symmetrization and factorization of the integral equation's kernel. Rudolph Martinez and Carina Ting (CAA-Alion Corp., 84 Sherman St., Cambridge, MA 02140, rmartinez@alionscience.com)

This is the first of a two-part paper on the development of analytic solutions of acoustic scattering and/or radiation from arbitrary bodies of revolution under heavy fluid loading. The approach followed is the construction of a 3-D Wiener-Hopf technique with Fourier transforms that operate on the finite object's arclength variable (the object's practical finiteness comes about, in a Wiener-Hopf sense, by formally bringing to zero the radius of its semi-infinite generator curve for points beyond a prescribed station). Unlike in the classical case of a planar semi-infinite geometry, the kernel of the integral equation is nontranslational and therefore with independent wavenumber spectra for its receiver and source arclengths. The solution procedure applies a symmetrizing spatial operator that reconciles the regions of $(+)$ and $(-)$ analyticity of the kernel's two-wavenumber transform with those of the virtual sources. The spatially symmetrized integral equation is of the Fredholm second kind and thus with a strong unit "diagonal"-a feature that makes possible the Wiener-Hopf factorization of its transcendental doubly transformed kernel via secondary spectral manipulations. The second of this two-part paper describes how the theory addresses departures from axisymmetry through substructuring techniques and demonstrates the analysis for canonical problems of fluid-structure interaction.

\section{9:00}

2aSA2. Midfrequency acoustic radiation analysis. Stephen D. O'Regan (Naval Surface Warfare Ctr., Carderock Div. 9500 MacArthur Blvd., West Bethesda, MD 20816, Stephen.ORegan@navy.mil)

Boundary, finite, and infinite element methods have proven useful for solving the 3-D acoustic wave equation at low frequencies. For high frequency, localized formulations such as plane-wave and ray-tracing methods have been applied successfully. These methods are impractical, inaccurate, or both for treating midfrequency problems. Low-frequency methods make very large demands on computer processing while high-frequency methods account only for local effects. Full-spectrum methods have been developed which apply interpolation between the low and high ends of the frequency range to estimate the response in the midfrequency. While these methods have provided some insight to trends as one moves toward the midfrequency range, it is not well established that they can reliably solve problems well away from their established limits. For surfaces with features having dimensions close to midfrequency wavelengths, the physics is distinct from those that govern low and high frequencies, and is not merely a linear combination of the two. This presentation reports on research to develop a midfrequency analysis tool that directly addresses the midfrequency physics. The author will identify the challenges in a physics-based method, discuss an approach to address them, and report on simulations exercising the approach.

$$
\text { 9:20 }
$$

2aSA3. The discontinuous enrichment method for multiscale and higher-frequency wave propagation problems. Charbel Farhat and Radek Tezaur (Dept. of Aeronaut. and Astronaut., 496 Lomita Mall, Stanford Univ., Stanford, CA 94305-4035)

Wave propagation problems pertain to many technologies including sonar, radar, geophysical exploration, medical imaging, nondestructive testing, and structural design. In the medium frequency regime, the analysis of these problems by the standard finite element method is either computationally unfeasible or simply unreliable, because of the well-known pollution effect. In practice, the higherorder (or $p$-type) finite element method alleviates this effect, but only to some extent. Alternative approximation methods based on plane waves have recently emerged to address this issue. The discontinuous enrichment method (DEM) is such an alternative. It distinguishes 
itself from similar approaches by its ability to evaluate the important system matrices analytically, thereby bypassing the typical accuracy and cost issues associated with high-order quadrature rules. DEM also provides a unique multiscale approach to computation by employing fine scales that contain solutions of the underlying homogeneous partial differential equation in a discontinuous framework. The theoretical and computational underpinnings of this method will be overviewed. Then, recent applications to underwater acoustic and elastoacoustic scattering as well as automotive structural vibrations in the midfrequency regime will be discussed. Accuracy of 1 to 2 orders of magnitude and/or solution time improvement over the higher-order Galerkin method will be demonstrated in three dimensions.

9:40

2aSA4. Eigenanalysis in structural acoustics. Jeffrey L. Cipolla (Weidlinger Assoc. Inc., 375 Hudson St., New York, NY 10014)

Determination of characteristic frequencies and solutions (mode shapes) for vibrating systems is a staple of linear dynamics in engineering, so it should be no surprise that analysts strive for the same in coupled structural-acoustic systems. Computation of these eigensolutions confers not only physical insight into the behavior of the system, but also a means to reduce the mathematical dimension of frequency-response and other engineering computations. As in other mechanical vibration problems, the eigensolutions of coupled systems form a nearly optimal space for the approximation of the forced-response solutions. However, the coupled structural-acoustic eigenproblem is not trivial, and can be prohibitively expensive if not handled properly; this is particularly true for exterior problems. Notably, identification of a physically pertinent self-adjoint operator for the system of interest is a critical challenge. Here we will discuss the coupled structural-acoustic eigenproblem, various approximations common in practice, as well as alternative approaches to model reduction.

\section{0:00}

2aSA5. Recent innovations and current performance of conventional boundary element computations. John B. Fahnline (Water Tunnel Bldg., The Pennsylvania State Univ., University Park, PA 16802)

As long as boundary element methods have existed, research has been ongoing to make the calculations faster and applicable for larger problems. The rapid rise in computing power, both in terms of processor speed and storage capacity, has increased the problem size that can be addressed and has led to many novel strategies for reducing the computational burden. For example, many of the matrices can be stored at coarse frequency spacing over the range of interest and interpolated at much finer resolution, reducing the computation times enormously. Other strategies for speeding the computations that will be discussed include condensed acoustic meshes, optimized math kernel libraries, and multipole expansions. Results will be presented to indicate typical computation times and problem size limitations.

\section{0:20—10:40 Break}

\section{Contributed Papers}

\section{0:40}

2aSA6. Sonic boom propagation into urban landscapes: Preliminary study. Kimberly Lefkowitz (CSTI Acoust., 15835 Park Ten Pl. Ste. 105, Houston, TX 77084, kim@cstiacoustics.com) and Victor W. Sparrow (Penn State Univ., University Park, PA 16802)

In order to characterize the sonic boom response in the interiors of buildings in urban settings, first the loading forces impinging on such buildings must be understood. To do this in an urban setting, many factors must be taken into account including the shape and surface structure of the building of interest, the kind of boom being propagated, and the scale of the propagation space. Nearby buildings and their features also require consideration. In addition, the importance of specular reflection versus diffuse reflections should be addressed. In this study, these factors are discussed and evaluated relative to the impact on the overall sound field. Preliminary results on a simple case are presented. [Work supported by NASA.]

\section{0:55}

2aSA7. Wideband fast multipole accelerated boundary element methods for the three-dimensional Helmholtz equation. Nail A. Gumerov and Ramani Duraiswami (Perceptual Interfaces and Reality Lab., UMIACS \& Comput. Sci. Univ. of Maryland, College Park, MD 20742)

The development of a fast multipole method (FMM) accelerated iterative solution of the boundary element method (BEM) for the Helmholtz equations in three dimensions is described. The FMM for the Helmholtz equation is significantly different for problems with low and high $k D$, where $k$ is the wave number and $D$ the domain size, and for large problems, the method must be switched between levels of the hierarchy. The BEM requires several approximate computations: numerical quadrature and approximations of the boundary shapes using elements. These errors must be balanced against approximations introduced by the FMM and the conver- gence criterion for an iterative solution. These different errors must all be chosen in a way that, on the one hand, excess work is not done and, on the other, that the error achieved by the overall computation is acceptable. Details of translation operators for low and high $k D$ choice of representations and BEM quadrature schemes, all consistent with these approximations, are described. A novel preconditioner using a low accuracy FMM accelerated solver as a right preconditioner is also described. Results of the developed solvers for large boundary value problems with $0.0001 \leqslant k D \leqslant 500$ are presented and shown to perform close to theoretical expectations.

\section{1:10}

2aSA8. Energy flux streamlines vs the alternatives for the visualization of energy coupling at and inside the surface of an ensonified fluid loaded elastic cylindrical shell with vacuum interior: Preliminary results. Cleon E. Dean (Phys. Dept., P.O.B. 8031, Georgia Southern Univ., Statesboro, GA 30460-8031, cdean @GeorgiaSouthern.edu) and James P. Braselton (Georgia Southern Univ., Statesboro, GA 30460-8093)

Energy flux streamlines are compared with alternative presentations of the energy flux vector field to visualize energy coupling at the surface and inside an ensonified fluid loaded elastic cylindrical shell with vacuum interior for both forward and retrograde guided propagating waves. The present work uses a method adapted from a simpler technique due to Kaduchak and Marston [Gregory Kaduchak and Philip L. Marston, "Traveling-wave decomposition of surface displacements associated with scattering by a cylindrical shell: Numerical evaluation displaying guided forward and backward wave properties," J. Acoust. Soc. Am. 98, 3501-3507 (1995)] to isolate unidirectional energy flows. 


\section{$11: 25$}

2aSA9. Locating arbitrary noise sources in three-dimensional space in real time. Na Zhu (Dept. of Mech. Eng., Wayne State Univ., 5050 Anthony Wayne Dr., Detroit, MI 48202 dv6930@wayne.edu) and Sean Wu (Wayne State Univ., Detroit, MI 48202)

A portable, easy to use and effective device using four microphones only is developed for locating arbitrary noise sources in 3-D space in real time. The microphones can be placed anywhere in space so long as they are not on the same plane. This provides application flexibilities. Using crosscorrelations, the differences in arrival times of these four microphone channels can be specified. This information together with a spherical spreading assumption of acoustic wave traveling in a free field enables one to determine the $(x, y, z)$ coordinates of target sources. Error analyses are conducted and the impacts of signal noise ratios, microphone spacing, source distances, and frequencies on the accuracy of localization are examined. In particular, an empirical formulation for estimating the errors in locating a target source is developed. Using this empirical formulation, one can locate a target source for any given signal noise ratio, microphone spacing, source distance, and frequency range. Numerical simulations and experimental validations are conducted to check the effectiveness of this device to locate a variety of real world sounds, including truck pass-by noise, thundering, human voices, etc. Satisfactory results are obtained in most cases, even when the sources are behind the measurement microphones.

\section{1:40}

2aSA10. Low frequency radiation from a (compact) structure with and without sound holes. Kyle Martini (84 Sherman St., Cambridge, MA 02140,kmartini@alionscience.com)

At long wavelengths, a force driven, closed elastic structure radiates as a dipole. However, this may or may not be the case in the presence of a sound hole. Radiation remains dipole-like when the interior volume behaves as an acoustic fluid coupled to the structure. This is commonly referred to as the sound hole sum rule in musical acoustics [Weinreich (1985)]. However, should the interior acoustic volume be uncoupled, or simply ignored, the radiated field is dominated by a monopole contribution. For example, this may occur with mechanically isolated, piping systems exposed to the exterior medium. In this situation, the monopole or dipole nature of the radiation depends on the effective number of sound holes, for example, whether the driven system is well connected to the exterior along both the inlet and outlet. Finite element structural-acoustic models are developed and exercised to investigate these systems and issues. In addition, a substructuring technique is utilized to account for the potential influences of interior structural complexity.

TUESDAY MORNING, 19 MAY 2009

GRAND BALLROOM I, 8:00 A.M. TO 12:00 NOON

\title{
Session 2aSC
}

\section{Speech Communication: Phonetics and Phonology (Poster Session)}

\author{
Ratree Wayland, Chair \\ Program in Linguistics, Univ. of Florida, Gainesville, FL 32611-5454
}

\section{Contributed Papers}

\begin{abstract}
All posters will be on display from 8:00 a.m. to 12:00 noon. To allow contributors an opportunity to see other posters, contributors of odd-numbered papers will be at their posters from 8:00 a.m. to 10:00 a.m. and contributors of even-numbered papers will be at their posters from 10:00 a.m. to 12:00 noon
\end{abstract}

2aSC1. The perception of word-final nasals in Southern Min and Mandarin Chinese. Ying Chen and Susan G. Guion (Dept. of Linguist., 1290 Univ. of Oregon, Eugene, OR 97403, ychen12@uoregon.edu)

There is a historical pattern of word-final nasal merger in Chinese languages. Some languages preserve the old Chinese final nasals /m, n, y/, others preserve only $/ \mathrm{n}, \mathrm{y} /$, while others preserve only $/ \mathrm{y} /$. The pattern of merger and loss, $/ \mathrm{m} />/ \mathrm{n} />/ \mathrm{n} /$, may be due to a hierarchy of confusability among the nasals. Here, relative confusability of nasals was examined in identification tasks. Effects of vowel quality and lexical tone were also considered. First, perception of final nasals in the Quanzhou dialect of Southern Min, which preserves $/ \mathrm{m}, \mathrm{n}, \mathrm{y} /$, found that $/ \mathrm{m} /$ was most often confused, while /n/ was least often confused. High vowel /i/ conditioned more mishearing than mid vowel /a/ and low vowel /a/; low-level tone conditioned more mishearing than high-level, midlevel, midlow-rising and midhigh-falling tones. Second, perception of final nasals in Mandarin, which preserves $/ \mathrm{n}, \mathrm{y} /$, found that $/ \mathrm{n} /$ was more often confused than $/ \mathrm{y} /$. High vowel /i/ again conditioned more mishearing than / $/ /$ and /a/; low-dipping tone conditioned more mishearing than high-level, midrising, and highfalling tones. The results do not strongly support the hierarchy of confusability among the nasals but suggest that high vowels and low tones condition more misidentifications.

2aSC2. Velarization of word-initial /// in African American English. Janneke Van Hofwegen and Erik R. Thomas (Dept. of English, North Carolina State Univ., Box 8105, Raleigh, NC 27695-8105, ethomas@ social.chass .ncsu.edu)
Word-initial /1/ was examined in African American English in North Carolina, with comparisons with European American English, archival interviews with ex-slave African Americans and Spanish. The degree of velarization was measured using the F2-F1 difference in Bark. Interactions with formant values of the following vowel and the duration of $/ 1 /$ were examined. Past research has produced conflicting results. First, initial /l/ in English is regarded as relatively unvelarized, but some research has found it to be velar. Second, there is disagreement about whether velar or nonvelar /1/ shows greater duration. Third, there has been controversy over whether velarization of /l/ is categorical or scalar. Results showed that English speakers exhibited significantly more velarization than Spanish speakers. Moreover, the degree of velarization increased across generations of African Americans. Less velarized /// proved to show greater duration than more velarized /1/. Velarization was quite scalar: there was no discernable division between more and less velarized tokens. $/ 1 /$ tended to be more velar before a back vowel than before a front vowel, but this correlation showed no interaction with the observed generational and individual differences in velarization. [Work supported by NSF.]

2aSC3. An acoustic study of $/ \mathbf{w} /$ in Korean. Sun-Ah Jun and Jason B. Bishop (Dept. of Linguist., UCLA, Los Angeles, CA 90095-1543)

This study examines acoustic characteristics of Korean /w/. Modern Korean is claimed to have four /w/ diphthongs: /w/ before two front vowels (/wi, we/) and two back vowels (/wa, ws/). The two front diphthongs are a product of a more recent sound change from front rounded monophthongs, high $/ y /$ and mid $/ \varnothing /$. However, a pilot study shows that the phonetic real- 
izations of $/ \mathrm{w} /$ before front vowels differ from those before back vowels, and also differ whether $/ \mathrm{w} /$ comes after a consonant $(\mathrm{CwV})$ or not $(\mathrm{wV})$. It was found that $/ \mathrm{w} /$ before front vowels is similar to a labial-palatal approximant, especially after a consonant, showing a trace of front rounded monophthongs. To confirm this finding, a large set of data was collected from six Korean speakers and the acoustic realizations of Korean /w/ were compared with those of French labial-palatal and labial-velar approximants produced by six French speakers. The implication of the results in the phonological system will be discussed.

2aSC4. Sound change and perceptual weight of acoustic correlates to Korean stops. Kyoung-Ho Kang and Susan Guion (Dept. of Linguist., Univ. of Oregon, 1290 Univ. of Oregon, Eugene, OR 97403)

The Korean stop system is experiencing a sound change. Younger Korean speakers born in the 1970s or after produce aspirated and lenis stops differently from older speakers. Results from clear speech production experiments revealed differences in clear speech enhancement patterns. Older speakers enhanced voice onset time (VOT) difference, whereas younger speakers primarily enhanced F0 difference [K.-H. Kang and S. G. Guion, J. Acoust. Soc. Am. 124, 3909-3917 (2008)]. These results indicate that older and younger speakers have different phonetic targets for the production of stops. The primary goal of the current study was to examine any perceptual differences related to the production differences. In order to assess the extent to which acoustic correlates to the stops contribute to the identification of the Korean stops, a perception experiment using resynthesized stimuli was conducted. Younger $(n=10$, mean of 20.2 years old) and older listeners $(n=$ 10 , mean of 48.5 years old) participated in a stop identification task. The results suggested that F0 is a more weighted correlate than VOT for younger listeners for the distinction of the aspirated and lenis stops.

2aSC5. Phonation categories in Cajonos Zapotec. Laura Tejada (Dept. of Linguist., Univ. of So. California, GFS 301, Los Angeles, CA 90089, 1tejada @usc.edu)

Phonation categories such as breathy, modal, and creaky have been described using the acoustic measurements $(\mathrm{H} 1-\mathrm{H} 2)$ and $(\mathrm{H} 1-\mathrm{A} 1)$. The measure $(\mathrm{H} 1-\mathrm{H} 2)$ is the difference in $\mathrm{dB}$ between the first and second harmonics and is used to approximate the proportion of a glottal cycle in which the glottis is open (the open quotient). (H1-A1) is the difference in $\mathrm{dB}$ between the first harmonic and the most prominent harmonic in the F1 region. (H1-A1) is used as a measure of F1 bandwidth that is related to degree of glottal opening. These measures were applied to an undocumented variety of Villa Alta Zapotec spoken in San Miguel Cajonos. Acoustic data from four speakers were analyzed. Measures were taken at four evenly spaced points in time for /i/, /e/, /a/, and /o/. Results show that (H1-H2) and (H1-A1) discriminated correctly between the categories breathy, modal, and creaky with varying percentages of accuracy at different time points. (H1-H2) had a $(58 \%)$ accuracy rate at time point three, compared with $(63 \%)$ for (H1-A1). Combining the two measures showed that the accuracy rates rose from point one $(50 \%)$ through points two $(61 \%)$ and three $(65 \%)$, and fell slightly at point four $(58 \%)$.

2aSC6. The phonetics of contrastive phonation in Gujarati. Sameer ud Dowla Khan (Pitzer College, 1050 N. Mills Ave., Claremont, CA 91711, and Dept. of Linguist., 3125 Campbell Hall, UCLA, Los Angeles, CA 90095) and Victoria Thatte (UCLA, Los Angeles, CA 90095)

Gujarati is known for distinguishing breathy and modal phonation in both consonants and vowels [(Cardona and Suthar, 2003)], e.g., /ba:r/ "twelve" versus /Ba:r/ "burden" versus /bA:r/ "outside," where uppercase represents breathiness. The current study investigates acoustic and glottographic properties of this three-way contrast. [Fischer-Jørgensen (1967)] and [Bickley (1982)] showed that the H1-H2 measure can reliably distinguish breathy and modal vowels, and [Esposito (2006)] further found that Gujarati listeners exclusively attend to $\mathrm{H} 1-\mathrm{H} 2$ to categorize audio samples from other languages, even when H1-H2 did not reliably distinguish contrastive voice quality in those languages. However, do other acoustic properties also distinguish breathiness in Gujarati? In addition what physiological differences underlie the acoustic differences? The current study tests the reliability of several phonetic measures (e.g., H1-H2, H1-A1, CPP, CQ, etc.) to distinguish 33 (near-) minimal sets contrasting breathy and modal segments in audio and electroglottographic data produced by both male and female Gujarati speakers. [Work supported by NSF].

2aSC7. The effect of boundary recoverability on geminate length in English. Grace E. Oh and Melissa A. Redford (Dept. of Linguist., 1290 Univ. of Oregon, 1451 Onyx St., Eugene, OR 97403)

[Payne (2005)] argues that fake geminates are longer than true geminates, and [Ridouane (2007)] shows that their preceding vowel duration is also significantly longer. These effects are likely due to the boundary that distinguishes fake from true geminates. If this is correct, then we might expect that boundary strength will also affect geminate realization. We investigated this possibility by comparing the absolute and relative durations of assimilated and concatenated word-internal and cross-word boundary fake geminates in English (e.g., immoral versus unnamed versus fun name). Eight speakers produced five repetitions of 32 stimuli (four types: three geminate, one singleton) in two speech styles. The results showed that although cross-boundary geminates were longer than word-internal concatenated and assimilated geminates in absolute terms, both types of concatenated geminates were shorter than assimilated geminates in relative terms. A follow up experiment, comparing geminates in compound words to those emerging across words showed no differences in relative duration between the two. These results suggest that boundary strength may be less important than boundary recoverability in production. Overall, we argue that boundary recoverability impacts the phonetic implementation of geminates and likely does so whether these are true or fake.

2aSC8. Articulatory comparison of Tamil liquids and stops using real-time magnetic resonance imaging. Michael Proctor (Yale Univ., New Haven, CT 06520), Louis Goldstein, Dani Byrd, Erik Bresch, and Shri Narayanan (Univ. of Southern California, Los Angeles, CA 90089)

It has been hypothesized that liquid consonants differ from stops in that they exhibit more global control over tongue shapes. This has been demonstrated by ongoing work showing greater vowel context effects in the production of stops than liquids in Spanish and Russian. Languages with rich inventories of coronal consonants, like Tamil, provide an interesting challenge for this view, as the presence of retroflex-dental contrasts among the stops may require substantial global tongue control, and therefore inhibit context effects. To test this, dynamic magnetic resonance imaging (MRI) was used to investigate the midsagittal production of Tamil liquids in intervocalic environments. MRI sequences were acquired at a frame rate of 22.4 $\mathrm{Hz}$. The five liquids of Brahmin Tamil were elicited from three speakers in three different vowel contexts. Nasal stop consonants were also elicited at the same places of articulation to compare the influence of vocalic coarticulation on liquids and stops. Greater coarticulatory influences were observed during the production of nasals than during the production of liquids in the same vowel contexts, suggesting that the production of liquids involves a more active control of dorsal articulation than that used in the production of stops. [Work supported by NIH.]

2aSC9. A study of articulatory kinematics of American English diphthongs. Sungbok Lee and Dani Byrd (Dept. of Linguist., Univ. of Southern California, 3601 Watt Way, GFS-301, Los Angeles, CA 90089)

Diphthongs are acoustically characterized by formant movements between initial and final vocalic segments [cf, Holbook and Fairbanks, J. Speech Hear. Res., (1962); Thomas Gay, J. Acoust. Soc. Am., (1968)]. It has been observed that the initial (onset) and final (offset) portions may not correspond to typical monophthongal vowels and that there exists more acoustic variability in the offset portions than in the onset portions. Spectral transition rates have been found to be different from diphthong to diphthong. In the current study, the articulatory properties of American English diphthongs are investigated in tandem with their acoustic properties. We collected both acoustic and articulatory data of five diphthongs and seven monophthongs in [b-t] context embedded in a carrier sentence using electromagnetic articulography. The data were recorded from two male and one female subjects, and each diphthong was repeated five times. The main analysis focus is on the kinematic characteristics of the three different tongue sensors 
(tongue tip, tongue dorsum, and tongue rear) in each diphthong production and the comparisons to monophthongs that are close to either the onset portion or the offset portion of the given diphthong. [Work supported by NIH.]

2aSC10. Revisiting the role of the sublingual cavity in the /s/-/S/ distinction. Christine H. Shadle, Michael I. Proctor, Khalil Iskarous (Haskins Labs., 300 George St., New Haven, CT 06511, shadle@haskins.yale.edu), and Maria A. Berezina (MIT-SHBT, Cambridge, MA 02139)

The sublingual cavity (SC) has been shown to play an important role in shaping the spectra of consonants, potentially distinguishing /s/ from / $/$ / This work reports on acoustic-articulatory experiments using MRI and mechanical modeling designed to investigate the details of how the SC acts as an acoustic filter and whether it also affects the aeroacoustic source in fricative production. Five American English subjects were imaged while producing fricatives in various vowel contexts. Two subjects that show opposite patterns were studied in detail. In M1, the /s/-// distinction is mainly carried by $\mathrm{SC}$ presence and lip protrusion in $/ \mathrm{J} /$ versus absence in /s/, resulting in marked spectral differences. M2 exhibits significant variability in SC shape and size, and tongue and lip position, in both fricatives; the acoustic spectra likewise vary. Mechanical model experiments show that the noise generation changes as the sublingual cavity is filled in. The main resonance frequency does not necessarily change. It seems thus that the sublingual cavity is just one of many articulatory variables available to the speaker and may not be the principal cause of observed acoustic differences in all subjects. [Work supported by NIH NIDCD DC 006705 to Haskins, NIH NIDCD T32 DC00038 to MIT-SHBT.]

2aSC11. End-state comfort governs kinematic variation in English flap/ tap sequences. Donald Derrick and Bryan Gick (Dept. of Linguist., Univ. of British Columbia, Totem Field Studios, 2613 W. Mall, Vancouver, BC, V6T 1Z4, Canada)

Derrick \& Gick [Can. Acoust. 36(3), 162 (2008)] found that North American English flap/tap singletons are produced using up to four phonetically conditioned kinematic variants (up-flaps, down-flaps, postalveolar taps, and alveolar taps), dependent on the position of the tongue tip in neighboring rhotic and nonrhotic vowels. Our pilot work has shown that sequences of multiple flap/tap variants are highly mechanically interdependent (e.g., subjects never produce two up-flaps or two down-flaps in adjacent syllables). The present study tests flap/tap sequences for compliance with “end-state comfort" [Rosenbaum et al., Acta Psychologica 94, 59 (1996)], as evidence for look-ahead planning. Twelve subjects' tongue positions were measured during production of English words and word pairs with one- and two-flap sequences. Results show that sequences of flaps end with a preferred tongue tip position. For example, for 11 subjects, the /d/ of "edit" is realized as an alveolar tap or down-flap in "edit the," but an up-flap in "edit a," both facilitating ending the sequence with a low tongue position. A general avoidance of a sequence-final up-flap was also observed, such that /d/ of "edify" is an alveolar-tap or down-flap, while, for eight subjects, "editor" shows an up-flap, postalevolar tap sequence. [Work supported by NSERC.]

2aSC12. An articulatory study of lexicalized and epenthetic schwa using real time magnetic resonance imaging. Adam Lammert (Dept. of Comp. Sci., Univ. Southern California, 941 W. 37th P1. Los Angeles, CA 90089), Erik Bresch, (Univ. Southern California, Los Angeles, CA 90089), Dani Byrd, Louis Goldstein, (Univ. Southern California, Los Angeles, CA 90089), and Shrikanth Narayanan (Univ. Southern California, Los Angeles, CA 90089)

Debate exists about the nature of schwa in terms of articulatory planning and execution. It is commonly believed that schwa lacks a specific constriction target. Another view is that the constriction target for schwa is the mean of the language's targeted vowels. Some have argued that both lines of thinking are correct [I. Smorodinsky, J. Acoust. Soc. Am. 109, 2446 (2001)]. Specifically, epenthetic schwas (e.g., needed) may indeed be targetless, while lexical schwas (e.g., panda) have a target. To investigate this, we collected midsagittal realtime 1 MR images [S. Narayanan et al., J. Acoust. Soc. Am. 115, 1771 (2004)] from two native English speakers. The speakers read sentences aloud which contained, variously, lexical or epenthetic schwas in multiple contexts. Images of the various schwas were extracted from the MR data and a profile of the vocal tract was fit to the image. If both targeted and targetless schwas exist, then they should be evident by examining the vocal tract shape in these images. Lexical schwas, if they are targeted, should have relatively low variability as compared to epenthetic schwas. Conversely, the vocal tract shape during epenthetic schwas should be highly correlated with the surrounding vowel context. [Work supported by NIH.]

2aSC13. Evidence of an articulatory saturation effect in the production of /s/ in American English. Joseph S. Perkell, Melanie L. Matthies, and Mark Tiede (Speech Commun. Group, Res. Lab. of Electronis, MIT, Rm. 36-591, 50 Vassar St., Cambridge, MA 02139.)

Previous work [JSLHR, 47, 1259-1269 (2004)] demonstrated that speakers of American English who consistently activated a contact sensor on the lower alveolar ridge with the tongue tip during /s/ but not /sh/ tended to produce greater sibilant contrast than speakers who did not consistently show this contact difference. This result is compatible with the existence of a sublingual cavity during /sh/ that is eliminated by the tongue-ridge contact for $/ \mathrm{s} /$, which creates an abrupt decrease in volume and resulting rapid increase in resonant frequency of the cavity anterior to the sibilant constriction. This study uses data from articulatory movements (EMMA), contact of the tongue tip with the alveolar ridge, and analysis of the acoustic signal to investigate a hypothesized articulatory saturation effect: abrupt articulatory and acoustic changes result from continuous forward movement of the tongue, which does not have to be controlled precisely in stopping at /s/ onset. In tentative support of this hypothesis, preliminary observations from productions of continuous /sh/-to-/s/ transitions show movements of tongue-blade points, which slow but do not stop immediately following articulatory contact and/or a rapid rise in sibilant spectral mean. Additional tests of the hypothesis and corresponding results will be reported. [Work supported by NIDCD.]

2aSC14. Relation of vocal tract constriction location to identification of voiced stop consonants. Brad H. Story and Kate Bunton (Dept of Speech, \& Lang., Hearing Sci., Univ. of Arizona, P.O. Box 210071, Tucson, AZ $85721)$

Consonant identification experiments are typically based on stimuli produced with a formant synthesizer where a particular acoustic characteristic is varied along some continuum of values (e.g., onset frequency of F2). In this experiment, however, vowel-consonant-vowel (VCV) stimuli were generated with a synthesizer based on a kinematic model of the vocal tract shape [B. H. Story, JASA, 117(5), 3231-3254 (2005)] where the acoustic characteristics result from the time-varying tract configuration. Each VCV was produced by superimposing a constriction at a particular location within the vocal tract on an underlying vowel-vowel sequence. Five different vowel-vowel sequences were produced, and for each, a constriction was imposed at 20 consecutive locations extending from the lips to approximately the velum. VCVs were presented in random order, ten times each, to ten listeners, using a forced choice paradigm where only the voiced English consonants /b, d, g/ were available. Listener responses indicated that the relation of formant frequencies near the consonant, to those of the preceding or succeeding vowel can provide reasonably accurate predictions of regions within the vocal tract that correspond to specific consonants. It was also revealed that the location and length of each region is dependent on the vowel. [Research supported by NIH R01-DC04789.]

2aSC15. Phonetic properties of syllabic consonants in Slovak. Marianne Pouplier (Inst. of Phonet. , Ludwig Maximilians Univ., Munich, Schellingstr 3/ii, 80799 Munich, Germany, pouplier@phonetik.uni-muenchen.de) and Štefan Beňuš (Constantine the Philosopher Univ., 94974 Nitra, Slovakia)

Studies on the articulatory correlates of syllable structure have shown that onset and coda consonants differ systematically in the timing and amplitude of their movements, and their temporal coordination with the vocalic syllable nucleus. Little is known, however, about consonants functioning as syllable nuclei. We present EMA data on Slovak, a language that allows the liquids /1/ and /r/ to be syllabic nuclei of CCC syllables. Phonologically, syllabic consonants behave like vocalic nuclei. We investigate the kinematic properties of syllabic consonants, and potential differences between vocalic and consonantal nuclei in their patterns of coordination with onsets and codas. Our data indicate that liquids in onset clusters are shorter than singletons (e.g., /r/ in "mrak" < "rak"), yet syllabic consonants (/r/ in "mrk") pattern durationally with singletons. Generally, Slovak onset clusters show little 
or no overlap, which may result in an open transition schwa. For $\mathrm{C} 1 \mathrm{C} 2 \mathrm{C} 3$ syllables, C1-C2 show more overlap compared to an onset cluster, yet open transition schwas can still be observed ("m^rk"). C2-C3 overlap less than $\mathrm{C} 1-\mathrm{C} 2$ in terms of movement onset, yet C1-C2 show a bigger plateau lag. These data contribute to our knowledge about the phonetic nature of syllabic consonants and articulatory correlates of syllable structure.

2aSC16. Rate conditioned variability in Western Andalusian Spanish aspiration. Benjamin Parrell (Dept. of Linguist., Univ. of Southern California, Grace Ford Salvatori 301, Los Angeles, CA 90089, parrell@usc.edu)

Western Andalusian Spanish normally shows aspiration of /s/ in coda position, but post-, rather than pre-, closure aspiration in $/ \mathrm{s} /+/ \mathrm{p}, \mathrm{t}, \mathrm{k} /$ sequences. This has been hypothesized to be the result of gestural reorganization, with pre-closure aspiration the result of anti-phase coordination and post-closure the result of in-phase [Torreira, 9th HLS, 2007]. This experiment explores this hypothesis by leveraging the fact that fast speech can lead to instability in gestural organization and even a spontaneous switch from anti- to in-phase coordination [Turvey, Am. Psych., 1990]. Subjects produced words with the target $/ \mathrm{s} /+/ \mathrm{p}, \mathrm{t}, \mathrm{k} /$ sequences in time with an acoustic metronome that steadily increased in rate. Results show three patterns of production: consistent preaspiration, consistent postaspiration, and a transition from pre- to post-aspiration as rate increased, corresponding to antiphase, in-phase, and an anti- to in-phase transition, respectively. Excluding the trials with consistent postaspiration, which would not be expected to transition given their stable in-phase coordination, postaspiration increases as rate increases. If there were no change in gestural coupling, we would expect the opposite pattern: as rate increases, VOT should decrease. The result thus supports the original hypothesis that postaspiration in these sequences is the result of gestural re-phasing.

2aSC17. A production study on phonologization of /u/-fronting in alveolar context. Reiko Kataoka (Dept. of Linguist., Univ. of California, Berkeley, 1203 Dwinelle Hall, Berkeley, CA 94720-2650)

Recently many researchers modeled sound change as the result of phonologization [Hyman (1976)], a process whereby coarticulatory perturbation of a speech sound becomes intended properties of the sound. Although phonologization is a useful concept, diagnostic criteria have yet to be proposed. By using / $\mathrm{u}$ /-fronting in alveolar context in American English as a case study, this paper demonstrates how production data of various speech rates may be used to differentiate phonologized speech variations from mechanically caused variations. English speakers produced $/ \mathrm{CuC} /$ forms where the place of both Cs was either alveolar (text context) or other (control context), in fast, medium, and slow speech rates. [Lindblom (1963)] demonstrated that the magnitude of coarticulatory influence on the vowel reduces as the vowel's duration increases. Thus, if the fronting of the vowel in alveolar context persists regardless of its duration, then this would constitute as evidence for phonologization. The results from a study with 30 speakers show that the vowels had significantly higher mean F2 values in alveolar contexts than in bilabial or zero contexts even in slow speech rate, suggesting that speakers have distinct production goals for $/ \mathrm{u} / \mathrm{s}$ in different contexts, or $/ \mathrm{u} /-$ fronting has been phonologized.

2aSC18. Acoustic cues to stop coda voicing contrasts in 1 to 2-year-olds' American English. Katherine Demuth (Dept. of Cognit. and Linguistic Sci., Brown Univ., 190 Thayer St., Providence, RI 02912), Stefanie ShattuckHufnagel (Massachusetts Inst. of Technol., Cambridge, MA 12139), Jae Yung Song (Brown Univ., Providence, RI 02912 and MIT, Cambridge, MA 12139), Karen Evans, Jeremy Kuhn, and Miranda Sinnott-Armstrong (Brown Univ., Providence, RI 02912)

One limitation in understanding the mechanisms underlying children's variable productions has been the reliance on perception-based segmental transcription. This raises the possibility that children may make covert, acoustic distinctions that are not perceived by the transcriber. ShattuckHufnagel et al. [S. Shattuck-Hufnagel, K. Demuth, H. Hanson \& S. Stevens, submitted] investigated this issue employing Stevens' [K. N. Stevens, J. Acoust. Soc. Am. 111, 1872-1891 (2002)] feature-based acoustic model of perception/production. They found that 2,6-3,6-year-olds exhibited systematic acoustic cues to coda voicing contrasts (e.g., dog versus duck): voicebar was more likely to precede voiced codas, whereas vowel glottalization was more likely to precede voiceless codas. The present study extended this investigation to velar codas in three 1,6-2,6-year-olds and their mothers, showing similar results: both exhibited more use of voice-bar before voiced compared to voiceless codas. For mothers, the duration of voice-bar was also longer for voiced codas, and children showed a trend in this direction. However, only mothers showed a significantly higher use of vowel glottalization before voiceless codas. Thus, although younger children also produce some acoustic cues to voicing distinctions, others take time to become adultlike. Physiological and environmental factors are discussed to explain these results. [Work supported by R01 HD057606.]

2aSC19. Segmentation of speech based on perceptual equivalence: Case of alveolar trill. Diana Stojanovic (Dept. of Linguist., Univ. of Hawaii, 1890 East-west Rd., Moore Hall 569, Honolulu, HI 96822)

Turk et al. (2006) proposed a set of rules for segmentation in durational studies. R-sounds were not included in the recommended class due to their variable realizations. The authors suggested segmentation of tap variants was straightforward but that of approximants prohibitively difficult. In this paper, we discuss the issue of segmentation of the alveolar trill in Serbian. Three native speakers read a set of minimal pair phrases in which consonants $/ \mathrm{d} /, / \mathrm{n} /$, and /r/ occurred intervocalically. Phrases were matched for the position and type of pitch-accent. The four-syllable phrases produced by each speaker were judged by the native listeners as being rhythmically equivalent. When $/ r /$ is segmented to include only the closure, vowels matched for quality, position, and accent type are significantly longer before $/ \mathrm{r} /$ than before $/ \mathrm{d} /$ or $/ \mathrm{n} /$. The three consonants are all voiced thus compensatory lengthening is not likely to affect the length of the preceding vowel. Based on perceived rhythmic equivalence of the examined phrases, we formulate a segmentation rule that places the start of /r/ during the vocalic portion preceding the closure. This strategy equalizes measured durations of the preceding vowels and additionally enables us to treat $/ \mathrm{r} /$ equivalently in all environments.

2aSC20. Non-native listener's linguistic background and use of contextual cues. Lu-Feng Shi (Dept. of Commun. Sci. and Disord., Long Island Univ.-Brooklyn Campus, One Univ. Plaza, Brooklyn, NY 11201, lu .shi@liu.edu)

Non-native listeners have more difficulty perceiving acoustically degraded English speech than native listeners. In addition, performance in non-native listeners varies greatly depending on many linguistic factors. Recognizing the importance of these factors, the current study assessed how 30 non-native listeners utilized contextual information on the reverberant speech-perception-in-noise test [Sandridge et al., AAS (2005)] based on their linguistic profiles, obtained through the Language Experience and Profile Questionnaire [V. Marian et al., JSLHR 50, 940-967 (2007)]. Specifically, these SPIN sentences (high versus low context) were processed in two levels of multi-talker babble (signal-to-noise ratio $=+6$ versus $0 \mathrm{~dB}$ ) and two levels of reverberation (reverberation time $=1.2$ vs $3.6 \mathrm{~s}$ ). Significant factors in language dominance, proficiency, learning, and use were identified using correlation and multiple regression procedures. Most strikingly, factors related to reading in English (age of acquisition, age of fluency, and preference) emerged as the strongest predictors of performance regardless of the main effect (noise, reverberation, or context). Most variance in performance could be accounted for by these reading factors and two additional factors related to speaking in English (proficiency and accent).

2aSC21. Acoustic analysis of the tones and tone sandhi in Hakka Chinese. Eric Zee and Wai-Sum Lee (Dept. of CTL, City Univ. of Hong Kong, 83 Tat Chee Aven., Kowloon, Hong Kong, eric.zee@cityu.edu.hk)

This paper analyzes F0 contours of (i) the six citations tones of Hakka Chinese which consist of four long tones that occur on open syllables and closed syllables with a nasal and two short tones on checked syllables and (ii) tone contours on 36 bisyllabic compounds, representing possible permutations of the six citation tones, and 120 commonly used trisyllabic compounds. Based on the F0 data, (i) tone values are assigned to the citation tones and (ii) tone patterns on the bisyllabic and trisyllabic compounds are defined. Tone sandhi rules are formulated, summing up the phonological changes in the citation tones when juxtaposed. Phonetic tonal coarticulations are also examined. The F0 data show that the citation tones may be represented in terms of tone letter as [53 3133 11] (long) and [ 5 5 32] (short). Major tone sandhis include [33] becoming [35] and [53] becoming 
[55] before [11], [31], or [53]; and before another [31], [31] becomes [33]. Process such as [ $\left.\begin{array}{lllll}53 & 53 & 53 & 53\end{array}\right]$ becoming [ $\left.\begin{array}{llll}5 & 5 & 5 & 53\end{array}\right]$ illustrates simultaneous application of tone sandhi to only tones on the non-compound-final syllables, and [ [ $\left.\begin{array}{llllll}5 & 33 & 11 & 53 & 31\end{array}\right]$ do not undergo tone sandhi before [5] or [33].

2aSC22. Effect of tone on consonant + vowel interaction. Augustine Agwuele (Dept. of Anthropology, Texas State Univ. San Marcos, 601 University Dr., San Marcos, TX 78666)

Examining consonant + vowel coarticulation for American English [Agwuele et al., 16th ICPhS, 1, 617-620 (2007) and Lindblom et al., J. Acoust. Soc. Am. 121, 3802-3813 (2007)], the effect of speech tempo and emphatic stress on F2onset and F2mid of the following vowel were quantified and dissociated from the underlying traditional coupling of consonant and vowel. Thus, these two prosodic factors were shown to pose additional confound to $\mathrm{CV}$ coarticulation. Adopting the approach of these works, this present study examines VCV sequences in Yoruba, a tonal language, in order to determine whether lexical tone produces as additional confounding effect to the interaction between consonant and tone bearing vowel that could be shown to be separate from the traditionally expected CV bonding. The study shows that the changing pattern of tone configuration in VCV sequences, e.g., from VMCVM [M=midtone $]$ to $\mathrm{VLCVL}[\mathrm{L}=$ low tone $]$ produces differing consonant + vowel interaction. The study provides acoustic evidence for a possible independence of the "source" from the "filter," i.e., the resonances of the vocal tract [Fant, G. Acoustic Theory of Speech Production (1960)].

2aSC23. The production and perception of Mon voice registers. Arthur S. Abramson, Gordon Ramsay (Haskins Labs., 300 George St., Ste. 900, New Haven, CT 06511, arthur.abramson@uconn.edu), and Theraphan Luangthongkum (Chulalongkorn Univ., Bangkok 10330, Thailand)

As a continuation of our work on voice registers [Abramson et al., Phonetica 61, 147-171 (2004); Abramson et al., Phonetica 64, 80-104 (2007)], another language, Mon, has been examined. A voice register on a syllable is a bundle of phonetic properties among which one, usually phonation type, is paramount. Fieldwork, with support from acoustics [Thongkum, in Bamroongraks et al., (eds.), International Symposium on Language and Linguistics, pp. 22-50 (1998)], has yielded primarily modal voice and higher pitch for one register and breathy voice and lower pitch for the other. Our acoustic analysis has confirmed the significance of F0 height as well as that of the intensity ratios of higher harmonics and the first harmonic. The greater attenuation of the higher spectrum for breathy voice is taken as a consequence of a larger open quotient of the glottal cycle. For validation, electroglottography of the native speakers used for our acoustic analysis has been done. A control test of the robustness of the distinction in natural speech has been run along with one with synthetic speech for the efficacy of incremental changes along three dimensions, F0, spectral slope, and turbulence mixed with glottal pulsing. Final data will be shown.

2aSC24. Tone space reduction in Mandarin Chinese. Jeffrey Berry (Dept. of Linguist., Univ. of Arizona, Douglass Bldg., Rm. 200E, Tucson, AZ 85721)

Work on clear versus conversational speech has shown that speakers expand the vowel space in clear speech, which maximizes the perceptual contrast, making it easier for listeners to parse the speech signal. This study investigates how the realization of lexical tones changes for different speech tasks, and whether it is sensitive to the number of repetitions of the same word in a conversation. Native Mandarin speakers participated in a map task in which they were instructed to explain the route on the map to a partner. The first and last tokens of each target word were extracted from the dialogues and compared to the same word read from a list. Pitch and duration were normalized and quadratic curve fitting was performed on the test items. Results of statistical analyses show that contour tones from later occurrences of the target words have a smaller slope, are more linear, and tend to have pitch values closer to the overall average pitch of the speaker. In other words, all tones are realized closer to a midlevel tone when they have been previously mentioned in the conversation. In this sense, there is a contraction of the tone space for later tokens.

2aSC25. Contextual tonal variation in level tone languages. Kristine $M$ Yu (Dept. of Linguist., Univ. of California, Los Angeles, 3125 Campbell Hall, Los Angeles, CA 90095, krisyu@ @umnet.ucla.edu)
While contextual tonal variation in languages with contour tones has been well studied [Han and Kim (1974); Gandour (1992); Xu (1994)], contextual tonal variation has been less well-studied in level tone languages [Meyers (1976)]. The acoustic variation of tones due to different tonal contexts in Hausa, Bole (with two tones $\mathrm{H}$ and $\mathrm{L}$ ) and Yoruba (with three tones $\mathrm{H}, \mathrm{M}$, and $\mathrm{L}$ ) will be investigated. The description of cross-linguistic contextual tonal variation is essential for understanding the task that infants are undertaking in acquiring tonal categories in the face of variance in tonal realization. The focus of the investigation will be how the acoustic variation compares to that in languages with contour tones and if there are general patterns across languages or language-specific differences in variation.

2aSC26. Perception of Mandarin Chinese tone 2/tone 3 and the role of creaky voice. Rui Cao and Ratree Wayland (Linguist., Univ. of Florida, 4131 Turlington Hall, PO Box 115454, Gainesville, FL 32611-5454, ruicao @ufl.edu)

Previous research has established that among the four tones of Mandarin Chinese, tone 2 and tone 3 are very similar in their pitch contour shapes and are, thus, difficult to distinguish by native and nonnative listeners. On the other hand, creaky voice has been reported to be associated with tone 3 production among native speakers. However, the role of creaky voice in the perception of tone 3 has yet to be studied systematically. This study aims to investigate (1) the extent to which creaky voice is present in tone 3 production by native speakers; (2) the perceptual boundary between tone 2 and tone 3 among native and non-native speakers; and (3) the effect of creaky phonation on this perceptual boundary. Thirty native Chinese speakers and 30 native speakers of American English who are learning Chinese will participate in this study. Tone stimuli with and without creaky voice will be presented auditorily for tonal identification. The data obtained will be analyzed to examine the effect of creaky voice in the identification of tone 3 among these two groups of speakers. In addition, the native production of tone 3 will be examined for the presence of creaky voice.

2aSC27. Perceptional experiment on Mandarin tones by Mandarin, Spanish, and Cantonese speakers. Yi-Chu Ke (Dept. of Foreign Lang. and Linguist., Natl. Chiao Tung Univ., 1001 Univ. Rd., Hsinchu, Taiwan 300, ROC), Mu-Ling Teng, Chu-ting Chen, Lai-Iok Ip, Bo-hong Lu (Natl. Chiao Tung Univ., Taiwan 300, ROC), Shih-wen Chen (Natl. Tsing Hua Univ., Hsinchu, Taiwan 30013, ROC), Hsiu-Min Yu (Chung Hua Univ., Hsinchu, Taiwan 300, ROC), and Ho-hsien Pan (Natl. Chiao Tung Univ., Taiwan 300, ROC)

This paper discussed perception of three contrastive Mandarin tones: high tone (Tone1), rising tone (Tone2), and falling tone (Tone4). Based on the previous study by Halle (2004), in which tone (Mandarin) and non-tone (French) speakers joined, our perceptional experiment employed both identification and discrimination paradigm, and categorical result of tones was expected. We recorded two Mandarin words "tu" and "wa" in those three contrastive tones, and these six words were manipulated into same duration. We then synthesized the F0 of Tone1 to Tone2, Tone1 to Tone4, and Tone2 to Tone4 in seven steps. Subjects were separated in accordance to their mother tongues, namely Mandarin, Spanish, and Cantonese. Categorical perception is obvious in Mandarin group. Cantonese speakers revealed a similar categorical pattern to Mandarin speakers, while Spanish speakers showed no categorical pattern. On the whole, Mandarin speakers had an earlier dropdown on second or third step over each pair of tone stimuli.

2aSC28. Fundamental frequency in English and Mandarin. Patricia Keating (Phonet. Lab., Dept. of Linguist., Univ. of California at Los Angeles, Los Angeles, CA 90095-1543, keating@humnet.ucla.edu)

It has been suggested that Mandarin, as a tone language, uses a larger F0 range than English. This study addresses two questions: (1) Do English and Mandarin F0 profiles differ? (2) How does the type of voice sample which is measured affect this F0 characterization and language comparison? Native speakers of English or Mandarin produced a variety of speech samples intended to elicit a wide range of F0s. Maximum phonation frequency range was measured from pitch sweeps, and speaking F0 was measured from readings of the Rainbow passage, isolated words with various intonations, and a dramatic story. Cepstral pitchtracking was used to log the entire F0 contour for each utterance, and various measures were computed. Preliminary results suggest, first, that the maximum phonation frequency ranges are the same 
for the two languages; second, that in reading Mandarin speakers use a higher mean F0, but at the same time English speakers use higher maximum F0 and wider range of F0; and third, that these differences do not depend on the speech samples being compared. [Work supported by NSF.]

2aSC29. Breathing behavior in relation to prosody and linguistic structure in spontaneous speech. Peter J. Watson (Dept. of Speech-Lang.Hearing Sci., Univ. of Minnesota, 164 Pillsbury Dr. SE, Minneapolis, MN $55455)$

Hird and Kirsner [K. Hird and K. Kirsner, Brain Lang., 80, 536-555 (2002)] reported that F0 is reset at higher values at the beginning of grammatically complete sentences during spontaneous speech. Hird and Kirsner reported that inspirations taken at the beginning of complete sentences "appeared" to be deep, but they did not make physiological measures of breathing behavior. [Watson, et al. J. Acoust. Soc. Am., 113, 2812-2819 (2003)] found that when sentences were read aloud at higher values within the vital capacity they were initiated at higher F0s; however, these measures were not made during spontaneous speech and grammatical structure was not varied. For this study, we measured breathing behavior using Respitrace while three women and one man were interviewed. Posthoc, breath groups were grammatically organized according to the method used by Hird and Kirsner. On average, breath groups that comprised complete sentences were initiated from higher lung volumes and F0 was reset at higher levels at the beginning of these breath groups. Results will be discussed in relation to the interaction of linguistic structure and breathing behavior.

2aSC30. How do ordinary listeners perceive prosodic prominence? Syntagmatic versus paradigmatic comparison. Yoonsook Mo, Jennifer Cole (Dept. of Linguist., Univ. of Illinois, Urbana-Champaign, $405 \mathrm{~N}$. Mathews Ave., Urbana, IL 61801), and Mark Hasegawa-Johnson (Univ. of Illinois, Urbana-Illinois, Urbana, IL 61801)

In comprehending speech, listeners are sensitive to the acoustic variation encoding the prosodic structures that mark phrasing and prominence. The present study, based on prosody transcriptions of untrained listeners (74 monolingual American English speakers), tests which acoustic feature or feature combinations cue prosodic prominence, and specifically, whether ordinary listeners perceive prosodic prominence based on changes in acoustic measures in the local context (syntagmatic comparison) or based on the value of an acoustic measure relative to the distribution of that measure across all instances of the specific phoneme in the listener's experience (paradigmatic comparison). Subjects listened to 36 short excerpts (11-25 s) of spontaneous speech from the Buckeye Corpus, and marked prominent words on a transcript. After evaluating intertranscriber agreement rates, acoustic measures (duration, intensity, subband intensities, F0 max, and formants) were extracted from stressed vowels and normalized within two domains (syntagmatic/paradigmatic). The results show that all the acoustic measures are correlated with perceived prominence and combinations of the acoustic measures account for the variability in listeners' perception of prominence. Moreover, syntagmatically normalized acoustic measures explain more of the variability, indicating that ordinary listeners perceive prosodic prominence based on local changes in acoustic measures and especially in changes in overall intensity.

2aSC31. Computational modeling of juncture strength using articulatory synthesis of prosodic gestures. Sungbok Lee, Benjamin Parrell, and Dani Byrd (Dept. of Linguist., Univ. of Southern California, 3601 Watt Way, GFS-301, Los Angeles, CA 90089)

Prosodic boundary gestures or "pi-gestures" (Byrd and Saltzman, J. Phonetics, 2003) have been introduced to model the local slowing or lengthening of articulatory gestures in the vicinity of phrase boundaries. Computational modeling of articulatory dynamics is an important tool in assessing the predicted effects of pi-gestures of varying boundary strength on constriction gestures in varying contexts. We simulate pi-gestures within the TaDA task dynamics computational model [Nam and Kim, JASA, 116, 172-185 (2004)] and examine how functional data analysis can provide a tool for connecting articulatory lengthening with underlying pi-gesture activation strength. Specifically, the model is applied to the articulatory synthesis of two sequences: [CV\#CV] and [CVC\#CV], where $\mathrm{C}$ is bilabial or alveolar. The pi-gesture's midpoint is coordinated synchronously with the midpoint of following consonant's constriction gesture, and pi-gesture activation strength and duration are manipulated. Results indicate that pi-gesture activation strength has a much stronger effect on slowing than its duration. The slowing effect is asymmetrical, skewed earlier than the midpoint in the pigesture interval. After removing linear-time slowing effect (i.e., after length normalization), the slowing effect is slightly stronger in $[\mathrm{CV \# CV}]$ than in [CVC\#CV]. The strength of pi-gesture also affects spatial articulatory characteristics depending on constriction location and sequential context. [Work supported by $\mathrm{NIH}$.]

2aSC32. Comparing contrastively focused and clear speech productions of color terms. Melissa A. Redford (Dept. of Linguist., Univ. of Oregon, 1451 Onyx St., Eugene, OR 97403-1290, redford@uoregon.edu), Adriano Vilela Barbosa, and Eric Vatikiotis-Bateson (Univ. of British Columbia, Vancouver, BC V6T 1Z4, Canada)

Words that are prosodically marked as focused in English are phonetically similar to clear speech words. Just as clear speech words are longer and better articulated (hyperarticulated) compared with matched casual speech words, prosodically focused words are longer and hyperarticulated compared to matched, unemphasized words. These similarities suggest that the implementational difference between prosodic focus and clear speech is merely one of scope: the same parameters are adjusted to either highlight a single element in an utterance (focus marking) or all the elements in an utterance (clear speech). To explore this idea we investigated the acoustics and jaw kinematics of producing color terms in either a casual style, a clear speech style, or with contrastive focus. Preliminary acoustic results indicate that clear speech lengthening is greater than focus-marking lengthening in some speakers. These same speakers also vary fo more when contrastively focusing color terms than when producing them clearly, though this effect interacts with phrase position. Focused and clearly produced color terms are similarly hyperarticulated relative to casually produced terms. The results suggest that most speakers have different parameter settings for prosodic highlighting than for clear speech.

2aSC33. Acoustic correlates of rhythm in Western Cham. Kaori Ueki (Dept. of Linguist., Univ. of Hawaii, 1890 East-West Rd., Honolulu, HI 96822,kueki@hawaii.edu)

Chamic languages, which are part of the Austronesian language family, are generally known to bear lexical stress on final syllables [Thurgood, (2005)] but no instrumental studies of its prosodic features have been carried out. The current study seeks quantitative results to back up these impressionistic descriptions, and to examine how this language compares against the prosodic typology in June 2005. Syllable rimes of Western Cham, an understudied Chamic language, were measured for duration with respect to position of syllable within a phrase (initial, medial, final). The study shows that durations for final syllables were significantly longer, and that unstressed syllables are reduced in all positions. Preliminary results show that durations and pitch excursions of syllable rimes do not differ markedly with respect to phrasal position. Interactions of pitch and duration at lexical and postlexical levels will also be discussed.

2aSC34. Boundary- and prominence-related lengthening and their interaction. Argyro Katsika (Dept. of Linguist., Yale Univ., 370 Temple St., New Haven, CT 06520, argyro.katsika@yale.edu)

The durational effects of prosodic boundaries and prosodic prominence are well known, but their interaction is less well-understood. Recent studies in English [Turk and Shattuck-Hufnagel (2007); Byrd and Riggs (2008)] indicate that the two effects might be interdependent. Two acoustic experiments are presented, examining the role of boundary- and prominencerelated lengthening and their interaction in Greek. The first experiment explores the effects of boundary-adjacent lengthening (conditions: no boundary, intermediate phrase, intonational phrase) and prominence (on the first, second, and third syllable away from the boundary). The second experiment examines these same effects, but post-boundary. Data from eight speakers were collected. The results from five speakers are presented. Results show that pre-boundary, both prominence and boundary have an effect on segment duration. Two prosodic levels (the level of word and one higher level) are distinguished. Furthermore, for two speakers, there is an interaction effect, such that three prosodic levels are distinguished when the prominent syllable is boundary-adjacent. The post-boundary condition shows less systematic results, with different speakers exhibiting different patterns. 
These results suggest that prosodic effects are speaker dependent, more stable pre-boundary, and that prominence and boundaries interact, increasing prosodic boundary marking. Implications for models of prosodic structure are discussed.

2aSC35. Real-time MRI tracking of articulation during grammatical and ungrammatical pauses in speech. Vikram Ramanarayanan (Speech Anal. and Interpretation Lab., Dept. of Elec. Eng., RTH 320, 3710 McClintock St., Univ. of Southern California, Los Angeles, CA 90089, vramanar@usc.edu), Erik Bresch, Dani Byrd, Louis Goldstein, and Shrikanth S. Narayanan (Univ. of Southern California, Los Angeles, CA 90089)

Grammatical pauses in speech generally occur at a clause boundary, presumably due to parsing and planning; however, pausing can occur at grammatically inappropriate locations when planning, production, and/or lexical access processes are disrupted. Real-time MRI of spontaneous speech production (responses to queries like "tell me about your family," etc.) was used for seven subjects to examine the articulatory manifestations of grammatical and ungrammatical pauses (manually classified as such by two experimenters depending on the presence/absence of a clausal juncture). Measures quantifying the speed of articulators were developed and applied during these pauses as well as their immediate neighborhoods. Results indicate a consistently higher articulatory speed and spatial range for grammatical compared to ungrammatical pauses, and an appreciable drop in speed for grammatical pauses relative to their neighborhoods, suggesting that higher-level cognitive mechanisms are at work in planning grammatical pauses. On the other hand, ungrammatical pauses show only a slight decrease in articulator speed at the pause but are followed by a spurt in speed immediately after, suggesting that the planning mechanism has been reengaged only at that point, giving an indication of how much time it takes to "recover" from the perturbation of the linguistic structural integrity of the utterance. [Work supported by NIH.]

2aSC36. A corpus search methodology for focus realization. Jonathan Howell and Mats Rooth (Dept. of Linguist., Cornell Univ., 203 Morrill Hall, Ithaca, NY 14853-4701, jah238@ cornell.edu)

We describe a methodology for investigating the semantic-grammatical conditioning and phonetic realization of contrastive intonation using a web harvest of particular word strings followed by grammatical and acoustic analysis. A commercial audio web search engine using speech recognition retrieved 179 MP3 files purportedly containing a token of the string "than I did." In this comparative clause fragment, contrastive focus commonly falls on the subject (she did more than $\mathrm{I}_{F}$ did), on "did" (I wish I had done more than $\mathrm{I}_{\operatorname{did}_{F}}$ ), or following (I said more now than I did before ${ }_{F}$ ). The 96 true tokens of "than I did" were classified into the categories "subject," "did," and "following" by grammatical and semantic criteria. For each token, 5 segment intervals were hand-annotated and more than 300 acoustic parameters extracted using a Praat script. SVM machine learning classifiers were trained that identify focus classes by acoustic criteria. On a 10-fold crossvalidation test, the classifier achieves $90.2 \%$ accuracy in discriminating the dominant "subject" and "following" classes. In a listening task, human subjects achieved comparable accuracy of $90.3^{\prime}$ given only the acoustic target "than I did." Stepwise logistic regression revealed measures of duration, $f 0$, intensity, formants, and formant bandwidths among the significant factors.

2aSC37. Prosodic planning: Local and distant effects of phrase length on pause duration. Jelena Krivokapic (Dept. of Linguist., Yale Univ., 370 Temple St., Rm. 302, New Haven, CT 06511, jelena.krivokapic@yale.edu)

Prosodic boundaries, as manifested in pause duration, are known to be influenced by structural properties of the phrases immediately preceding and following the boundary, such as their syntactic structure, prosodic structure, and phonological length. Little is known, however, about how phrases at a distance from a boundary, i.e., not immediately preceding or following it, may affect the durational characteristics of pausing at that boundary. An experiment is presented that investigates the effects on pause duration of prosodic phrases of different length, both immediately at the boundary and farther away from it. The goal is to (a) examine local and distant effects of prosodic phrase length on pause duration and (b) to examine incrementality in production of prosodic structure, i.e., to examine how far ahead speakers plan an utterance, and how prosodic structure affects the planning process. Subjects read 24 English sentences varying along the following parameters: (a) length in syllables (two or four syllables) of the intonational phrase immediately following a target pause and (b) length in syllables of the second, distant, intonational phrase following the pause (10 or 16 syllables long). The synchronous speech paradigm [F. Cummins (2002); E. Zvonik, F. Cummins (2002)] was used to minimize variability in production.

2aSC38. Duration and fundamental frequency patterns in Brazilian Portuguese polysyllabic words. Pablo Arantes and Plinio Barbosa (Dept. of Linguist., Campinas St. Univ., PO Box 6045, Campinas, SP, Brazil 13083-970,parantes@ fastmail.fm)

Brazilian Portuguese polysyllabic words are said to have secondary stress (SS). There is no clear consensus, though, on how SSs are assigned and what the acoustic correlates for SSs are. We analyzed a corpus of read speech in order to gain some insight on those two issues. The corpus' target words have 2 to 4 pre-stressed syllables with primary lexical stress falling either on the last or penultimate syllable. Target words appeared in initial, medial, or final position on carrier sentences. Previous works showed that duration and F0 are the main correlates of stress marking in BP with a marginal contribution of vowel quality and spectral emphasis. Statistical analysis of normalized ( $z$-scored) syllable and vowel-to-vowel duration show no evidence of binary alternation. Duration increases exponentially up to the stressed syllable. A Praat script was used to determine global and local F0 maxima and minima. Lexically stressed syllables have F0 peaks associated with them. There is at most one F0 peak on the pre-stressed syllable chain. Peaks tend to be higher in 4-pre-stressed-syllable words and be absent in 2-pre-stressed-syllable words. Target word position do not have a significant effect on duration contour shape and on F0 peak placement.

2aSC39. Rhythmic structures in content word untterances. Joo-Kyeong Lee (Dept. of English Lang. and Lit., Univ. of Seoul, 90 Jeonnong-dong, Dongdaemun-gu, Seoul, 130-743, Korea) and Sunmi Kang (Korea Univ., Seoul, 136-701, Korea)

This study investigates rhythmic structures in the utterances composed of only content words. Content words are potentially accentable in utterance, and English necessitates a rhythmic alternation between strong and weak beats. What will happen if only content words are sequenced and they are all one-syllable words? A phonetic experiment was conducted to see if a rhythmic pattern is still produced in content word utterances and how it is manifested along the intonational contours. Results showed that merely $7 \%$ of the utterances were produced with every content word accented. Most utterances were rhythmically produced with every two $(38 \%)$ or three $(26 \%)$ words accented, and the other patterns such as a $\mathrm{H}^{*}$ and $\mathrm{L}^{*}$ alternation, two $\mathrm{H}^{*}$ 's intervened with L-, and consecutive downstepped $\mathrm{H}^{*}$ 's were interpreted as conforming the tonal rhythm [J.-K. Lee and S. Kang, Korean J. Speech Sci., 10(2), 303-317 (2003)]. In two-syllable content word utterances, the patterns where two words were unaccented suggest that strong and weak rhythmic beats should be counted with the number of words rather than syllables at the level of utterance. It is proposed that it is an accentual foot as opposed to a lexical foot at the level of lexical word.

2aSC40. The production of English lexical stress by Thai speakers. Jirapat Jangjamras and Ratree Wayland (Program in Linguist., Univ. of Florida, Box 115454, Gainesville, FL 32611-5454, jirapat@ufl.edu)

The major goal of this work is to report acoustic correlates of English lexical stress produced by native and second language learners of American English. Fifteen native Thai speakers and fifteen native English speakers were asked to concatenate two English monosyllabic nonwards into one disyllabic word and to produce stress on either the first or the second of the resulting disyllables. Disyllabic English nonwords were constructed by pairing CVO, CVVO, CVV, CVS, and CVVS monosyllables. It is predicted that Thai speakers' production of English lexical stress will be influenced by Thai prosodic system including Thai tonal distribution rules which are constrained by syllabic structure, the presence of fixed final-stress placement, and the use of duration as the primary cue in L2 English lexical stress production. The acoustic parameters measured included vowel duration, intensity, fundamental frequency, and formant frequencies (F1, F2, F3). The ratio values of these measurements between stressed and unstressed syl- 
lables will be correlated with stress identification scores obtained from native English listener judges to examine the amount of acoustic information required for accurate perception of stress location by native listeners.

2aSC41. Formant frequency development and gender differences in Mandarin-learning children before 5 years of age: A longitudinal analysis. Li-mei Chen (Dept. of Foreign Lang. and Lit., Natl. Cheng Kung Univ., 1 University Rd., Tainan City, Taiwan, leemay@mail.ncku.edu.tw)

This study is designed to investigate the longitudinal developmental process of vocalic production in Mandarin-learning children from birth. This current study focuses on the fifth year and the continuity in the process. Major findings are: (1) From three years and six months, the boy subject showed lower vowel formant values, reflecting gender differences in the development of vocal tract. In addition to three years and six months, there was another stage of decrease of frequency at four years and six months in the boy subject. The trend was especially evident in F2 values. (2) The girl subject used more high front rounded vowels $/ y /$, which is the only lateacquired single vowel. (3) Corner vowels /i, u/ were used more often by the girl subject. (4) Diphthongs continued to show high frequency of occurrence at this stage. (5) The frequency of vowels with nasal endings showed prominent increase at this stage in both genders. However, the girl subject showed more preference in using this vowel category as was found in a previous stage. Gender differences in vowel development found in this stage can be traced in the following longitudinal observation. [This investigation was supported through funds from National Science Council in Taiwan, Republic of China (NSC 96-2411-H-006 -023).]

2aSC42. Effects of speaker gender on voice onset time in Korean stops. Eunjin Oh (Dept. of English Lang. and Lit., Ewha Womans Univ., 11-1 Daehyun-dong, Seodaemun-gu, Seoul 120-750, South Korea, ejoh@ewha.ac $. \mathrm{kr})$

Several studies have shown that the voice onset time (VOT) of English stops varies as a function of speaker sex. The overall trend indicates that females exhibit longer VOT values than males for voiceless stops. The suggested explanations have mainly attributed this difference to physiological or stylistic differences between the sexes. If this is so, we would expect to find identical sex differences in the VOTs of stops in other languages. This study tested 38 native speakers of Korean (19 males and 19 females), who produced fortis, lenis, and aspirated stops in $/ \mathrm{CVn} /$ words. The males tended to exhibit longer VOTs than females for most of the syllables, the greatest differences occurring in aspirated stops. The males also exhibited more distinctive distributions in VOT values than the females in Korean. These results indicate that the physiological or stylistic explanations previously suggested are not tenable since there is no automatic reason why VOT values for voiceless stops are, in general, longer for females in English, but longer for males in Korean. Sociophonetic factors may explain why languages can exhibit opposing trends in gender differences in temporal acoustic properties such as VOTs.

2aSC43. Variation in the phonetic realization of Thai lexical tones across speaking styles. Donruethai Laphasradakul and Ratree Wayland (Program in Linguist., Univ. of Florida, Gainesville, FL 32603)

Thai is a tonal language with five contrastive tones: mid-falling, lowfalling, high-falling, high-rising, and low falling-rising. Previous literature has shown that the acoustic realization of Thai lexical tones, namely their fundamental frequency or F0, is affected by the phonetic contexts in which they are produced. For example, in connected, conversational speech, Zsiga [(2006)] found that F0 contours of Thai tones produced in phrase-final position are different from those produced in non-phrase-final position. Context-dependent variation in F0 contours among lexical tones in Thai was also noted in Luksaneeyanavin [(1983)]. The main aim of this research was to further investigate F0 variations across the five lexical tones in Thai. Ten native Thai speakers ( 5 males and 5 female) were asked to produce Thai words in two speaking styles: slow, careful reading style; and fast, conversational style. Preliminary analyses revealed that: (1) the high-falling tone exhibited the highest degree of variation; (2) a greater amount of variation was observed among female speakers than among male speakers; and finally (3) a greater amount of variation was observed in open syllables than in closed syllables.

2aSC44. Quantifying the time-varying coordination between performer and audience. Adriano Barbosa (Linguist., Univ. of British Columbia, 2613 West Mall, Vancouver, BC, Canada, V6T 1Z4, adriano.vilela@gmail.com), Kevin G. Munhall (Queen's Univ., Kingston, ON, Canada, K7L 3N6), Hani C. Yehia (CEFALA, UFMG, Belo Horizonte, MG 31270-901, Brazil), and Eric Vatikiotis-Bateson (Univ. of British Columbia, 2613 West Mall, Vancouver, BC, Canada, V6T 1Z4)

This study examines the coordination that occurs ubiquitously during behavioral interaction (e.g., linguistic, social, musical). Coordination, however, does not imply strict synchronization. Musicians in a quartet, for example, will deviate slightly from the measured beat, alternately playing slightly ahead or behind. This paper showcases a new algorithm for computing the continuous, instantaneous correlation between two signals, at ANY temporal offset, resulting in a two-dimensional mapping of correlation and temporal offset that makes it possible to visualize and assess the timevarying nature of the coordination. The algorithm is demonstrated through analysis of the extraordinary performer-audience coordination evoked by Freddie Mercury during the rock group, Queen 's, Live Aid Concert at Wembley Stadium in 1985.

2aSC45. Perception of intonation as a function of sex. Yu-Wen Chen and Peter J. Watson (Dept. of Speech-Lang.-Hearing Sci., Univ. of Minnesota, 164 Pillsbury Dr. SE, Shevlin 115, Minneapolis, MN 55455)

Fitzsimons et al. [M. Fitzsimons, N. Sheahan, and H. Staunton, Brain Lang. 78, 94-108 (2001)] reported differences in the production of intonation between men and women during speech production. A relationship between the production and perception of segmental variables has been recognized [J. Perkell et al., Speech Commun. 22, 227-250 (1997)]; however, little information is available about the relationship of prosodic perception and production. Five men and five women listened to sentences produced by one man and one woman. The fundamental frequency (F0) of each sentence was resynthesized with 22 levels of F0 variation ranging from no variation to F0 variation that was 3.5 times greater than the typical variation of a sentence. For each sentence, a listener was randomly presented one of the levels of F0 variation and asked to adjust variation up and down until they were satisfied that the variation of that sentence sounded the most "natural." Additionally, each subject read a set of sentences aloud, and the F0 variation of these productions was analyzed. Results will be discussed in relation to the production and perception of intonation within and between the men's and women's groups.

2aSC46. Perceived sexual orientation and speech style: A perceptual and acoustic analysis of intentionally clear and conversational speech. Benjamin Munson (Dept. of Speech-Lang.-Hearing Sci., Univ. of Minnesota, 164 Pillsbury Dr., Minneapolis, MN 55455, munso005@umn.edu), Sarah Hargus Ferguson, and Catherine L. Connealy (Univ. of Kansas, Lawrence, KS 66045)

Munson et al. [Journal of Phonetics (2006)], found a correlation between perceived speech clarity and perceived sexual orientation (PSO) of male talkers: those who had been rated to sound gay were rated by an independent group to produce speech clearly. A smaller, opposite influence was found for women talkers. Munson et al. hypothesized that gay male speech styles might be based, in part, on clear speech styles. One weakness of Munson et al.'s study was that none of the speech examined was intentionally clear. This study follows up on this finding. A new group of 29 listeners rated the PSO of four men and four women producing conversational and intentionally clear speech, recorded as part of a larger study on interspeaker differences in talker intelligibility. PSO was rated using a five-point scale. Speech style had a moderate, statistically significant influence on PSO. In contrast with previous research, both men and women were rated as gayer-sounding when producing clear styles than when producing conversational ones. The differences in mean ratings were small. This investigation, thus, partly sup- 
ports Munson et al.'s findings. The relatively small influence of speech style on PSO likely reflects the complex, content-dependent nature of gay speech styles and PSO.

2aSC47. Sexuality and personality: The effect of pitch height and contour width on perception in Japanese. Margaret Camp (Dept. of East Asian Stud., Learning Services Bldg., Rm. 102, Univ. of Arizona, 1512 First St., P.O. Box 210105, Tucson, AZ, 85721-0105, mcamp@ arizona.edu)

This work investigates how Japanese listeners judge a speaker's personality characteristics and sexual orientation. Base sentences, produced in Japanese by a male and a female native Japanese speaker, were resynthesized to manipulate specific acoustic characteristics believed to influence perceived sexual orientation or personality. Overall, the higher the fundamental frequency (f0) of the female voice, the less likely it was to be perceived as lesbian. Similarly, the flatter the intonation pattern of the female voice, the more likely it was to be viewed as lesbian. For male voices, the higher the f0, the more likely the voice would be perceived as gay. Pitch variability did not play a significant role in this perception. Finally, out of the four personality scales investigated, two showed a significant correlation to lesbian voices: masculinity and calmness. Male voices showed the same pattern, with gay voices correlating with femininity and emotionality. The influence of the sex of the listener was also investigated, but male and female listeners appear to make similar judgments about most, but not all, characteristics.

2aSC48. An acoustic analysis of /æ/ variation and its relationship to perceived sexual orientation in American English. Benjamin Munson (Dept. of Speech-Lang.-Hearing Sci., Univ. of Minnesota, 164 Pillsbury Dr., Minneapolis, MN 55455, Munso005@umn.edu), Kathleen Currie Hall, and E. Allyn Smith (Ohio State Univ., Columbus, OH 43210)

Munson et al. [J. Phonetics (2006)] found that 11 self-identified gay and 11 heterosexual men produced different variants of the vowel /æ/, with gay men producing lower, more retracted variants and heterosexual men producing higher, more tense variants. Listeners' performance in a perception task in which they rated these talkers' sexual orientation was correlated with these measures: talkers with higher, more-tense /æ/ were rated as sounding more heterosexual than talkers with lower, more retracted /æ/. However, Smith et al. [New Ways of Analyzing Variation (2008)] found the opposite pattern in an experiment in which listeners rated the sexual orientation of productions by 10 trained talkers of sentences containing either tense or retracted /æ/ variants. A different group of listeners showed the same pattern when rating the /æ/ words excised from these sentences, though these listeners replicated Munson et al.'s original finding when presented with the $/ æ /$ words from the original 22 talkers. An attempt to reconcile these findings is made through detailed acoustic analysis of the /æ/ productions in the two experiments. Results underscore the importance of doing careful acoustic analyses in concert with careful phonetic transcription when conducting experiments on perception of social variables and speaker attributes.

2aSC49. Parametrization of $f 0$ characteristics of register ("Shiki") in mid-western Japanese dialects. Kenji Yoshida (Dept. of Linguist., Indiana Univ., 1021 E. 3rd St, Bloomington, 47405, keyoshid@umail.iu.edu)

Although the ToBI framework has achieved great success in modeling intonation in various languages, it may not capture the nature of certain aspects of f0 movements. This study models dynamic aspects of $f 0$ movement in "pitch register" contrasts observed in mid-western Japanese dialects, called "Shiki" by Japanese dialectologists. An exponential function [f0 = intercept $+b \times$ time $\times \exp (-c \times$ time $)]$ was fitted to the observed f0 movement of speakers of two Japanese dialects with Shiki contrasts (Kan'onji and Kishiwada). The results show that the two contrastive Shiki types are clearly discriminated in the 3-D parameter space [intercept $=$ baseline, $b=$ magnitude of f0-excursion, $c=$ inverse of peak timing]. Further analysis revealed that the two contrastive Shiki types are not reliably differentiated by the baseline. The more reliable predictors of Shiki types are the other two parameters that determine f0 movement within a word. The results are more congenial to a "dynamic" definition of Shiki system by Japanese dialectologists [e.g., Uwano, (1988)] and suggests that the analysis of Shiki as an initial f0 target [(High vs. Low, Pierrehumbert Beckman, 1988 for Osaka dialect] should be reconsidered.
2aSC50. Dialect distribution of North American English /r/. Suzanne Boyce (Dept. of Commun. Sci. and Disord., Univ. of Cincinnati, Cincinnati, OH 45267, boycese@email.uc.edu), Mark Tiede (Haskins Lab., New Haven, CT 06510), Kathy Groves-Wright (Cincinnati Veterans Affairs Medical Ctr., Cincinnati, OH 45220), Carol Espy-Wilson (Univ. of Maryland, College Park, MD 20742), Christy Holland, and Kyuran Choe (Univ. of Cincinnati, Cincinnati, OH, 45267)

It is well-known that North American English /r/ may be produced by a number of different tongue shapes. This was first reported in detail by Delattre and Freeman (1968), who examined x-ray data from 46 speakers from various regions of the United States and identified six basic tongue shapes used by speakers of "rhotic" dialects to produce /r/. It is unclear, however, whether different shapes are characteristic of particular dialects or geographic regions, or a matter of individual variation based on anatomy, developmental history, etc. In this study, we review findings from a number of published $\mathrm{x}$-ray and MRI studies of tongue shapes for $/ \mathrm{r} / \mathrm{using}$ speakers from various dialect regions. In addition, we characterize the variability of tongue shapes used for $/ \mathrm{r} /$ in an unpublished $\mathrm{x}$-ray database of elderly men with normal speech from the area of Cincinnati, OH. Results show that distinct tongue shape patterns in /r/ production occur both within and across dialect regions, suggesting the primacy of subject-specific factors over dialectal effects. [Work supported by NIH.]

2aSC51. Pitch accommodation in synchronous speech. Miran Kim (Dept. of Linguist., SUNY at Stony Brook, S201 SBS Bldg., Stony Brook, NY 11720, mrkim@ic.sunysb.edu) and Hosung Nam (Haskins Labs., New Haven, CT 06511)

Synchronously read speech, in which a pair of speakers reads a text together, has been shown to reduce variability in speech tempo and unveil underlying properties of speech competence. Our study question is whether a synchronous speech condition also influences a pitch domain as well as a temporal one, which has been the main focus in studies of synchrony. We investigated how tones are modulated in the synchronous speech condition. Eight speakers of Mandarin Chinese were instructed to read a story text alone and together in pairs. Speakers also read the text together with recorded speech. Each trial was repeated five times. We measured F0 at onset, center, and offset of a resonant period for each syllable and compared readalone to read-together speeches. Results show that (1) speakers in the synchronous speech condition tend to tune their pitch to each other such that F0 difference in pairs decreases overall; (2) they exhibit less variability in F0 across their own repetitions; (3) tonal targets in the read-together condition are achieved more stably in time. The pitch accommodation found in our study implies that speakers attend to F0 manifestation as well as tempo when F0 changes are phonologically contrastive in their language.

2aSC52. Effects of semantic context and regional dialect variation on speech intelligibility in noise. Cynthia G. Clopper (Dept. of Linguist., Ohio State Univ., 1712 Neil Ave., Columbus, OH 43210, clopper.1@ osu.edu)

Speech intelligibility in noise is affected by the semantic content of the utterance; semantically predictable utterances are more intelligible than semantically unpredictable or anomalous utterances. However, dialect variation interacts with semantic predictability in production; talkers produce more dialect-specific variants in semantically predictable contexts and more standard variants in semantically unpredictable contexts. The goal of the current study was to explore the interaction between dialect variation and semantic context in perception. Participants were asked to transcribe the final word of semantically predictable, unpredictable, and anomalous sentences produced by talkers from four regional dialects of American English and mixed with speech-shaped noise. The listeners exhibited an intelligibility benefit in the predictable context relative to the other two semantic contexts, regardless of talker dialect. However, differences in intelligibility between the dialects were reduced in the unpredictable and anomalous contexts relative to the predictable context, suggesting greater online adaptation to dialect variation in the unpredictable and anomalous contexts than the predictable context. These results are consistent with the finding that dia- 
lect differences may be attenuated in production in low predictability contexts, and suggest that perceptual dialect adaptation is affected by finegrained intratalker acoustic variation.

2aSC53. Investigating perceptual measures of speech alignment: Do matching tasks make the grade? Rachel M. Miller, Kauyumari Sanchez, and Lawrence D. Rosenblum (Dept. of Psych., Univ. of California, Riverside, 900 University Ave., Riverside, CA 92521)

Speech alignment is the tendency to produce speech which sounds similar to that of a person with whom one is speaking. It is often measured perceptually using an AXB-matching task [e.g., S. D. Goldinger, Psychol. Rev. 105, 251 (1998)]. In this procedure, naïve raters determine which of two utterances of a talker, produced prior (baseline) and after interaction with a model (e.g., shadowed), sounds more similar to that model. However, utilizing this type of perceptual measure has come under scrutiny due to its reliance on baseline utterances produced by talkers reading text. It has been suggested that AXB matching results reveal more about inherent differences between read and produced (shadowed) speech, than actual alignment. The present research addressed this concern. Experiment 1 involved a modified AXB task for which raters judged whether a subject sounded more like the model they had shadowed or another model. In Experiment 2, raters judged whether a subject sounded more similar to the model they had shadowed or to another subject who shadowed a different model. In both cases, results revealed that subjects sounded more similar to the model they shadowed.
This suggests that alignment is perceivable by raters even when removing the comparison to read text. [Work supported by NIDCD Grant R01DC008957.]

2aSC54. Identification of synthetic vowels based on time-varying vocal tract area functions. Kate Bunton and Brad H. Story (Dept. of Speech, Lang., \& Hearing Sci., Univ. of Arizona, P.O. Box 210071, Tucson, AZ 85721)

Identification accuracy for synthetic vowel stimuli generated with static vocal tract area functions from eight speakers was recently reported (K. Bunton and B. H. Story, JASA, in press). Although vowels were identified with reasonably high accuracy, in many cases neighboring vowels were confused. In the present study, new stimuli were generated based, again, on area functions from the same eight speakers. This time, however, the temporal variation of the vocal tract shape during natural production of isolated vowels was incorporated by developing a formant-to-area mapping for each speaker based on principal components analysis. This allowed formant contours extracted from recorded vowels to be mapped onto time-varying area functions. These were coupled to a voice source model and acoustic waves were propagated with a wave-reflection vocal tract model to generate vowels. Vowels were identified by listeners using a forced choice paradigm. Results indicated that including variation in vowel tract shapes, and hence formant frequencies, along with natural vowel durations improved identification accuracy relative to the previously tested static versions. It is concluded that these eight sets of area functions generate good renditions of target vowels when changes in vocal tract shape and duration were included. [Research supported by NIH R01-DC04789.]

TUESDAY MORNING, 19 MAY 2009

PARLOR B/C, 8:25 TO 11:40 A.M.

\title{
Session 2aSP
}

\section{Signal Processing in Acoustics and Underwater Acoustics: Detection and Classification of Underwater Targets I}

\author{
Patrick J. Loughlin, Cochair \\ Dept. of Bioengineering, Univ. of Pittsburgh, Pittsburgh, PA 15261 \\ Maya Gupta, Cochair \\ Dept. of Electrical Eng., Univ. of Washington, Seattle, WA 98195
}

Chair's Introduction-8:25

Invited Papers

$8: 30$

\begin{abstract}
2aSP1. Passive detection in deep water using the reliable acoustic path. Lisa M. Zurk (Elec. \& Comp. Eng. Dept., Portland State Univ., PO Box ECE 751, Portland, OR 97201, zurkl@pdx.edu)

The detection of quiet targets in deep water environments is of increasing interest for Navy applications, and detection performance depends critically on efficiently exploiting the frequency-dependent signal and noise structure. Previous measurements in the deep ocean environment of the northeast pacific has demonstrated the significant depth and frequency dependence of the noise, which can also vary with local wind conditions [Gaul et al., IEEE JOE (2007)]. The signal structure depends both on the spectrum of the target itself, but also on the propagation characteristics of the channel. In the concept of a reliable acoustic path (RAP), a receiver placed at or below the critical depth can detect shallow sources out to moderate ranges independent of near-surface water conditions or bottom interaction (hence, the identification as a "reliable" path). In this presentation, we present analysis of noise and signal structure for deep water channels from historic data, propagation modeling, and more recent experimentation. This structure is then used to compute signal excess to quantify detection performance as a function of receiver array placement and topology. Implications of the results on future Navy systems is discussed.
\end{abstract}


2aSP2. Enhancing passive automation performance using an acoustic propagation simulation. Ashley J. Llorens, Trudy L. Philip, Iman W. Schurman, and Cory R. Lorenz (Natl. Security Technol. Dept., JHU/APL, 11100 Johns Hopkins Rd., Laurel, MD 20723, ashley.llorens@jhuapl.edu)

During an at-sea encounter, signatures of interest can exhibit characteristics that differ from those observed in previously recorded data. These differences can occur due to variations in a number of factors including encounter geometry, propagation channel, and receiving sensor configuration. This paper presents a simulation technique that imposes low-frequency propagation effects on a timedomain signal using a normal mode method. High-quality, time-varying recorded signatures are used as inputs into the algorithm, which outputs band-limited time-series data for a selected geometry and environment. The output time-series are phased to simulate the timevarying pressure amplitudes that would be received by a towed array or any multielement passive sensor configuration operating in a realistic multipath environment. These capabilities enable the simulation of signatures of interest as captured under a broad range of littoral conditions by various passive sensors. These simulated data are used to augment scarce signature collections for training and assessing the performance of passive sonar automation.

\section{9:10}

2aSP3. Underwater target detection and classification using high resolution sensors and wide band sonar. Yvan Petillot, Yan Pailhas, Chris Capus, and Keith Brown (Oceans Systems Lab., Heriot-Watt Univ., UK)

In this presentation, our work on integrated target detection and identification using sonar systems is presented. Standard highresolution imaging sonar such as side scan and synthetic aperture are used for detection. Existing methods focus on statistical techniques using Markov models and machine learning-based techniques. Results on both synthetic and real data acquired in a large variety of terrains are presented. Identification can be based on either the shape of the target or its internal structure. Techniques-based active contours for shape analysis have been developed but these techniques only work well on very high-resolution data such as SAS and can be difficult to use on cluttered terrain. We propose to use the wide-band bio-sonar we developed to perform this task. In that case, the analysis focuses on the resonances induced by the internal structure of the target and the material used. Simulation confirmed by theoretical analysis on simple targets and experimental results demonstrate the effectiveness of this approach. These techniques are integrated on real vehicles in a collaborative vehicle framework to perform detection, classification, and identification of targets. [This work was supported by Thales, The Ministry of Defence, and the European Union under contract MRTN/CT/2006/036186.]

9:30

2aSP4. What do cognitive models and human judgments suggest about the desired structure of automatic classifiers? Jason E. Summers (SAIC, 10401 Fernwood Rd., Ste. 120, Bethesda, MD 20817-1133), Charles F. Gaumond, Colin W. Jemmott, and Derek Brock (Naval Res. Lab., Washington, DC 20375-5320)

Past studies probed human listeners' efficacy at classification of impulsive sonar echoes by using paired-comparison ratings to measure perceptual dissimilarity. Interpreting these ratings requires a cognitive model comprising both a representation of the stimuli and a process operating on that representation. Initially, perceived dissimilarities were represented as distances in Euclidean space via multidimensional scaling. This assumed a continuous and spatial cognitive representation and proved difficult to relate to a linear vector space of features. Later work by the authors suggested a discrete (categorical) cognitive representation may better reflect perception of these stimuli. While similarity-based classifiers can bypasses feature extraction [S. Philips and J. Pitton, J. Acoust. Soc. Am. 123, 3344 (2008) (A)], the form of the similarity measure reflects the assumed cognitive representation [L. Cazzanti and M. R. Gupta, Proc. IEEE Intl. Symposium Info. Theory, pp. 1836-1849 (2006)]. Here, findings based on paired-comparison ratings and verbal descriptions of stimuli are discussed in terms of the cognitive models they reflect and implications for classifier architecture: the form of similarity measures, whether features are continuous or discrete, and whether features are common to all classes. [Work supported by the Office of Naval Research.]

\section{Contributed Papers}

9:50

2aSP5. A generalized linear filtering approach for sonar receivers. Nabin S. Sharma and John R. Buck (Dept. of ECE, Univ. of Massachusetts Dartmouth, 285 Old Westport Rd., North Dartmouth, MA 02747, nsharma2 @umassd.edu)

Active sonar systems operating in realistic environments face the challenge of simultaneously detecting and resolving multiple targets. Receiver signal-to-noise ratio gain and main-lobe width of the receiver response to the transmitted signal limit the detection and resolution capability of an active sonar receiver, respectively. A matched filter is commonly used for target detection to exploit its optimal detection property. Theoretically, an inverse filter achieves optimal resolution and can be used to resolve targets. In practice, the inverse filter is difficult to implement and requires unrealistically high signal-to-noise ratio to perform well. For an active sonar receiver operating in a cluttered environment with closely spaced targets, a matched filter might not resolve some targets and an inverse filter could not detect some targets. For such environments, a generalized linear filtering approach is proposed. This new approach combines the matched and inverse filter properties in a controlled manner to achieve smooth trade off between detection and resolution. In simulation, the generalized linear filter output is compared with the matched and inverse filter outputs to demonstrate the advantages of the proposed filter. In addition, receiver operating characteristics of the filters confirm the improved performance of the generalized linear filter. [Work funded by ONR Code 321US.]

\section{0:05-10:25 Break}

\section{$10: 25$}

2aSP6. Pulse propagation and classification in time/frequency and position/wave number phase space. Patrick Loughlin (Depts. of Bioengineering and ECE, Univ. of Pittsburgh, 745 Benedum Hall, Pittsburgh, PA 15261, loughlin@pitt.edu)

In dispersive pulse propagation, different frequencies travel at different velocities, and, hence, the pulse is nonstationary in that it changes over time and distance. Joint phase space representations, such as the Wigner distribution and the spectrogram, are often employed to study nonstationary signals, and, hence, it is natural to consider their application to dispersive propagation. We consider position/wave number and time/frequency representations of a pulse propagating with dispersion and damping. We give the 
Wigner distribution of the pulse at a particular time/position in terms of the Wigner distribution of the initial pulse. Approximations of this result are derived, which are accurate and remarkably simple to apply, as compared to the stationary phase approximation. The approximations provide insights as well, in that they show how each point in phase space propagates at the group velocity, and lead to new features for classification, based on local phase space moments, that are invariant to the propagation effects of dispersion and damping. Examples relevant to sound propagation and classification in shallow water are presented. [Work supported by ONR Grant No. N00014-06-1-0009.]

\section{0:40}

2aSP7. Krylov methods in time-reversal imaging by multiple-signal-classification. Paul E. Barbone (Mech. Eng., Boston Univ., 110 Cummington St., Boston, MA 02215, barbone@bu.edu), Assad A. Oberai (Rensselaer Polytech. Inst., Troy, NY 12180), and Gonzalo R. Feijóo (Woods Hole Oceanograph. Inst., Woods Hole, MA 02543)

Multiple signal classification (MUSIC) has been used to form images and identify sound sources since 1986 [R. O. Schmidt, "Multiple emitter location and signal parameter estimation," IEEE Trans. Antennas Propag., AP34, 276-280 (1986)]. In active sonar imaging of point targets, the MUSIC method can be used to estimate the range of the time-reversal operator. In this context, the method is related to the decomposition of the time-reversal operator method. Typical implementations of these methods utilize measurements of the entire time-reversal operator, and require computations of its eigenvalues and eigenvectors. By contrast, we show that Krylov iterative methods can be used to perform MUSIC imaging with relatively few acoustic excitations. Furthermore, by using the Lanczos technique, no eigenvalues or eigenvectors need be computed. Rather, an orthonormal basis for the range space of the time-reversal operator can be constructed directly from the received data. Most of the necessary computing is performed by the array itself, performing as a kind of "analog computer." Finally, we show that useful images can be formed from one iteration to the next, while the data are being collected.

\section{0:55}

2aSP8. Automatic classification of underwater targets using fuzzy-cluster-based wavelet signatures. Hui Ou (Dept. of Elec. Engr., Univ. of Hawaii at Manoa, 2540 Dole St., Honolulu, HI 96822, ou@ hawaii.edu), John S. Allen, III, and Vassilis L. Syrmos (Univ. of Hawaii at Manoa, Honolulu, HI 96822)

A novel active acoustic underwater target recognition approach is applied to simulated mine-like targets. A feature extraction and integration method is introduced based on the continuous wavelet transform (CWT) and the Gustafson-Kessel (GK) clustering algorithm. CWT projects the acoustic signals onto the time-scale space, and the time-scale signatures are represented by a number of clusters. A modified GK clustering algorithm allows for specific enhancements: (i) autoadjustment of the shape of the clusters according to the time-scale patterns and (ii) autovalidation of the optimum number of clusters. The feature integration encompasses the application of the clustering analysis $N$ times. The resulting cluster centers are combined and overlaps are eliminated. Using this method, the target of interest is characterized by an integrated clustering distribution, or a feature template. A classification scheme is developed by matching the clustering distributions of the echoes received from an unknown target with the existing feature templates. White Gaussian noise has been added to the signals to simulate the interface of an ocean floor. The recognition rate varies from $72-98 \%$ for a SNR between $5-15 \mathrm{~dB}$.

\section{1:10}

2aSP9. Distributed detection and estimation based on the turbo approach. Michael Roan and Elizabeth Hoppe (Mech. Eng., Virginia Tech., 141 Durham Hall, Blacksburg VA 24060)

We develop a centralized information fusion architecture from basic principles of information theory and Bayesian statistics. It is well known that any clustering, quantizing, or thresholding of data causes loss of information unless a sufficient statistic is computed in the processing. For the case of wideband active ranging systems, the coherent output of an optimum beamformer and a matched filter is a sufficient statistic that can be transmitted to the fusion center. For unknown target velocity, range, and bearing, the wideband space-time matched filter output can be interpreted as a multidimensional wavelet transform or a delay-scale-bearing map. In this paper, an iterative, Bayesian, joint estimation-detection approach is used for computation of sufficient statistics and multisensor information fusion. An approach borrowed from sequential Bayesian processing is used to compute prior densities for joint Bayesian estimation-detection. In this approach $a$ posteriori densities become priors after a coordinate transformation that transforms the outputs of each sensor to a common reference frame for all sensors. In this paper, receiver operating characteristics and Cramer-Rao lower bounds are given for several undersea signal processing cases of interest.

\section{$11: 25$}

2aSP10. Variational Bayesian particle filtering for underwater target localization and tracking. T. Scott Brandes, Nilanjan Dasgupta, and Lawrence Carin (Signal Innovations Group, Inc., 1009 Slater Rd., Ste. 200, Res. Triangle Park, NC 27703)

Kalman filtering methods used in target tracking applications fail to perform satisfactorily when the true underlying posterior density of a target's location is non-Gaussian. These circumstances arise often in highly noisy environments dominated by strong scattering returns from clutter. Advanced tracking approaches, such as particle filters (PFs), that do not have the linear and Gaussian requirements of Kalman filtering are needed for target tracking in those complex environments. We used a variational Bayesian (VB) particle filter in our research to accurately track underwater targets simulated for such regimes. Particle filters rely on a Monte Carlo method of approximating the posterior distribution of an underwater moving target's location by a weighted set of sample points (particles) that evolve in time with the target. Variational Bayesian inference uses a Monte Carlo approach to estimate a distribution of the parameters of the posterior through a series of converging approximations. Our VB approach is shown to outperform the standard PF in both an active system (target tracking) and a passive system (source localization) in simulations. 


\title{
Session 2aUW
}

\section{Underwater Acoustics and Acoustical Oceanography: Mid- to High-Frequency Propagation and Scattering with Application to Underwater Communications}

\author{
Mohsen Badiey, Cochair \\ College of Marine and Earth Sciences, Univ. of Delaware, Newark, DE 19716-3501 \\ Daniel Rouseff, Cochair \\ Applied Physics Lab., Univ. of Washington, Seattle, WA 98105-6698
}

Chair's Introduction-8:00

Invited Papers

8:05

2aUW1. The dependence of the intensity of surface scattered signals on surface wave characteristics. James Preisig (Dept. of Appl. Ocean Phys. and Eng., Woods Hole Oceanograph. Inst., Woods Hole, MA 02543, jpreisig@ whoi.edu), Grant Deane (Univ. of California, La Jolla, CA 92093), and Chris Tindle (Univ. of Auckland, Auckland, New Zealand)

The compensation for the fluctuations in surface scattered signals remains one of the challenges for reliable and high rate underwater acoustic communications systems. Communication using phase coherent signals relies on the ability of the receiver to track the fluctuations in the impulse response of the channel between the transmitter and receiver. Techniques that can exploit a priori statistical characterizations of the fluctuations of the arrivals in the channel impulse response (fading statistics) will generally outperform systems that do not have access to such information. While fading statistics in radio communications is generally assumed to be Rayleigh or Rician, prior work [J. C. Preisig and G. B. Deane, J. Acoust. Soc. Am. 116, 2067-2080 (2004)] has shown that surface wave focusing can significantly change channel fading statistics. The physical basis for the intensity of surface scattered signals for both isolated specular scattering points and caustics developed in [C. T. Tindle and G. B. Deane, J. Acoust. Soc. Am. 117, 2783-2794 (2005)] relates intensity to ray geometry parameters. In this work, surface wave characteristics are related to both the source to receiver ranges at which normal focused specular scattering and caustics will occur and to the intensities of the surface scattered arrivals in each case. Experimental data are presented comparing theoretically predicted and measured intensities.

$8: 25$

2aUW2. Channel simulation for predicting acoustic modem performance. Michael B. Porter, Paul Hursky (HLS Res., 3366 N. Torrey Pines Ct., Ste. 310, La Jolla, CA 92037, mikeporter@hlsresearch.com), and Martin Siderius (Portland State Univ., Portland, OR 97207)

Acoustic modems usually provide the link in modern Distributed Netted Systems. However, their performance is strongly affected by the ocean weather as both the propagation medium and the source of background noise. Understanding these effects is important in pre-experiment planning, real-time adaptation, and retrospection (to ensure appropriate lessons are learned for future deployments). To this end, we have developed a channel simulator called VirTEX (Virtual Timeseries Experiment) that includes Doppler effects due to platform and ocean surface dynamics. VirTEX, in turn, is a component of the AComms Advisor, which provides links to ocean forecasts and other environmental information and uses HIL (Hardware in the Loop) testing to include a faithful modem simulation. This talk will discuss the process, as well as progress, in the algorithms.

\section{8:45}

2aUW3. Synthetic aperture communications exploiting fluctuations of the ocean environment. H. C. Song, W. S. Hodgkiss, W. A. Kuperman, T. Akal (Marine Physical Lab., Scripps Inst. of Oceanogr., UC San Diego, La Jolla, CA 92093-0238), and M. Stevenson (Spawar Systems Ctr., San Diego, CA 92152-5001)

Typically, fluctuations of the ocean environment pose difficulties for high-rate underwater acoustic communications. On the other hand, these fluctuations can be exploited in synthetic aperture communications (SAC) that take advantage of the relative motion between a transmitter and a receiver to create the equivalent of an array aperture. SAC requires that the channel impulse responses are sufficiently different from each other between transmissions for diversity combining to be effective. Rapid channel variations facilitate a shorter interval between transmissions resulting in a shorter integration time to achieve the desired diversity. This paper demonstrates high-rate SAC in shallow water exploiting fluctuations of the ocean environment. 


\section{Contributed Papers}

\section{9:05}

2aUW4. Coherent communications performance in environments with strong multi-pathing: Model/data comparison. Daniel Rouseff (Appl. Phys. Lab., College of Ocean and Fishery Sci., Univ. of Washington, Seattle, WA 98105)

Coherent underwater communications is hampered by the time spread caused by multi-pathing and the Doppler spread caused by the rapidly changing shallow water environment. In practice, a time-varying impulse response model will retain only a finite number of paths as useful signal and will be updated to compensate for the changing environment at a finite rate. In the present study, communications performance is examined as a function of the number of retained paths and the model-update interval. Results are quantified in terms of the mean squared error in the soft demodulation output. A physics-based performance model is developed. The model uses as input acoustic quantities like the Rayleigh parameter and environmental quantities like the significant wave height and the dominant period for surface waves. Model predictions for performance are compared to experimental results for data collected near the Hawaiian Island of Kauai. [Work supported by ONR.]

\section{9:20}

2aUW5. Ocean variability effects on high-frequency coherent acoustic communications. Aijun Song, Mohsen Badiey (Robinson Hall 114C, College of Marine and Earth Studies, Univ. of Delaware, Newark, DE 19716,), H. C. Song, and William Hodgkiss (Scripps Inst. of Oceanogr., La Jolla, CA 92093-0238)

The variability of the ocean environment can cause fluctuations of acoustic channels and these fluctuations often pose serious difficulties for high rate digital communications. During a series of recent acoustic communications experiments conducted around Kauai Island, Hawaii (KauaiEx'03, MakaiEx'05, and KAM'08), coherent communication data with concurrent environmental measurements were collected under different experimental settings. The variability of the ocean parameters, mainly the water temperature profiles and the sea surface condition, has been studied in order to understand their impact on the performance of acoustic communications at high frequencies (greater than $10 \mathrm{kHz}$ ). It is shown that both the long-term (in hours) changes in the ocean volume and the fast fluctuations (in seconds) of the sea surface have impact on the receiver design and on the receiver performance. Using time reversal combining followed by a single channel decision feedback equalizer, the ocean variability effects on the performance of coherent acoustic communications are shown as a function of receiver depth, source depth, and communication range.

\section{9:35}

2aUW6. Sonar simulation toolset modeling for underwater acoustic communications and networking. Julia B. Hsieh (Appl. Phys. Lab., Univ. of Washington, 1013 NE 40th St., Seattle, WA 98105-6698, julia@apl.washington.edu), Daniel Rouseff (Univ. of Washington, Seattle, WA 98105-6698), Aijun Song, and Mohsen Badiey (Univ. of Delaware, Newark, DE 19716)

The sonar simulation toolset (SST) is a ray-based propagation model capable of generating time series realizations with stochastic spreading of acoustic sequences. The model supports varying environments and geometries including moving sources and/or receivers. The simulated data were compared with experimental data collected at the Pacific Missile Range Facility, Kauai, Hawaii in 2003 (KauaiEx2003) and 2008 (KAM08). Models generated with appropriate sound speed profiles and wind speeds are shown to have similar channel impulse response functions to those observed during the experiments. The high-frequency communications sequences simulated from those models have been demodulated to demonstrate analogous results. These results suggest that underwater acoustic communications and networking can be effectively simulated with sonar simulation toolset to aid in experiment planning and data analysis. [Work supported by the Office of Naval Research.]

\section{9:50}

2aUW7. Basic research on time-reversal communication with a moving source-receiver. Takuya Shimura, Yoshitaka Watanabe, Hiroshi Ochi (Adv. Marine Tech. R\&D Program, JAMSTEC, 2-15 Natsushima-cho, Yokosuka 237-0061, Japan, shimurat@jamstec.go.jp), and Takehito Hattori (Nippon Marine Enterprises, Ltd., Yokosuka 238-0004, Japan)

A basic research on time reversal, to be used for communication with a long cruising AUV, has been researched by our research group. In such application, it is preferred to maintain its cruising speed. However, time reversal does not compensate the effect of moving source-receiver in principle. So in this study, simulations with a normal mode method considering the effect of source-receiver movement are executed in which the carrier frequency is $500 \mathrm{~Hz}$ and symbol rate is $100 \mathrm{bps}$. The method of combining time reversal and adaptive decision feedback equalizer is applied. In both cases of shallow water and the deep ocean, demodulation cannot be realized with only time reversal as expected, while the proposed method achieves communication at the moving speed up to $1.0 \mathrm{~m} / \mathrm{s}$.

\section{0:05-10:20 Break}

\section{0:20}

2aUW8. Impact of bottom type on orthogonal frequency division multiplexing in underwater communications. Nathan Parrish, Sumit Roy (Dept. of Elec. Eng., Univ. of Washington, Seattle, WA 98195-2500, nparrish@u.washington.edu), and Payman Arabshahi (Univ. of Washington, Seattle, WA 98105-6698)

The impact of bottom sediment type in relation to acoustic communications via orthogonal frequency division multiplexing (OFDM) is shown via experimental results and simulation. Experimental data from Lake Washington, Seattle with a "silty clay" bottom show that the multipath delay spread is longer at $250 \mathrm{~m}$ than at $4 \mathrm{~km}$. This results in better OFDM performance at the longer range. Similar results are shown via simulation using a channel model developed from Bellhop, a Gaussian Ray tracing tool [M. Porter, "Bellhop Gaussian beam/finite element beam code," Available: http://oalib .hlsresearch.com/Rays/index.html (2007)]. Through simulation, results are also shown under similar conditions to the experiment but with varying bottom type. The results show that the performance of OFDM signaling is dependent on the bottom type as well as specific source/receiver geometry. [Work supported by NASA ESTO.]

\section{$10: 35$}

2aUW9. Capacity of orthogonal frequency division multiplexing systems over fading underwater acoustic channels. Chantri Polprasert, James A. Ritcey (Dept. of Elect. Eng., Univ. of Washington, Seattle, WA 98195-2500, ritcey@ee.washington.edu), and Milica Stojanovic (Northeastern Univ., Boston, MA 02115)

The channel capacity bounds of orthogonal frequency division multiplexing (OFDM) systems over the underwater (UW) acoustic fading channel as a function of the distance between the transmitter and receiver are investigated. The upper bound is obtained under perfect channel state information (CSI) assumption at the receiver. The lower bound is obtained under imperfect CSI at the receiver. The reduction from the upper bound is due to the linear minimum mean square prediction error. The UW channel deviates from the wide sense stationary and uncorrelated scattering (WSSUS) model commonly used for small bandwidths. Frequency-selectivity is incorporated due to the acoustic propagation into each arrival path between the transmitter and receiver. This leads the UW channel to be modeled as a frequencydependent doubly spread fading channel characterized by the wide sense stationary and correlated scattering (WSS-non-US) fading assumption. Both Rayleigh and Ricean fading assumptions are investigated in the model. Results from the model show a gap between the upper and lower bounds that depends not only on the ranges and shape of the scattering function of the UW channel but also the distance between the transmitter and receiver. The model for the scattering function was confirmed from experimental data. 
10:50

2aUW10. Multichannel combination investigations for differential frequency hopping transmissions in shallow water. Dianne E. Egnor, Geoffrey S. Edelson (BAE Systems, Advvaned Systems \& Technol., P.O. Box 868, Nashua, NH 03061-0868), Luca Cazzanti, and Julia Hsieh (Univ. of Washington, Seattle, WA 98105)

Underwater acoustic communication requires waveforms that are robust to the signal distortions caused by the acoustic channel. Many waveforms used for this purpose require the transmission of training symbols that span the intersymbol interference to "learn" and compensate for these channel effects. These waveforms also require exacting coordination between the transmitters to avoid multiple access interference. Differential frequency hopping (DFH) is a fast frequency hopping, digital signaling technology that requires minimal information at the transmitter to communicate in the underwater channel. DFH has the desirable performance features of noninterfering spread spectrum operation, spectral reuse, and fading and interference resistance. This presentation describes the baseline autosynchronizing, single-user DFH decoder for a multiple hydrophone receiver and investigates two processing techniques incorporated for shallow-water multiuser applications: fading mitigation and multiuser interference mitigation, as implemented in conjunction with array processing. We present the performance of the baseline DFH decoder in terms of bit error rate with and without these enhancements on single- and multiuser data collected at sea during the 2008 Surface Processes and Acoustic Communications Experiment (SPACE08).

\section{1:05}

2aUW11. Modeling differential frequency hopping communication in the underwater acoustic channel. Luca Cazzanti, Julia Hsieh (Appl. Phys. Lab., Univ. of Washington, Box 355640, Seattle, WA 98105), Dianne Egnor, and Geoffry S. Edelson (BAE Systems, Nashua, NH 03061)

Differential frequency hopping (DFH) is a fast frequency hopping digital modulation scheme with proven multiple-access and jamming robustness properties in typical wireless channels. Characterizing the capabilities of DFH modulation in the more challenging underwater acoustic channel requires careful analyses that rely on both computer simulations and data collected at sea. The Sonar Simulation Toolkit (SST) is used to model challenging underwater environments and simulate the propagation of DFH waveforms in the corresponding underwater acoustic channels. Our simulations provide baseline performance results that can be used to assess the capabilities of DFH modulation and guide future algorithmic improvements to the receiver. In particular, our simulations show that incorporating equalization techniques into the DFH baseline receiver leads to improved decoding performance in challenging environments characterized by long channel impulse responses, which are known to cause inter-symbol interference in the received acoustic bit stream. To ensure their relevance to practical applications, our computer-based SST models are calibrated to the environmental parameters measured during recent at-sea experiments, including the Rescheduled Acoustic Communications Experiment (RACE08), and the corresponding performance is analyzed.

\section{1:20}

2aUW12. Achieving maximum space-time-frequency diversity in shallow water acoustic channels: System design and experimental results. Costas Pelekanakis and Arthur B. Baggeroer (5-206, Ctr. for Ocean Eng., Dept. of Mech. Eng., Massachusetts Inst. of Technol., 77 Massachusetts Ave., Cambridge, MA 02139, gas@mit.edu)

This work sheds light on the individual impact of coded modulation and diversity in achieving reliable underwater acoustic (UWA) communications. Two systems with the same bit-rate and complexity but different diversity order are designed and compared. The "conventional" system combines trellis coded modulation (TCM), symbol interleaving and an 8PSK signal set. The "alternative" or bit-interleaved coded modulation (BICM) system combines a convolutional code, bit interleaving, and a 16QAM signal set. Both systems are coupled with orthogonal frequency division multiplexing (OFDM) to cope with the intersymbol interference (ISI). When two or three projectors are used, the above coding schemes are concatenated with spacetime block codes (STBC). Bit-error rates using data recorded during the RACE'08 off the coast of Narragansett, USA are computed demonstrating the superiority of BICM schemes relative to their TCM counterparts over an extensive collection of shallow water channels, thus proving that BICM is a promising technique towards achieving reliable UWA communications. In particular, by using BICM and $4 \mathrm{kHz}$ of bandwidth, we have successfully accomplished a $7150 \mathrm{~b} / \mathrm{s}$ transmission rate over $1 \mathrm{~km}$ range.

\section{1:35}

2aUW13. Improving underwater vehicle communication in the littoral zone through adaptive vehicle motion. Toby E. Schneider and Henrik Schmidt (Ctr. for Ocean Eng., Dept. of Mech. Eng., MIT, 77 Massachusetts Ave., Cambridge, MA 02139, tes@mit.edu)

Autonomous underwater vehicles (AUVs) are increasingly being used in clusters to perform detecting and sensing tasks in the littoral zone. These tasks require substantial data throughput between underwater and surface nodes. Acoustic modems provide the only practical means of sending these data, but sound in the littoral waveguide is heavily refracted downward and multiply reflected by the shallow bottom. These effects lead to shadow zones where messages are lost and convergence zones that allow for longer range receipt of high bitrate messages. During the GLINT08 sea trials, messages were sent using a multiple rate $25-\mathrm{kHz}$ acoustic modem (the WHOI "MicroModem") between several undersea and surface nodes. The message quality (mean square error) was analyzed and compared to dynamic ray tracing models of the transmission loss, using environmental data measured by the AUVs and the research vessel. Significant correlation was found between the data and the modeled acoustic field, suggesting that such models could be run on-board the AUVs to predict regions of low transmission loss. This would give the AUVs predictive ability to choose when to send messages to maximize the probability of receipt and the choice adaptively move to improve chances of successful message reception. 


\section{Meeting of Standards Committee Plenary Group}

to be held jointly with the meetings of the

ANSI-Accredited U.S. Technical Advisory Groups (TAGs) for:

ISO/TC 43, Acoustics,

ISO/TC 43/SC 1, Noise,

ISO/TC 108, Mechanical vibration, shock and condition monitoring,

ISO/TC 108/SC 2, Measurement and evaluation of mechanical vibration and shock as applied

to machines, vehicles and structures,

ISO/TC 108/SC 3, Use and calibration of vibration and shock measuring instruments,

ISO/TC 108/SC 4, Human exposure to mechanical vibration and shock,

ISO/TC 108/SC 5, Condition monitoring and diagnostics of machines,

ISO/TC 108/SC 6, Vibration and shock generating systems,

and

IEC/TC 29, Electroacoustics

P. D. Schomer, Chair

U.S. Technical Advisory Group (TAG) for ISO/TC 43 Acoustics and ISO/TC 43/SC 1 Noise

2117 Robert Dr., Champaign. IL 61821

D. J. Evans, Chair

U.S. Technical Advisory Group (TAG) for ISO/TC 108 Mechanical vibration shock and condition monitoring, and ISO/TC 108/SC 3 Use and calibration of vibration and shock measuring devices

National Inst. of Standards and Technology, 100 Bureau Dr., Stop 8220, Gaithersburg, MD 20899

W. C. Foiles, Co-Chair

U.S. Technical Advisory Group (TAG) for ISO/TC 108/SC 2 Measurement and evaluation of mechanical vibration and shock as applied to machines, vehicles and structures

BP America, 501 Westlake Park Blvd., Houston, TX 77079

R. Taddeo, Co-Chair

U.S. Technical Advisory Group (TAG) for ISO/TC 108/SC 2 Measurement and evaluation of mechanical vibration and shock as applied to machines, vehicles and structures

NAVSEA, 1333 Isaac Hull Avenue, SE, Washington Navy Yard, Washington, DC 20376

D. D. Reynolds, Chair

U.S. Technical Advisory Group (TAG) for ISO/TC 108/SC 4 Human exposure to mechanical vibration and shock 3939 Briar Crest Ct., Las Vegas, NV 89120

D. J. Vendittis, Chair

U.S. Technical Advisory Group (TAG) for ISO/TC 108/SC 5 Condition monitoring and diagnostics of machines 701 Northeast Harbour Ter., Boca Raton, FL 33431

R. Taddeo, Vice Chair

U.S. Technical Advisory Group (TAG) for ISO/TC 108/SC 5 Condition monitoring and diagnostics of machines NAVSEA, 1333 Isaac Hull Avenue, SE, Washington Navy Yard, Washington, DC 20376

\section{Peterson, Chair}

U.S. Technical Advisory Group (TAG) for ISO/TC 108/SC 6 Vibration and shock generating systems

200 Dixie Ave., Kalamazoo, MI 49001

V. Nedzelnitsky

U.S. Technical Advisor (TA) for IEC/TC 29 Electroacoustics

National Inst. of Standards and Technology, 100 Bureau Dr., Gaithersburg, MD 20899-8221 
The reports of the Chairs of these TAGs will not be presented at any other $\mathrm{S}$ Committee meetings.

The meeting of the Standards Committee Plenary Group will precede the meetings of the Accredited Standards Committees S1, S3, S3/SC 1, and S12 which are scheduled to take place in the following sequence:

ASC S3, Bioacoustics

ASC S3/SC 1, Animal Bioacoustics

ASC S1, Acoustics

ASC S12, Noise

\author{
Tuesday, May 19 \\ Tuesday, May 19 \\ Tuesday, May 19 \\ Tuesday, May 19
}

$$
\begin{aligned}
& \text { 10:30 a.m. - 11:30 a.m. } \\
& \text { 11:45 a.m. - 1:00 p.m. } \\
& \text { 2:45 p.m. - 3:45 p.m. } \\
& \text { 4:00 p.m. - 5:00 p.m. }
\end{aligned}
$$

Please note that ASC S2, Mechanical Vibration and Shock will meet in advance of the Plenary meeting, on Monday, May 18 at 4:30 p.m.

Discussion at the Standards Committee Plenary Group meeting will consist of national items relevant to all S Committees and U.S. TAGs.

The U.S. Technical Advisory Group (TAG) Chairs for the various international Technical Committees and Subcommittees under ISO and IEC, which are parallel to S1, S2, S3, and S12 are as follows:

\section{U.S. TAG Chair/Vice Chair}

$\underline{\text { TC or SC }}$

\section{ISO}

P. D. Schomer, Chair

P. D. Schomer, Chair

D. J. Evans, Chair

W. C. Foiles, Co-Chair

R. Taddeo, Co-Chair

D. J. Evans, Chair

D. D. Reynolds, Chair

D. J. Vendittis, Chair

R. Taddeo, Vice Chair

C. Peterson, Chair

IEC

V. Nedzelnitsky, U.S. TA tures
ISO/TC 43 Acoustics

ISO/TC 43/SC1 Noise

ISO/TC 108 Mechanical vibration, shock and condition monitoring

ISO/TC 108/SC2 Measurement and evaluation of mechanical vibration and shock as applied to machines, vehicles and struc-

ISO/TC 108/SC3 Use and calibration of vibration and shock measuring instruments ISO/TC 108/SC4 Human exposure to mechanical vibration and shock ISO/TC 108/SC5 Condition monitoring and diagnostics of machines

ISO/TC 108/SC6 Vibration and shock generating systems

$\underline{\text { U.S. Parallel Committee }}$
$\mathrm{S} 1 / \mathrm{S} 3$

$\mathrm{S} 12$

S2

$\mathrm{S} 2$

$\mathrm{S} 2$

$\mathrm{S} 2 / \mathrm{S} 3$

$\mathrm{S} 2$

$\mathrm{S} 2$

IEC/TC 29 Electroacoustics 


\title{
Meeting of Accredited Standards Committee (ASC) S3 Bioacoustics
}

\author{
C. A. Champlin, Chair S3 \\ Univ. of Texas, Dept. of Communication Sciences \& Disorders, CMA 2-200, Austin, TX 78712 \\ D. A. Preves, Vice Chair S3 \\ Starkey Laboratories, Inc., 6600 Washington Ave., S. Eden Prairie, MN 55344
}

\begin{abstract}
Accredited Standards Committee S3 on Bioacoustics. Working group chairs will report on the status of standards under development. Consideration will be given to new standards that might be needed over the next few years. Open discussion of committee reports is encouraged.
\end{abstract}

People interested in attending the meeting of the TAGs for ISO/TC 43 Acoustics and IEC/TC 29 Electroacoustics, take note - those meetings will be held in conjunction with the Standards Plenary meeting at 9:00 a.m. on Tuesday, 19 May 2009.

Scope of S3: Standards, specifications, methods of measurement and test, and terminology in the fields of psychological and physiological acoustics, including aspects of general acoustics which pertain to biological safety, tolerance and comfort.

TUESDAY MORNING, 19 MAY 2009

ALEXANDERS LOUNGE, 11:45 A.M. TO 1:00 P.M.

\section{Meeting of Accredited Standards Committee (ASC) S3/SC 1 Animal Bioacoustics}

\author{
D. K. Delaney, Chair S3/SC 1 \\ USA CERL, 2092 Farber Dr., Champaign, IL 61822 \\ M. C. Hastings, Vice Chair S3 \\ 6422 Crosswoods Dr., Falls Church, VA 2204412144
}

\begin{abstract}
Accredited Standards Committee S3/SC 1 on Animal Bioacoustics. Working group chairs will report on the status of standards under development. Consideration will be given to new standards that might be needed over the next few years. Open discussion of committee reports is encouraged.

Scope of S3/SC 1: Standards, specifications, methods of measurement and test, instrumentation and terminology in the field of psychological and physiological acoustics, including aspects of general acoustics which pertain to biological safety, tolerance and comfort of non-human animals, including both risk to individual animals and to the long-term viability of populations. Animals to be covered may potentially include commercially-grown food animals; animals harvested for food in the wild; pets; laboratory animals; exotic species in zoos, oceanaria or aquariums; or free-ranging wild animals.
\end{abstract}




\title{
Session 2pAA
}

\author{
Architectural Acoustics: Computer Auralization \\ Robert C. Coffeen, Chair
School of Architecture and Urban Design, Univ. of Kansas, Lawrence, KS 66045 \\ Chair's Introduction-1:00
}

Invited Papers

1:05

2pAA1. Auralization-audibility of changing scattering coefficients for audience areas in different size venues. Sarah Rollins (Sparling, Inc., 720 Olive Way, Ste. 1400, Seattle, WA 98101, srollins@sparling.com)

Auralizations in computer modeling are affected by many different variables, such as absorption coefficients, scattering coefficients, and the complexity of the model. However, scattering coefficients are often determined by the best guess of the consultant. In order to isolate the changes due to the scattering coefficient for comparison with measured data, a simple rectilinear room was measured and modeled. This paper will show how changing the architectural surface and audience scattering coefficients affects the auralizations for this room. For comparison, the effects of changing these same coefficients in a larger venue will also be presented.

\section{$1: 25$}

2pAA2. Comparison of modeled versus measured room acoustic parameters in the recently completed Shaghoian Concert Hall in Clovis, California. Bill Dohn (Dohn and Assoc., Inc., 630 Quintana Rd. 312, Morro Bay, CA 93442, bill.dohn@ gte.net) and Richard H. Campbell (Bang-Campbell Assoc., Falmouth, MA 02536)

The Shaghoian Concert Hall in Clovis, CA is a popular new variable acoustic music venue in the greater Fresno, CA area. The design process for this hall included CATT-Acoustic (TM) modeling for analysis and verification of essential room acoustic parameters based upon the settings of the variable acoustic devices. The model of the final design produced parameters that were closely in line with ideal values for a hall of this size and use. Model results will be compared to measured parameters in the completed hall.

$1: 45$

2pAA3. From toy to tool-advanced auralization procedure in daily design work. Wolfgang Ahnert and Stefan Feistel (AFMG, Arkonastr. 45-49, 13189 Berlin, Germany)

Since more than 20 years simulation procedures are more and more common, but the auralization procedure is considered mainly as a toy. The reason for this relates partially to the efforts needed to get real auralized sound files. In a previous paper the authors reported about a method to produce high end auralization files. This method allows to use normal head phones to get the right spatial impression without in-head localization. The main reason not to use an auralized sound impression in the daily design work is the time-consuming procedure to calculate true impulse responses (IR) especially in large halls. In this paper new algorithms are reported to calculate IRs in multithread mode with one computer or better via a network with a set of CPUs working in parallel. So calculations in large halls maybe be reduced from 8-10 h with one CPU to 10-20 min with multicore computers. The paper explains the way from the model to high-end auralized files. Some examples demonstrate the quality of these derived binaural files.

\section{2:05}

2pAA4. The spatial perception of a large pipe organ in various locations in a church computer model is investigated using multiple sources properly spaced within an organ chamber, with each source representing an organ stop. Richard Campbell (Bang-Campbell Assoc., 26 G Chilmartk Dr. East Falmouth, MA 02536)

It is surmized that the auralization of a large pipe organ should use multiple sources properly spaced in the organ chamber with each source representing a stop. But at some point in the acoustic environment of the listener, the advantage of such spatial sourcing disappears. A MIDI sequencer is used to generate audio files of a Buxtahuda fugue in six parts using six different stops. The sources are properly spaced within an organ chamber in a large church. Binaural recordings are evaluated at various locations in the church.

$$
\text { 2:25 }
$$

2pAA5. Comparison of auralized and measured sound in moderately reverberant spaces. Robert C. Coffeen, Jonathan Birney, Stephanie Hoeman, Shane Kanter, Hannah Schultheis (School of Architecture and Urban Planning, Univ. of Kansas, 1465 Jayhawk Blvd., Lawrence, KS 66045, coffeen@ku.edu), Lauren M. Ronsse, and Lily M. Wang (Univ. of Nebraska-Lincoln, Omaha, NE 681820681) 
This research performs an in-depth comparison of sound from computer auralizations with measured binaural room impulse responses and recordings taken in actual spaces. Binaural measurements and recordings have been gathered in two church naves with midfrequency reverberation times of about 1.5 and $2.5 \mathrm{~s}$. The results from these measurements are compared with auralizations from computer models of the spaces in two different room acoustic modeling programs. The source and receiver positions used for the auralizations are the same as those measured in the real spaces. One of the naves was previously investigated [R.C. Coffeen and G. Caunt, J. Acoust. Soc. Am. 105, 1174 (1999) (A)] with results from computer auralizations compared to measured binaural recordings in the space. As numerous advancements have been made to computer modeling programs since that time, this project revisits and expands the previous research using updated modeling and measurement techniques. The results of this research not only portray the developments in computer modeling that have occurred over the past decade, but also depict the relationship between current computer auralizations and actual sound in moderately reverberant spaces.

\section{2:55}

2pAA6. Using computer auralization to help prevent major problems relating to room acoustics and electroacoustic sound reinforcement. Robert C. Coffeen (Sch. of Archit. and Urban Plan., Univ. of Kansas, 1465 Jayhawk Blvd., Lawrence, KS 66045, coffeen@ku.edu)

Computer modeling and computer auralization will produce comparative listening experiences for architects, building owners, and building users based on selected interior surface shapes, interior finish materials, and electroacoustic sound distribution. Auralization can be a significant tool in helping building designers, owners, and users understand potential acoustical problems and the need for proper acoustic and electroacoustic design.

\section{Contributed Papers}

\section{3:15}

2pAA7. Diffraction culling for virtual-acoustic simulations. Paul Calamia (Dept. of Comp. Sci., Princeton Univ., 35 Olden St., Princeton, NJ 08540 , pcalamia@cs.princeton.edu), Benjamin Markham (Acentech Inc., Cambridge, MA 02138), and U. Peter Svensson (Norwegian Univ. of Sci. and Technol., NO-7491 Trondheim, Norway)

Acoustic simulations of complex virtual environments typically are created with geometrical-acoustics techniques. Such simulations can be augmented with edge diffraction modeling for improved accuracy, but not without a significant increase in processing time due to the additional propagation paths which must be considered and the computational complexity of the diffraction calculations. However, for a given modeling scenario, the contribution of a diffracted path to the overall impulse response can vary over a large range, suggesting that certain diffracted paths can be ignored, or culled, to reduce processing time with a limited effect on the accuracy of the simulation. In this talk, we first analyze the effects of diffraction culling through a precomputed, amplitude-based ranking scheme. We then describe a simple procedure for identifying and culling insignificant diffraction components during a virtual-acoustic simulation which approximates the performance of the precomputed ranking. Through numerical and subjective analysis, we show that a significant percentage of diffracted paths can be ignored if the retained paths are those which lead to the highestamplitude diffraction components, although the audible effects of such diffraction culling are dependent on the input signal.

\section{3:30}

2pAA8. Singers' preferences for acoustical characteristics of performing spaces. Kathleen Stetson (Arup Acoust., 155 Ave. of the Americas, New York, NY 10013, kathleen.stetson@arup.com) and Jonas Braasch (Rensselaer Polytechnic Inst., Troy, NY 12180)

Classical singers, whose instruments' close proximity to their ears makes them unique among musicians, require particular attention from acousticians addressing musician support in performance space design. This study expands upon the few previous analyses of acoustics for singers by exploring what is most basic about solo voice self-perception in halls used for concerts and recitals. A questionnaire was given to a number of professional classical singers and their numerical and narrative responses were analyzed. Five concert halls were measured from the singer's perspective on stage, utilizing a head and torso simulator with a mouth speaker to mimic the proximity of a singer's voice and ears. The resulting impulse responses were utilized in preference tests featuring real-time binaural auralization of singers' vocalizations. The correlations between the subjective results, existing objective parameters, and physical hall characteristics were explored. A strong connection was found between increasing preference and increasing reverberation time. Additionally, test subjects indicated a statistically significant dislike of or preference for a hall, regardless of the singing location on stage or the classical genre being sung. Further developments of this study are discussed in the context of ongoing opera house design utilizing computer model simulations.

\section{$3: 45$}

2pAA9. Thirteen findings in ten minutes. Michael Ermann, Nate Crawford, Braden Field, Jessica Green, Sky Kim, Julia Mitchell, Steve Smith, Matthew Van Wagner, John Samuel Victor, and Amanda Massengill (School of Architecture and Design, Virginia Tech, 201 Cowgill Hall, Blacksburg, VA 24061-0205, mermann@vt.edu)

Nine architecture students in an architectural acoustics course studied the influence of design on the acoustic response of spaces. Thirteen of their projects will be presented, rapidly, with time to delve into some of them more deeply in response to questions from attendees. Areas of focus include: room shaping and balcony composition; ceiling and canopy height; canopy design; perception and preference of double sloped decays; side wall angle and orientation; room length; very tall ceilings; room width; side wall diffusion; mapping background noise levels; and frequency content of noise. 


\title{
Session 2pAB
}

\section{Animal Bioacoustics and Acoustical Oceanography: Autonomous Remote Monitoring Systems for Marine Animals II}

\author{
Kathleen C. Stafford, Chair \\ Applied Physics Lab., Univ. of Washington, Seattle, WA 98105
}

Invited Papers

1:00

2pAB1. Validating acoustic monitors for marine animals: Field experience with beaked whales and digital acoustic monitors (DMONs). Mark Johnson, Tom Hurst (Woods Hole Oceanograph. Inst., 86 Water St., Woods Hole, MA 02543, majohnson@whoi.edu), Anton Arias, and Natacha Aguilar de Soto (Univ. of La Laguna, Tenerife, Spain)

Passive acoustic monitoring (PAM) is a promising tool for improving the effectiveness of visual surveys for marine animals and for stand-alone persistent monitoring. Nonetheless, significant data gaps make it difficult to predict PAM performance for many species. Detection functions are only beginning to be formed for some vocalizations and detectors. Statistical models for calling rates, as a function of behavior, are needed to translate acoustic detections into abundance estimates, or lack of detections into decisions about absence of animals. Finally, ambient noise levels and spectra are unavailable in most of the world's oceans, complicating the application of results from one area to another. Although some of these gaps are being addressed by a growing group of researchers, there is an urgent need to develop standards for measuring and reporting results, and to foster the interchange of data. To exemplify the form such standards might take, and the potential pitfalls, we present results from a series of field experiments designed to assess the performance of an acoustic monitor for beaked whales. The experiments made use of a freely available reference design for an acoustic detector, called the DMON, which may provide a basis for standardizing and validating real-time implementations.

\section{$1: 20$}

2pAB2. Screening large data sets and real-time data streams for bioacoustic signals. Holger Klinck (Cooperative Inst. for Marine Resour. Stud., and NOAA Pacific Marine Environ. Lab., Oregon State Univ., 2030 SE Marine Sci. Dr., Newport, OR 97365, Holger.Klinck@oregonstate.edu), Lars Kindermann (Alfred Wegener Inst. for Polar and Marine Res., 27568 Bremerhaven, Germany), David K. Mellinger (Oregon State Univ., Newport, OR 97365), and Olaf Boebel (Alfred Wegener Inst. for Polar and Marine Res., 27568 Bremerhaven, Germany)

This presentation will discuss a major challenge of passive-acoustic monitoring systems: the analysis of large data sets to identify the occurrence of bioacoustic signals of interest. Triggered by the rapid development of digital audio technology and the increasing capacity of memory devices, it has become easier than ever to produce large, long-term acoustic data sets. However, analyzing these data sets is challenging, as suitable automated detection and classification systems are needed to perform the analysis in a reasonable amount of time. One such large data set is currently produced by the Perennial Acoustic Observatory in the Antarctic Ocean. This passive acoustic observatory has been operated by the Alfred Wegener Institute since January 2006 and has generated more than 10,000 hours of data to date. The observatory features a data link via satellite, which allows analysis of the recorded hydroacoustic data in real time in Germany. However, to be able to run custom algorithms over the entire data set in several times real-time speed, a distributed computing system was developed and applied. Here we provide a detailed description of this system and discuss further possible applications.

\section{$1: 40$}

2pAB3. Acoustic sampling for marine mammals in the Beaufort Sea July 2007-March 2008. Kathleen Stafford (Appl. Phys. Lab, Univ. of Washington, 1013 NE 40th St., Seattle WA 98105, stafford@apl.washington.edu), Sue Moore (Alaska Fisheries Sci. Ctr., Seattle WA 98115), Catherine Berchok (Natl. Marine Mammal Lab, Seattle WA 98115), and David K. Mellinger (Oregon State Univ., Newport OR 97365)

As climate change is driving rapid, unprecedented warming of the Arctic, there is increasing interest in how such change will impact Arctic marine mammals. Impacts are anticipated from from habitat alteration, including increasing ambient noise levels from shipping, seismic exploration for oil and gas and geophysical research, and (potentially) commercial fishing. In order to monitor natural and anthropogenic sources of noise, four autonomous recorders were deployed along the 100-m isobath between Cape Halkett and Barrow and recorded data from July 2007-March 2008. The instruments sampled at $8192 \mathrm{~Hz}$ on a schedule of $10 \mathrm{~min}$ on, $20 \mathrm{~min}$ off. Marine mammal sounds recorded included pinnipeds (walrus and bearded seals) and cetaceans (bowhead and beluga whales), while anthropogenic sources included shipping and air gun sounds. Seasonal and geographic patterns for these sounds will be presented. These data form part of a broader-scale international, year-round monitoring program in the Arctic that we hope will eventually span the entire Arctic and provide a basin-wide acoustic observatory. 


\section{Contributed Papers}

\section{2:00}

2pAB4. Integration of automated detection methods into NOAA Southwest Fisheries Science Center (SWFSC) acoustic marine mammal monitoring protocol. Tina M. Yack, Jay Barlow, Shannon Rankin (Natl. Marine Fisheries Svce., Southwest Fisheries Sci. Ctr., 3333 N. Torrey Pines Court, La Jolla, CA 92037, tina.yack@noaa.gov), and Douglas Gillespie (Univ. of St. Andrew, Fife KY16 8LB, Scotland)

Southwest Fisheries Science Center (SWFSC) has used combined visual and acoustic techniques to monitor marine mammal populations for the past 8 yrs. Currently, SWFSC passive acoustic surveys of cetaceans require specially trained personnel to monitor hydrophone signals in real-time. While effective, this method is time-consuming and costly. Automated detection of cetacean vocalizations would be a valuable tool during SWFSC surveys, allowing for detection when experienced technicians are unavailable. This technique is advantageous because it significantly reduces effort and removes sources of human error and bias in detection ability. PAMGUARD 1.0 CORE software was evaluated for use in automated detection of cetacean acoustic signals. Three different detector configurations of PAMGUARD were evaluated. This work shows that the majority of whistle and click events can be detected using PAMGUARD software. All of the PAMGUARD trials were capable of detecting whistles and clicks of cetacean species with varying success. These techniques were field-tested at sea during a recent SWFSC marine mammal survey. Automated detection of beaked whales and Dall's porpoise during this survey will also be discussed. It is our goal to integrate automated detection methods into SWFSC's acoustic marine mammal monitoring protocol and this work is an important step in doing so.

\section{2:15}

2pAB5. Tracking fin and blue whales above the Juan de Fuca Ridge with a local seafloor seismic network. William S. D. Wilcock, Dax C. Soule (School of Oceanogr., Univ. of Washington, Box 357940, Seattle, WA 98195, wilcock@u.washington.edu), and Richard E. Thomson (Inst. of Ocean Sci., Sidney, BC, V8L 4B2 Canada)

The Endeavour segment of the Juan de Fuca mid-ocean ridge hosts several high-temperature hydrothermal fields. Previous analysis of bioacoustical data shows that zooplankton are enhanced at all depths above the hydrothermal vent fields compared with sites $\geqslant 10 \mathrm{~km}$ away. From 2003-2006, a seafloor seismic network was deployed around the hydrothermal vent fields to monitor earthquakes and it also recorded an extensive data set of fin and blue whale calls. As part of an investigation of a potential correlation between whale tracks, enhanced zooplankton concentrations, and hydrothermal vents above the Juan de Fuca Ridge, an automatic algorithm is being developed to track vocalizing whales that swim near the network. Events are detected by triggering with the ratio of short-term to long-term running RMS averages and whale calls are distinguished from earthquakes based on their spectra. For fin whales each 1-s arrival is identified based on its instantaneous amplitude and frequency and a pick is made at the midenergy point. A grid search method is used to localize calls using direct and multipath arrivals. The algorithm and preliminary results will be presented. [The Keck Foundation supported the seismic network and the Office of Naval Research is supporting this study.]

\section{$2: 30$}

2pAB6. Frequency quantiles and dual harmonic tracking for detection and classification of killer whale calls. Val Veirs (The Whale Museum, Friday Harbor, WA 98250, Colorado College, Colorado Springs, CO, val @ beamreach.org)

The Salish Sea Hydrophone Network, www.OrcaSound.net, is operating five streaming hydrophones in the critical habitat of the endangered Southern Resident orcas. Software is being developed that allows each site to sift through the approximately 10 Gbytes of information that are produced each day at each site in order to detect killer whale calls and related sounds. Detection of calls is accomplished via triggering on three time dependent features extracted in real-time from sequential power spectra. One of these three features is derived from the bandwidth between quartiles of the filtered power spectrum. The other two quantify the degree to which the power spectrum has one or two harmonic structures. Features are extracted into feature sets that save the details of each feature: amplitudes; durations; and time variations. A metric has been devised that measures the similarity of different sequences of feature sets. With this metric an unsupervised learning algorithm builds a tree structure that classifies sounds by their similarity. As new calls come in, they either fall into pre-existing groups or create new branches. Automatic reports are made via the Web and e-mail.

\section{2:45}

2pAB7. Tracking multiple sperm whales with widely spaced bottom-mounted hydrophones. Eva-Marie Nosal (Dept. of Ocean and Resources Eng., Univ. of Hawaii, 2540 Dole St., Honolulu, HI 96816)

One dataset from the 2nd Int. Workshop on Detection and Localization of Marine Mammals Using Passive Acoustics Data featured multiple clicking sperm whales recorded for $20 \mathrm{~min}$. Data were recorded on five widely spaced bottom-mounted hydrophones at the Atlantic Undersea Test and Evaluation Center. This dataset is used to develop and apply a model-based time difference of arrival (TDOA) method that is capable of simultaneously tracking multiple animals. Clicks on all hydrophones are detected. For every receiver pair, all possible time-differences of arrival (within reasonable time limits) are formed from the detected clicks. A scatterplot of TDOAs versus time, although noisy due to many false TDOAs formed from incorrectly associated clicks, reveals clear "tracks" of slowly varying TDOAs. These tracks are extracted and input to a TDOA tracking algorithm that gives 3-D likelihood surfaces of whale position. Local maxima in the likelihood surfaces are extracted and clustered to give estimated whale tracks.

\section{3:00-3:20 Break}

\section{Invited Paper}

2pAB8. The Acousonde: A miniature autonomous wideband recorder. William C. Burgess (Greeneridge Sci. Inc., 6060 Graham Hill Rd., Fl. 2 Stop F, Felton, CA 95018, burgess@ greeneridge.com)

The Acousonde ${ }^{\mathrm{TM}}$ is a newly designed miniature acoustic/ultrasonic recording tag incorporating several improvements over its predecessor, the Bioacoustic Probe. Design trade-offs for its acoustic data paths targeted acquisition of both near and distant odontocete echolocation clicks with minimal distortion while preserving general-purpose utility and low-power operation. Two acoustic channels are available: a low-power channel for long-term recording of signals up to $9 \mathrm{kHz}$ and a high-frequency channel for signals up to 100 $\mathrm{kHz}$. Each channel has its own dedicated hydrophone. For antialiasing, the low-power channel uses an adjustable switched-capacitor elliptic filter, while the high-frequency channel uses a fixed-frequency linear-phase filter; both filters may be bypassed if raw acquisition is desired. "Ping-pong" alternating sampling may be used to acquire samples from both channels concurrently, possibly to assess timeof-arrival differences between the two hydrophones. The controlling microprocessor, an ARM 9 with vector floating point accelerator, can digitally filter and downsample acoustic data during acquisition to reduce storage requirements. Other sensors include a 3D accelerometer, a 3D compass, a depth transducer, and a temperature monitor. Eight gigabytes of data storage are available, with data offload via a MicroUSB connector. Initial tests with captive animals are planned shortly. [Work supported by ONR.] 


\section{Contributed Papers}

3:40

2pAB9. Validated reef fish sound scans of passive acoustic monitors on Hawaiian coral reefs. Timothy C. Tricas and Kelly Boyle (Dept. of Zoology and Hawaii Inst. of Marine Biol., Univ. of Hawaii at Manoa, Honolulu, HI)

Monitoring of fish sounds on coral reefs is a valuable potential technique for fisheries managers to remotely assess local fish populations and their behaviors. However, the species identity of most fish sounds on shallow coral reefs is not known. We have deployed two ecological acoustic recorders (EARs) at $20 \mathrm{~m}$ deep on two shallow reefs on the island of Hawaii. In order to validate which fish species produce these acoustic behaviors we used closed circuit rebreather diving, which produces almost no ambient acoustic noise, to record video and sonic behaviors of fish near the recording sites. From the videos we have identified 37 putative acoustic species many of which produce multiple sounds that include behaviors associated with intra- and interspecific aggression, feeding, courtship, and spawning. Cluster analysis was used to identify acoustic clades among and within species. Acoustic waveform average templates were constructed for different species clades and used in XBAT to screen the long-term recordings from the EAR. The use of these species-specific sound templates confirms the potential to detect diel, lunar, and longer-term rhythmicity for several species. This technique also shows promise for characterizing seasonal and annual periods of activity of sounds associated with context-specific behaviors.

\section{3:55}

2pAB10. Beaked whale density estimation from single hydrophones by means of propagation modeling. Elizabeth T. Küsel, David Mellinger (NOAA/PMEL, Hatfield Marine Sci. Ctr., Oregon State Univ., Newport, OR 97365, elizabeth.kusel@noaa.gov), Len Thomas, Tiago A. Marques (Univ. of St. Andrews, Scotland), David J. Moretti, and Jessica Ward (Naval Undersea Warfare Ctr., Newport, RI 02841)

Passive acoustic sonar systems offer many advantages to the study of marine mammals. For density estimation studies, it is important to evaluate the probability of detecting an animal as a function of its distance from the receiving sensor. In this work, acoustic propagation modeling is used to estimate the transmission loss as a function of depth and range between a source whale and a single-hydrophone receiver. The computed transmission loss is compared to ambient noise levels and source level distributions to estimate the detection probability as a function of range. Results will be compared to beaked whale data recorded on bottom-mounted sensors in the Atlantic Undersea Test and Evaluation Center (AUTEC) in the Bahamas, where the location of clicks is relative to one hydrophone. Source level and beam pattern extracted from digital acoustic tags (DTags) applied to a sample of animals at the same location will also be used in the detection model, and beaked whale spatial density will be estimated. The detection probability function will provide a relevant comparison to the detection function derived empirically from the DTag data by Marques et al. [Marques et al., J. Acoust. Soc. Am. (submitted).]
4:10

2pAB11. Passive acoustic monitoring of fish activity in the Hawaiian Archipelago. Pollyanna Fisher-Pool, Marc O. Lammers (Joint Inst. for Marine and Atmospheric Res., NOAA-CRED, 1125B Ala Moana Blvd., Honolulu, HI 96814, Pollyanna.Fisher-Pool@noaa.gov), Kevin Wong, Russell E. Brainard (NOAA Fisheries, Honolulu, HI 96814), and Whitlow W. L. Au (Univ. of Hawaii, Kailua, HI 96734)

Fish are an important component of coral reef ecosystems and can be valuable indicators of ecological change on reef environments. Many species of fish produce sounds and are therefore well suited for passive acoustic monitoring. Since 2006, NOAA has been using Ecological Acoustic Recorders (EARs) to monitor coral reef habitats in the main and Northwestern Hawaiian Islands in an effort to develop multi-year time series of biological activity at these locations. Sixteen EARs are presently deployed on reef habitats ranging in depth from 2 to 23 meters. Preliminary results reveal that each site is characterized by a unique composition of acoustically active fish . Many sounds are also common at multiple sites. Different species show considerable variability in their diet and seasonal patterns of activity. The results indicate that long-term acoustic monitoring of fish at these sites is likely to yield useful information about changing patterns of fish presence, behavior, and relative abundance. These metrics can be used to gauge the relative stability of ecosystems and be related to physical, oceanographic, and anthropogenic variables affecting them.

\section{$4: 25$}

2pAB12. Using moored passive acoustic recorders to assess seasonal occurrence and movements of southern resident killer whales in the coastal waters of Washington State. M. Bradley Hanson, Candice K. Emmons (NOAA/NMFS/Northwest Fisheries Sci. Ctr., 2725 Montlake Blvd. E, Seattle, WA 98112, brad.hanson@noaa.gov), Jeffrey A. Nystuen (Univ. of Washington, Seattle, WA 98105-6698), and Marc O. Lammers (Oceanwide Sci. Inst., Honolulu, HI 96839)

Designating critical habitat is mandated for species listed under the U.S. Endangered Species Act. This task has only been partly accomplished for southern resident killer whales (SRKWs) because winter distribution is poorly understood due to a variety of factors limiting visual sightings within their known central California to northern British Columbia range. To capitalize on the unique vocal behavior of resident killer whales, including podspecific dialects, two types of acoustic recorders were deployed at strategic locations that span the Washington coast. Between 2005 and 2008 recorders were deployed in early winter for an average of 175.5 days at up to four sites. These functioned for an average of 114.8 days and collected a total of 47 SRKW detections. This exceeds the number of visual sightings during the same time period (15). Additionally, northern resident, transient, and offshore killer whales were recorded as well as Pacific white-sided dolphins, and humpback and sperm whales. SRKW were detected by both types of recorders and in all areas. Detections were made between Jan. and July with the majority of these detections in Mar., Apr., and May. This new information will be of key importance to managers in meeting recovery goals. 


\title{
Session 2pAO
}

\section{Acoustical Oceanography and Underwater Acoustics: Environmental Inferences in Inhomogeneous Ocean Environments I}

\author{
David P. Knobles, Chair \\ Applied Research Labs., Univ. of Texas at Austin, Austin, TX 78713
}

Chair's Introduction-1:00

\section{Invited Papers}

1:05

2pAO1. Environmental influences on low-frequency, shallow-water acoustic propagation and inversion. George V. Frisk (Dept. of Ocean Eng., Florida Atlantic Univ., 101 N. Beach Rd., Dania Beach, FL 33004, gfrisk@ seatech.fau.edu)

Historically, the seabed has been considered to play the dominant role in shallow-water acoustic propagation at low frequencies. As a result, propagation models have focused on the incorporation of accurate values of bottom properties, while inversion techniques have concentrated on the determination of geoacoustic properties of the seabed. In recent years, however, the assumption of a benign water column has increasingly come under scrutiny in addressing both the forward and inverse problems. This paper addresses these issues as they relate to a measurement and inversion methodology in which acoustic data are acquired on synthetic radial apertures that are created using a moving source/receiver configuration in concert with precision navigation. These data can be transformed into the horizontal wavenumber domain to obtain an estimate of the propagation characteristics of the waveguide; namely, its modal content. The modal spectra can then be inverted using a variety of techniques to determine estimates of the waveguide parameters, specifically the geoacoustic properties of the seabed. The issues associated with the successful implementation of this modal mapping method in a highly variable shallow-water environment are discussed. [Work supported by ONR.]

\section{$1: 25$}

2pA02. The impact of ocean sound speed variability on the uncertainty of geoacoustic parameter estimates. Ross Chapman and Yongmin Jiang (School of Earth and Ocean Sci. Univ. of Victoria, Victoria, BC V8W 5C2, Canada, chapman@uvic.ca)

This paper investigates the influence of water column variability on the estimates of geoacoustic model parameters obtained from matched field inversions. The acoustic data were collected on the New Jersey continental shelf during the Shallow Water 2006 experiments. The oceanographic variability was evident when the data were recorded. To quantify the uncertainties of the geoacoustic parameter estimates in this environment, Bayesian matched field geoacoustic inversion was applied to multi-tonal continuous wave data. The spatially and temporally varying water column sound speed was parametrized in terms of empirical orthogonal functions and included in the inversion. Its impact on the geometric and geoacoustic parameter estimates was then analyzed by the inter-parameter correlations. Two different approaches were used to obtain information about the variation of the water sound speed. One used only the profiles collected along the experimental track during the experiment, and the other also included observations from a larger area and a greater time period. The geoacoustic estimates from both the large and small sample sets were consistent. However, due to the diversity of the oceanic sound speed, more empirical orthogonal functions were needed in the inversion when more sound speed profile samples are used. [Work supported by ONR.]

\section{$1: 45$}

2pAO3. Observed temporal statistics of acoustic travel time and intensity in the South China Sea. Ching-Sang Chiu and Christopher W. Miller, D. Benjamin Reeder, Justin M. Reeves, Steven R. Ramp (Dept. of Oceanography, Naval Postgraduate School, 833 Dyer Rd., Monterey, CA 93943, chiu@nps.edu)

The coupled ocean and acoustic variability in the Northeastern South China Sea basin was monitored using moored oceanographic and acoustic sensors during the 2005-2006 Windy Island Soliton Experiment (WISE). The temperature and current records captured prominent ocean variability at multiple scales, induced by mesoscale eddies, internal tides, and large amplitude internal waves. The latter two were transbasin, propagating from near the Luzon Strait, through the deep basin, onto the northeastern shelf. The concurrent acoustic measurements were attained from the transmission of a $400 \mathrm{~Hz}$, phase-modulated signal along a $166 \mathrm{~km}$ path every $15 \mathrm{~min}$. The receptions were processed to give the arrival structure of a pulse and its temporal change over a nine-month period. The observed temporal variability in the statistics of the acoustic travel time and intensity were analyzed using time-series techniques and models with emphasis to elucidate the connection and sensitivity to the observed ocean variability. Results from the analysis are discussed, as they pertain to the ocean processes and those parameters that can potentially be inferred from these types of acoustic transmissions. [The research is sponsored by the Office of Naval Research.] 


\section{Contributed Papers}

\section{2:05}

2pA04. Phase front fluctuations due to internal waves in shallow water 2006 experiment. Boris Katsnelson (Voronezh State Univ., Voronezh, Russia), Mohsen Badiey, Jing Luo (Univ. of Delaware, Newark, DE 19716), and James Lynch (Woods Hole Oceanograph. Inst., Woods Hole, MA 02543)

Experimental results and theoretical analysis of the sound phase front fluctuations measured by Vertical and Horizontal Line (VHLA) array (or L-shaped array) are presented. Low frequency broadband signals were radiated by the source (Miami Sound Machine) placed at the distance of $\sim 25$ $\mathrm{km}$ from the VHLA. During the time period 18:00-22:00 GMT on Aug. 17, 2006, a train of internal solitons traveled across the acoustic track and initiated fluctuations of the sound field. In an earlier paper [J. Acoust. Soc. Am. 124, EL66-EL72 (2008)], it was shown that intensity of these fluctuations results from the horizontal refraction of the sound waves due to internal solitons. Here we show the acoustic phase front fluctuations for separate waveguide modes for the same period. To obtain phase fronts for the individual modes, a special approach using an L-shaped receiver array is used. The vertical part of the array was used to obtain modal amplitudes. Using the measured sound field based on the perturbation theory, modal phases at the HLA can be calculated. Results of data processing are compared with theoretical calculations. [Work supported by ONR, CRDF, and RFBR.]

\section{2:20}

2pA05. Signal intensity fluctuations in Shallow Water 2006 (SW06) experiment during Event 50. Mohsen Badiey, Jing Luo (College of Marine and Earth Stud., Univ. of Delaware, Newark, DE 19716), Boris Katsnelson (Voronezh State Univ., Voronezh, Russia), and James Lynch (Woods Hole Oceanograph. Inst., Woods Hole, MA 02543)

During the Shallow Water 2006 (SW06) experiment, several internal wave (IW) events were recorded by moving and stationary instruments simultaneously with the acoustic propagation tests. More than 50 IW events were recorded using the University of Delaware's R/V Sharp's radar. On this ship, a J15 sound source transmitted various acoustic signals at different bearings and ranges to the Woods Hole Oceanographic Institution's vertical and horizontal hydrophone arrays. During one of the IW events, the acoustic source was kept at a constant water depth while moving in horizontal plane with the advancing internal wave front. This internal wave packet, named Event 50, was also recorded by R/V oceanous radar a few kilometers away. The reason for placing the sound source on the advancing IW front was to examine the effects of azimuthal variability of the waveguide on the acoustic propagation. A detailed transition of the waveguide as the internal wave progressively occupied the acoustic propagation track between the source and receiver is reported. It is shown that the intensity peaks at small angles between the acoustic track and the internal wave front where sound intensity focusing and defocusing occurs. [Work supported by ONR 321OA and CRDF, and RFBR.]

\section{$2: 35$}

2pA06. Intensity measurements and fluctuations of acoustic transmissions from the Research Vessel Sharp during Shallow Water 2006. Georges A. Dossot, James H. Miller, Gopu R. Potty (Dept. of Ocean Eng., Univ. of Rhode Island, Narragansett Bay Campus, Narragansett, RI 02882), James F. Lynch, Aurthur E. Newhall (Woods Hole Oceanograph. Inst., Woods Hole, MA 02543), and Mohsen Badiey (Univ. of Delaware, Newark, DE 19716)

During the Shallow Water 2006 (SW06) experiment, the University of Delaware's R/V Sharp transmitted various acoustic signals at several different bearings and ranges to the Woods Hole Oceanographic Institute's vertical and horizontal hydrophone line array. The array was strategically positioned near the shelfbreak front, and in an area where internal waves are known to occur. During several of the R/V Sharp's acoustic transmissions, internal waves passed through the sound field. The internal waves and the shelfbreak front can cause complex multimode and multipath interference patterns which result in intensity variations of received acoustic signals. This presentation provides an overview of the R/V Sharp's transmissions, and the corresponding intensity fluctuations of received signals at the array. These fluctuations are compared to internal wave events that were recorded at both the transmission and reception locations. These internal wave events were also imaged by the R/V Sharp's radar and satellite-based radar. Following the work of Fredricks et al. [J. Acoust. Soc. Am. 117, 1038 (2005)], statistical distributions are fit to the calculated intensities for different transmission directions, distances, and times. These distributions are compared to modeled data with and without the internal wave field. [Work sponsored by the Office of Naval Research.]

\section{2:50}

2pAO7. Time-frequency pattern of the sound intensity fluctuations of midfrequency signals in presence of internal waves in Shallow Water 06 experiment. Boris Katsnelson, Valery Grigorev, and James Lynch (Woods Hole Oceanograph. Inst., 98 Water St., Woods Hole, MA 02543, jlynch @whoi.edu)

In given paper fluctuations of intensity of sound signals, radiated by the midfrequency source (R/V Knorr) during $\sim 5 \mathrm{~h}$ were studied. Broadband signals $(2-8 \mathrm{KHz})$ were received by four single hydrophones fixed at the bottom (SHRUs) placed at different distances from the source (from 4 to 12 $\mathrm{km})$. These four acoustic tracks have different directions relative direction of propagation of the train of intensive internal waves (the corresponding angles in horizontal plane are from $\sim 5$ to $\sim 15 \mathrm{deg}$ ). Time-frequency diagrams were constructed using frequency filtering of the spectrum of broadband signals with sliding narrow window. Temporal dependences of intensities of received signals within frequency window were constructed for period 14:00 until 19:00 GMT for experiment carried out on 13 August. During this time train of internal solitons was registered, propagating toward the coast. Mentioned time-frequency diagrams demonstrate specific features of influence of internal waves on the temporal variations of the sound intensities at four SHRUs. More exactly variations of predominating frequency in spectra correspond to variations of positions of the solitons at the acoustic tracks. Results of experimental data are compared with theoretical estimations. [Work was supported by RFBR and CRDF.]

\section{3:05}

2pA08. Acoustic ducting, refracting, and shadowing by curved nonlinear internal waves in shallow water. James F. Lynch, Ying-Tsong Lin, Timothy F. Duda, Arthur E. Newhall, and Glen Gawarkiewicz (Woods Hole Oceanograph. Inst., Woods Hole, MA 02543, jlynch@whoi.edu)

Nonlinear internal waves are common on continental shelves and have been shown to have strong effects on acoustic propagation and scattering. Transmissions from previous field work performed in an along-shelf geometry show very strong ducting of low-frequency $(50-500 \mathrm{~Hz})$ sound between nonlinear waves. This strong ducting effect is acoustically important. Most all of the acoustic propagation studies of internal waves have used straight line internal wave fronts for individual waves or packets. In our work, we implement a theoretical analysis using the Weinberg-Burridge horizontal rays and vertical modes formalism to study the acoustic ducting, refracting and shadowing of low-frequency sound due to curved internal waves, which seem to dominate in shelf break regions. A three-dimensional parabolicapproximation sound propagation model is also used with consideration of realistic environmental conditions, and the modeling results provide a clear depiction of the underlying physical processes. The oceanographic origin of the internal wave curvature is discussed, as this affects the predictability of the sound speed field. [This work is sponsored by the Office of Naval Research.] 


\title{
Session 2pBB
}

\section{Biomedical Ultrasound/Bioresponse to Vibration: Biomedical Applications of Standing Waves}

\author{
Armen Sarvazyan, Cochair \\ ARTANN Laboratories, 1459 Lower Ferry Rd., Trenton, NJ 08618 \\ Martin Wiklund, Cochair \\ Dept. of Applied Phyiscs, Royal Inst. of Tech., SE-10691, Stockholm, Sweden \\ Chair's Introduction-1:15 \\ Invited Papers
}

$1: 20$

2pBB1. Ultrasonic manipulation in microfluidic chips for accurate bioparticle handling. M. Wiklund, O. Manneberg, J. Svennebring, B. Vanherberghen, B. Onfelt, and H. M. Hertz (Dept. of Appl. Phys., Royal Inst. of Technol., SE-10691 Stockholm, Sweden)

Micro-manipulation of cells or other bioparticles has important applications in biological and biomedical research. Dielectrophoresis and optical tweezers are the classical tools in this field. Ultrasonic standing wave (USW) technology is presently emerging as powerful alternative, especially in microfluidic chips and other miniaturized systems. In this contribution we will review our present activities in USW-based particle manipulation in microfluidic chips. Recent experiments and simulations allow us to tailor the spatial distribution of the USW force field by multi-frequency actuation and appropriate microchannel/transducer resonance design. We introduce the concept of ultrasonic micro-cages for single-cell or single-particle 3-D ultrasonic manipulation. It is shown that these manipulation tools can be combined with high-resolution optical microscopy, thereby allowing state-of-the-art characterization of individual cells. Frequencymodulation is shown to stabilize the manipulation performance as well as allowing flow-free transport of particles and cells. Environmental control inside the channels has been achieved and proliferation and viability studies are promising. Finally, for bio-analysis, we demonstrate potential for femtomolar bio-analytical sensitivity in bead-based assays using USW enrichment.

\section{$1: 40$}

2pBB2. Acoustofluidics: Theory and simulation of streaming and radiation forces at ultrasound resonances in microfluidic devices. Henrik Bruus (Dept. of Micro- and Nanotechnology, Tech. Univ. of Denmark, DTU Nanotech, Bldg. 345 East, DK-2800 Kongens Lyngby, Denmark, Henrik.Bruus@nanotech.dtu.dk)

During the past few years, there has been an increasing interest in applying ultrasound waves to manipulate biological particles and liquids in microfluidic devices. To obtain optimized designs and functionalities of the acoustofluidic devices, more detailed theoretical studies and numerical simulations are called for. The basic second-order perturbation theory is presented for acoustic fields applied at ultrasound frequencies in silicon/glass systems containing water-filled microfluidic channels and chambers. For various specific device geometries, the resonance frequencies and corresponding modes of the acoustic fields are calculated numerically to first order. At these frequencies, the largest possible acoustic powers are obtained in the microfluidic system. The first order fields are then used as source terms in the equations for the time-averaged second order pressure and velocity fields, which are directly related to the acoustic radiation force on single particles and to the acoustic streaming of the liquid. For the radiation pressure effects, there is good agreement between theory and simulation, while the numeric results for the acoustic streaming effects are more problematic. Possible improvements in the latter case are discussed.

\section{2:00}

2pBB3. Transient processes in acoustic resonators. Armen Sarvazyan (Artann Labs., 1753 Linvale-Harbourton Rd., Lambertville, NJ 08530, armen@artannlabs.com) and Lev Ostrovsky (Zel Technologies/Univ. of Colorado, Boulder, CO 80305)

The use of ultrasound in resonators for manipulating particles in various biomedical applications is a relatively well studied topic. These studies were mostly concerned with steady-state processes. However, in certain important applications, such as stirring and mixing fluids in microfluidics and biosensors, the time scale of a process plays a crucial role. In this presentation we consider some of these applications. In particular, the radiation force-induced motion of microparticles and microbubbles in a swept-frequency ultrasonic resonator is considered. The particles are forced to move due to switching the resonance modes in a resonator cell, thus providing effective stirring of the fluid. Another field of medical application of standing waves, where transient processes are of crucial importance, is ultrasonic treatment of tissues. In this new field of ultrasonic therapy, standing waves are used for producing lesions in the tissue with simultaneous monitoring the formation of the lesion by automatic controlling parameters of the standing wave field. The spatial and temporal distribution of ultrasound energy in the resonator containing treated tissue portion is theoretically analyzed. Dynamics of temperature changes in the tissue, which is important for optimizing tissue treatment regimes, is evaluated. Experimental data illustrating theoretical results are presented. 
2pBB4. Current applications of ultrasonic resonators to biomolecular studies. Tigran Chalikian (Faculty of Pharmacy, Univ. of Toronto, 144 College St., Toronto, ON M5S 3M2, Canada, chalikan@phm.utoronto.ca)

The most versatile family of instruments providing the highest precision of acoustic measurements in the lowest sample volumes utilizes the method of fixed-path interferometer. The speed of sound in a medium is a simple function of its density and compressibility. Both parameters are determined by and, therefore, reflect the entire spectrum of intra- and intermolecular interactions within the system. In addition, compressibility probes the pressure response of these interactions. Current resonator-based instruments provide reliable measurements in diluted solutions of biological compounds with solute concentrations on the order of $1 \mathrm{mg} / \mathrm{mL}$ and solution volumes of $1 \mathrm{~mL}$. They require less than $1 \mathrm{mg}$ of sample, a range acceptable for the majority of biochemical and biophysical investigations. Ultrasonic velocimetric measurements have been employed to study a range of biologically relevant reactions, including the folding /unfolding transitions of proteins and helix-to-coil transitions of nucleic acids, as well as drug-DNA, ligand-protein, protein-protein, and protein-DNA association events. The limiting factor in such investigations is the need to rationalize the measured properties (volume, compressibility, and their derivatives) in terms of microscopic events, such as solute-solvent interactions. This presentation reviews biomolecular applications of acoustic measurements and discusses the development of approaches for microscopic interpretation of macroscopic volumetric observables.

\section{2:40}

2pBB5. Cylindrical standing wave resonator for liquid food quality control. Aba Priev (Hebrew Univ.-Hadassah Med. School, Jerusalem 91120; NDT Instruments Ltd., 56 Anilevich St., Jerusalem, Israel, abbap@ekmd.huji.ac.il) and Armen Sarvazyan (ARTANN Labs., Inc., Lambertville, NJ 08530)

In this paper, an innovative technology based on the use of ultrasonic cylindrical standing waves for continuous monitoring of quality of various liquid food products, such as milk, juices, beer, wine, and drinking water is described. A proprietary unique feature of the developed ultrasonic analyzer is that it employs a combined mode of operation using both high-intensity and low-frequency (10 $\mathrm{W} / \mathrm{cm}^{2}, 1 \mathrm{MHz}$ ) waves for separation and concentration of the high-molecular-weight particles (fat globules or cells) and low-intensity and high-frequency $\left(0.5 \mathrm{~W} / \mathrm{cm}^{2}, 10 \mathrm{MHz}\right.$ ) waves for compositional analysis. High accuracy for ultrasound velocity measurements (up to $0.001 \%$ ) and ultrasound attenuation (of about $1 \%$ ) and rapid testing time (2-20 s) have been achieved. Comparative analyses of the ultrasonic method with standard reference techniques have produced linear calibration curves for major components with correlation coefficients higher than 0.95 . It is thus possible to monitor total protein and fat content, and somatic cell count in raw milk in cowsheds, or salinity, turbidity, specific gravity, and particles (bacteria) in drinking water directly. Advantages of the proposed technology include the reagent-free nature, no need for sample pretreatment, ease-of-use, and low cost.

\section{3:00-3:30 Break}

\section{Contributed Papers}

\section{3:30}

2pBB6. Movement of liquids and solid particles on multiwall acoustic plates. Jeremy J. Hawkes (School of Chem. Eng. and Analytical Sci., The Univ. of Manchester, MIB 131 Princess St., Manchester, M1 7DN, UK), Rito Mijarez-Castro (Instituto de Investigaciones Elctricas, Morelos, Mexico, rmijarez@iie.org.mx), and Peter R. Fielden (The Univ. of Manchester, Manchester, M1 7DN, UK)

Well-defined vibration modes are used to form acoustic wells on flat plates. Within these dynamic traps, acoustic streaming continually stirs the contents. Traps can be used to hold liquids in separate heaps on a surface or to hold particles in levitated arrays. A protocol for creating trap patterns will be described, where FE modelling is used to select modes and then maneuver the modes to the desired frequency.

\section{$3: 45$}

2pBB7. Nonlinear ultrasonic standing waves in bubbly liquid: A numerical study. Vanhille Christian (Escet, Univ. Rey Juan Carlos, Tulipán s/n, 28933 Móstoles, Madrid, Spain, christian.vanhille@urjc.es) and Campos-Pozuelo Cleofé (Inst. de Acústica, CSIC, 28006 Madrid, Spain)

This study deals with the behavior of nonlinear ultrasonic standing waves in a bubbly liquid. A water air mixture is considered. The SNOW-BL numerical code gives the solution of a differential system coupling the nonlinear bubble equation with the linear wave equation. Several kinds of high power standing waves which may be useful in biomedical applications are considered here: pulses propagating in water with air bubbles clouds or layers, harmonic waves in a bubbly resonator, non-resonant standing waves in a fluid with bubbles. Conclusions about acoustic pressure distributions, waveforms, and frequency content, as well as mean and rms pressures, are given. Particular acoustical effects induced by the dynamics of the air- bubbles on the pressure standing wave are commented. Comparisons with results in homogeneous fluids are also presented. [Work supported by DPI2008-01429.]

\section{4:00}

2pBB8. Use of resonator method for body fluids composition assessment. Viktor Klemin (BIOM, 3 Veterinarnaya Str., Nizhny Novgorod, 603098, Russia, klemina@rf.unn.ru)

Ultrasonic resonator method of body fluids composition evaluation is described. The experimental data on assessment of whole blood, blood serum, gastric juice, and saliva are presented. The developed method is based on the assumption of additivity of the contributions of major components of fluids in their acoustic characteristics. The acoustic characteristics measured by the ultrasonic resonator method include the speed and absorption of ultrasound and their frequency and temperature dependences. The device for evaluation of body fluid composition comprises differential ultrasonic resonator cells with the volume of $80 \mathrm{mkl}$ each, operating at about $8 \mathrm{MHz}$, mounted in a micro-thermostat, and a compact electronic unit. The method allows for simultaneous determination of total protein, $\mathrm{Na}+$ and $\mathrm{K}+$, in saliva and gastric juice, lipid components (cholesterol total, cholesterol of high and low density, and triglycerides), the total protein and protein fractions in blood serum within a very short period of time, on the order of minutes, in contrast to conventional time consuming tests. Other advantages of the proposed technology include the reagent-free nature, no need for sample pretreatment, ease-of-use, and low cost. The accuracy of ultrasonic method for body fluid composition assessment is comparable to that of conventional time-consuming and expensive assays. 


\section{4:15}

2pBB9. Ultrasound standing wave fields control the spatial distribution of cells and protein in three-dimensional engineered tissue. Kelley A. Garvin (Dept. of Biomedical Eng., 205 Goergen Hall, Univ. of Rochester, Rochester, NY 14627, garvin@bme.rochester.edu), Denise C. Hocking, and Diane Dalecki (Univ. of Rochester, Rochester, NY 14627)

The application of ultrasound standing wave fields (USWFs) to cell suspensions results in the radiation force-mediated movement of cells to areas of the field separated by half-wavelength intervals. In this study, USWFinduced cellular arrangements were maintained after removal of the sound field by polymerization of a collagen type-I solution around the organized cell bands. Using a water tank setup, cell suspensions of varying concentrations were exposed to a 1-MHz, continuous wave USWF for $15 \mathrm{~min}$, during which time collagen polymerization occurred. An acoustic pressure amplitude of $0.2 \mathrm{MPa}$ was used to achieve the USWF-induced cell organization. Compared to sham samples with a random cell distribution, cell viability was not adversely affected by USWF exposure. The organization of cells into a banded pattern within the collagen gels resulted in a significant twofold increase in cell-mediated gel contraction, suggesting that USWFinduced cell organization leads to differential extracellular matrix remodeling. Protein organization within the tissue constructs was further controlled by USWF-mediated collocalization of soluble fibronectin to cell bands. These technologies have applications to the fabrication of tissue analogs with desired tissue characteristics for the repair or replacement of diseased or injured tissues and organs in the field of tissue engineering.

\section{4:30}

2pBB10. Two-dimensional numerical simulations for the time-averaged acoustic forces acting on a rigid particle of arbitrary shape in a standing wave. Jingtao Wang (Ctr. of Mech., Dept. of Mech. and Process Eng., ETH Zurich, 8092 Zurich, Switzerland, wang @imes.mavt.ethz.ch) and Jurg Dual (ETH Zurich, 8092 Zurich, Switzerland)

The time-averaged acoustic force can be applied to many practical fields such as contactless particle manipulation in biomedicine. It is necessary to accurately predict the mean forces on suspended obstacles to design ultrasonic particle manipulators. Although there have been many analytical solutions on this topic, it is difficult to determine the acoustic forces on obstacles under more complex system conditions such as proximity to the chamber wall, complex viscous function, acoustic streaming, and complicated particle shapes. Therefore, the numerical modeling may become a powerful tool. In this paper, the time-averaged forces, which act on rigid 2-D particles with different shapes in ideal and viscous fluids exerted by a standing sound wave field, are computed by solving the Navier-Stokes equations directly using the finite volume method (FVM) technique. The cylinder results agree well with Haydock's theoretical prediction and his lattice Boltzmann simulations. Then, the force and torque acting on a needle shaped particle in a standing wave are calculated and discussed in detail. Furthermore, the viscous effects of the host medium are also investigated. Our program with the FVM algorithm proves to be quite suitable for calculating the acoustic forces in standing waves.

\section{4:45}

2pBB11. Time reversal of monochromatic signals in elastic media. Brian E. Anderson, Robert A. Guyer, Timothy J. Ulrich, and Paul A. Johnson (Geophys. Group EES-17, Los Alamos Natl. Lab., Los Alamos, NM 87545)

A set of experiments has been conducted to show that time reversal of steady state monochromatic signals can produce spatial focusing in a reverberant elastic cavity when multiple channels are used. The transient portion of the received signals is not used. A single channel does not produce spatial focusing as it only drives the system according to its modal distribution. The amplitude of the energy at the focal location increases as the square of the number of channels used, while the amplitude elsewhere in the medium increases proportionally with the number of channels used. This work has importance in the field of medical ultrasound where the use of a long duration monochromatic excitation may be used for lithotripsy or other ultrasonic therapy. [This work is supported by Institutional Support (LDRD) at Los Alamos National Laboratory.]

TUESDAY AFTERNOON, 19 MAY 2009

COUNCIL SUITE, 1:00 TO 3:30 P.M.

\title{
Session 2pEAa
}

\section{Engineering Acoustics: Emerging Transduction Devices}

\author{
Fletcher Blackmon, Chair \\ Naval Sea Systems Command Division Newport, Newport, RI 02841
}

\section{Contributed Papers}

\section{1:00}

2pEAa1. The piezoelectric thickness mode hydrophone using tonpilz structure for deep-sea application. Min Sung, Haksue Lee, and Wonkyu Moon (Dept. of Mech. Eng., Pohang Univ. of Sci. and Tech., PIRO, Rm. 416, san31 hyja-dong, nam-gu, Pohang-si, kyungsangbuk-do, Korea)

A piezoelectric thickness mode hydrophone for deep-sea application using tonpilz structure was designed, and its macroprototype was made as a preliminary for the micromachined version. Several types of micromachined thin-diaphragm sensors for hydrophone application have been reported. They have two issues in deep-sea applications. One is a sensitivity problem. To enhance the sensitivity, a cone structure for sound pressure amplification was placed between the clamped diaphragm and the piezoelectric square plate. To evaluate the effect of the cone structure on sensitivity, which was motivated by tonpilz structure, an air-backed prototype was made using aluminum and piezoelectric ceramic square plate of $700 \mu \mathrm{m}$ length and $400 \mu \mathrm{m}$ thickness. A lumped parameter model was used in the design procedure. The measured sensitivity of the prototype was $-249.60 \mathrm{~dB}$ ref. One volt per mi- cropascal without a preamplifier, and the measured resonant frequency was $30 \mathrm{kHz}$, which agreed with the prediction. The other is an endurance problem due to high hydrostatic pressure. To develop the endurance for the high hydrostatic pressure in deep-sea, PZT thickness mode and oil-backing on the diaphragm backside were proposed. The comparison of oil-backing and airbacking is to be presented as an evaluation of the endurance development. [Research supported by MRCnd.]

\section{$1: 15$}

2pEAa2. Rapid identification of candidate materials for tonpilz head-mass design. Scott P. Porter, Douglas C. Markley, David J. Van Tol, and Richard J. Meyer, Jr (Appl. Res. Lab., The Pennsylvania State Univ., P. O. Box 30, State College, PA 16804, scott.porter@psu.edu)

Optimum performance of a tonpilz transducer head-mass section is often achieved when the mass is minimized and the stiffness is maximized. High stiffness keeps head-mass modes away from the band of interest with minimal material; low mass maximizes the motor section aspect ratio and re- 
duces the stored energy in the head-mass, raising the electromechanical coupling coefficient. Therefore, the material selected for the head-mass construction plays an integral role in transducer performance. In the practical design of tonpilz radiating heads, a tool which can rapidly identify the materials best suited for a given design is desirable. The figure of merit, $\mathrm{E} \rho^{3}$, has traditionally been used. This figure of merit, however, is based on thinplate approximations. Most actual tonpilz head-masses require thick-plate theory for accurate analysis. In this paper, the authors use a numerical routine to rapidly assess the thick-plate behavior of various materials and rank their suitability for a given head-mass geometry. These results are corroborated with finite element modeling. Finally, the thick-plate ranking scheme is compared to the traditional figure of merit. The results of this comparison challenge established assumptions about the best materials for head-mass design and identify ceramic materials as strong candidates for future designs.

\section{$1: 30$}

2pEAa3. Investigation of single crystals for U.S. Navy standard electro-acoustic transducer applications. Jeffrey A. Szelag and Thomas R Howarth (Naval Sea Systems Command Div. Newport, Newport, RI)

The Underwater Sound Reference Division (USRD), Naval Sea Systems Division in Newport, RI, is the U.S. Navy's primary source for the provision of standard underwater electro-acoustic reference transducers. USRD has over 40 years of traceable and repeatable historic data for each transducer that is currently available for underwater calibrations and experiments. As such, USRD's primary focus is on the continuous development of new reference transducers incorporating the latest technologies available. Requirements for these standards include repeatability over extended time, traceability, and ease of maintenance. This presentation covers an on-going USRD investigation to feature single crystal PMN-PT as the active substrate in the current F41 program transducer. In addition, lossy rubber substrates are being examined to replace the legacy corprene passive materials in order to extend the operating depths of the transducer. Our presentation will begin with an overview of the fabrication techniques followed by experimental underwater data comparing the new active and passive materials with the traditional F41.

\section{$1: 45$}

2pEAa4. A generalized differential effective medium model of piezoelectret foams. Michael R. Haberman (Appl. Res. Labs., The Univ. of Texas at Austin, 10000 Burnet Rd., Austin, TX 78758)

Piezoelectrets are closed-cell foams consisting of a continuous polymer matrix containing preferentially oriented and electrically polarized ellipsoidal voids. These materials display low density, low characteristic impedance, thicknesses on the order of $100 \mu \mathrm{m}$, and potential to act as conformal transducers on complex geometries. They therefore provide an intriguing alternative to piezoelectric ceramics and piezopolymers for applications such as air-coupled ultrasonics or underwater receive elements. Unfortunately, some fundamental attributes of piezoelectret behavior, such as their nonmonotonic change in receive sensitivity with increasing ambient pressure, remain unexplained by physical modeling or experiment. To explain this and other behavior, this work develops a fully coupled multiscale microelectromechanical model which captures the void-scale physics and predicts macroscopically observed piezoelectric properties. The model employs volume averaged Green's function solutions of the three-dimensional stress equilibrium equations and Gauss's Law. The resulting model approximates the effective stiffness, dielectric permittivity, and piezoelectric coupling tensors as a function of the constituent material properties, void fraction, shape, and orientation, as well as the deposited charge density. Strain localization tensors of the model are then employed to approximate piezoelectret dependence and external pressure. Model results show good agreement with experimental results and data from the literature.

\section{2:00}

2pEAa5. A micromachined piezoelectric flexural mode hydrophone for audible frequency application. Haksue Lee, Sungjoon Choi, and Wonkyu Moon (Dept. of Mech. Eng., Postech, South Korea, wkmoon@postech.ac .kr)
A micromachined hydrophone is designed for audible frequency application $(20 \mathrm{~Hz}-20 \mathrm{kHz})$. The basic structure is a flexural unimorph consisting of a clamped silicon diaphragm and a piezoelectric film. Two design approaches can enhance its sensitivity. One is a unimorph with unequal radii between the piezoelectric film and the diaphragm, which increases electromechanical coupling. The other is an air-backed chamber, which reduces mechanical impedance in the flexural vibration and, consequently, enhances sensitivity. To predict the effects of these approaches on sensitivity, an equivalent transduction model is composed and used for calculations. In the case of oil backing, compared to air backing, a reduction in sensitivity of about $40 \mathrm{~dB}$ is expected. The dimensions of the micromachined diaphragm (piezoelectric film) are $630 \mu \mathrm{m}(400 \mu \mathrm{m})$ radius and $15 \mu \mathrm{m}(3 \mu \mathrm{m})$ thick. A fabricated microsensor is constructed as a hydrophone by using a rubber housing filled with castor oil. The measured sensitivity is $-228.3 \mathrm{~dB}$ at $1 \mathrm{~V}$ $/ \mu \mathrm{Pa}$ without a preamplifier. A flat frequency response within $\pm 1 \mathrm{~dB}$ deviations were measured in the $100 \mathrm{~Hz}-5 \mathrm{kHz}$ band. Based on the simulation, it can resist the hydrostatic pressure of $100 \mathrm{~m}$ depth, without pressure balancing, while decreasing in sensitivity. The results of a larger model having a higher sensitivity of $-207.6 \mathrm{~dB}$ will also be presented. [Research supported by MRCND and R0A-2007-000-20042-0.]

\section{2:15}

2pEAa6. Advantages of piezoelectric microelectromechanical systems (MEMS) microphones. Robert Littrell and Karl Grosh (2250 GG Brown Bldg., 2350 Hayward St., Ann Arbor, MI 48109)

Microphones fabricated using microelectromechanical systems (MEMS) technology are one of the fastest growing applications of MEMS. Capacitive sensing has been the dominant form of transduction in both traditional and recently commercialized MEMS microphones. Models and experiments, however, indicate that the thin layers and fine spatial resolution made possible by MEMS technology lend themselves more appropriately to piezoelectric microphones. Although piezoelectric MEMS microphones have typically been shown to have a relatively high noise floor, this limitation can be overcome with appropriate design and high quality piezoelectric material. Models indicate that piezoelectric MEMS microphones can achieve a comparable noise floor to capacitive MEMS microphones of similar size and bandwidth while achieving 1000 times greater dynamic range. A piezoelectric MEMS microphone utilizing aluminum nitride (AIN) will be presented. Previously experienced film quality issues have been addressed. This microphone is designed to have a noise floor below $40 \mathrm{~dB}$ (A), a dynamic range greater than $160 \mathrm{~dB}$ SPL, a $10 \mathrm{kHz}$ bandwidth, and a sensing area of less than $1 \mathrm{~mm}^{2}$.

\section{2:30}

2pEAa7. Infrasonic microphone. Timothy Marston and Thomas B. Gabrielson (Grad. Prog. in Acoust., Penn State Univ., PO Box 30, State College, PA 16804,tmm357@psu.edu)

The high cost of many infrasonic transducers can be a setback for universities interested in performing research in the field of infrasound. Reasonably effective infrasonic transducers can be constructed in the laboratory, however, and for a fraction of the cost. A simple, inexpensive infrasonic microphone that operates on the principle of carrier demodulation has been constructed. Noise analysis of the microphone demonstrates its potential usefulness for cost-effective field research and deployment in temporary infrasonic arrays. [Research funded by the Penn State Applied Research Laboratory Educational and Foundational Fund.]

\section{$2: 45$}

2pEAa8. Mechanical characterization of capacitor microphones for analysis and study. YuFan Chuang (Master Program of Electroacoustics, Feng Chia Univ., 100 Wenhwa Rd., Seatwen, Taichung, Taiwan 40724, ROC, yufan420@gmail.com), S. J. Pawar, and Jin H Huang (Feng Chia Univ., Taichung, Taiwan 40724, R.O.C.)

An attempt is made to investigate the mechanical properties of the diaphragm of capacitor microphone using MTS Tytron 250 micro-force testing machine. The compliance of the diaphragm is calculated from the forcedisplacement diagram. The simulation of the microphone is carried out using equivalent circuit method. The mechanical parameters like mass 
(measured), compliance (measured and calculated), and resistance (assumed from literature) are being used to formulate mechanical domain of microphone in equivalent circuit model. The sensitivity curve of microphone is being plotted. Simultaneously, the experimental measurement has been carried by PULSE Electroacoustics with the software Sound Check in an anechoic chamber. The simulation and experimental results for sensitivity are found to follow each other within a wide frequency range. The characteristic investigation is carried out using validated equivalent circuit model for parametric study of microphone. [Work supported by Contract Nos. NSC 95-2221-E-035-013-MY3 and 95-2221-E-035-087. The authors would like to thank Leo Liao and Jack Wei of Merry Electronics Co. Taiwan and Listen Inc. for supplying Sound Check measurement.]

\section{3:00}

2pEAa9. Head related transfer functions for KEMAR. Gunnar Rasmussen (G.R.A.S. Sound \& Vib., Skovlytoften 33, 2840 Holte, Denmark,gr@gras.dk)

The head related transfer functions (HRTFs) for KEMAR for a number of ear pinnas of different sizes and hardnesses have been measured. Right and left ear differences on KEMAR as well as change in HRTFs over the last 40 years will be reported along with data for the ITU - Type 3 and the IEC 60959 standardized pinna. The influencing factors on the uncertainty budget will be discussed.

\section{$3: 15$}

2pEAa10. A transfer matrix method for estimating the dispersion and attenuation of plane waves in the standing wave tube. Kang Hou and $\mathrm{J}$. Stuart Bolton (Herrick Lab, Purdue Univ., West Lafayette, IN)

In this paper, an iterative method based on the transfer matrix approach is described for evaluating the sound speed and attenuation constant of air in a standing wave tube. The procedure is based on transfer matrix, the fourmicrophone method for measuring the transmission loss of acoustical materials. In the latter procedure, a sample is placed between two pairs of microphones, where the sample is exposed to an incident plane wave field. In the present case, the air inside the standing wave tube is treated as the sampled material. Since the knowledge of the dispersion characteristics of plane waves within the tube is required to perform this measurement, an iterative method must be applied at the postprocessing step to estimate the complex wave numbers in the sample section. The result from the experiments showed that the air temperature within the tube has a significant impact on the results. The measured results were found to be in good agreement with the Temkin formula for attenuation in tubes. In addition, the approach may be extended to the accurate measurement of viscosity and sound velocity of liquids and gases.

TUESDAY AFTERNOON, 19 MAY 2009

COUNCIL SUITE, 3:30 TO 4:40 P.M.

\title{
Session 2pEAb
}

\section{Engineering Acoustics: Piezoelectric Energy Harvesting}

\author{
Thomas R. Howarth, Chair \\ Naval Sea Systems Command Division Newport, Newport, RI 02841
}

Chair's Introduction-3:30

Invited Papers

$3: 35$

2pEAb1. Bridging the gap between macro- and microdevices for manufacture of piezoelectric energy harvesters. Arthur L. Chait (EoPlex Technologies, Inc., 3698-A Haven Ave., Redwood City, CA 94063, achait@eoplex.com)

In this presentation, materials engineer and entrepreneur, Arthur L. Chait will show why there is a gap at the miniature scale when building complex devices with multiple materials. He will explain why few technologies can handle several materials, with complex geometries, in small parts. Miniature piezoelectric (PZ) energy harvesters are an example of this challenge. Mr. Chait will describe his piezoelectric energy harvester, designed for tire pressure sensor systems. This tiny device uses PZ materials to convert some of the vibration of the tire into electricity. The electric current is stored in a capacitor and used to power the system. Audiences will learn why it is difficult and costly to build a small, rugged system to power a tire sensor. The anatomy of this device includes at many diverse materials and five basic design elements. These are bonded into a strong package about the size of a contact lens. Building this energy harvester would be almost impossible with conventional manufacturing techniques. Mr. Chait will share a relatively new solution; i.e., a print deposition technique that produces thousands of parts simultaneously from many different materials. Active elements like circuits, catalyst beds, mixing chambers, capacitors, and piezoelectric actuators are produced all in one step.

\section{4:00}

2pEAb2. Acoustic energy harvesting using an electromechancal Helmholtz resonator. Fei Liu (Dept. of Mech. and Aerosp. Eng., Univ. of Florida, Gainesville, FL 32611-6250), Alex Phipps, Stephen Horowitz, Louis Cattafesta, Toshikazu Nishida , and Mark Sheplak (Univ. of Florida, Gainesville, FL 32611-6250)

This talk presents the development of an acoustic energy harvester using an electromechanical Helmholtz resonator (EMHR). The EMHR consists of an orifice, cavity, and a piezoelectric diaphragm. When the acoustic wave is incident on the EMHR, a portion of acoustic energy is converted to electrical energy via piezoelectric transduction in the diaphragm of the EMHR. Moreover, the diaphragm is coupled with energy reclamation circuitry to increase the efficiency of the energy conversion. Two power converter topologies are 
adopted to demonstrate the feasibility of acoustic energy reclamation using an EMHR. The first is comprised of only a rectifier, and the second uses a rectifier connected to a flyback converter to improve load matching. Experimental results indicate that approximately 30 $\mathrm{mW}$ of output power is harvested for an incident sound pressure level of $160 \mathrm{~dB}$ with a flyback converter. Such power level is sufficient to power a variety of low power electronic devices.

\title{
Contributed Paper
}

\section{4:25}

2pEAb3. Theoretical study of thermoacoustic power conversion with a piezoelectric transducer. Carl Jensen and Richard Raspet (Natl. Ctr. for Physical Acoust., Univ. of MS, Univ., MS 38677)

A heat-driven thermoacoustic engine prototype has been designed with a piezoelectric transducer as an electroacoustic transformer. The design under consideration uses a bending, unimorph transducer placed between two identical thermoacoustic engines operating out of phase such that the trans- ducer is driven in a push-pull fashion. The thermoacoustic engine is modeled using a one-dimensional low amplitude simulation. The piezoelectric unimorph is modeled by an electroacoustic equivalent circuit aimed at capturing the first order resonant behavior of the transducer; loss mechanisms from hysteretic behavior of the dielectric or elastic properties are not considered by the model. Issues in optimizing the geometry of the model will be presented as well as comparisons to other forms of waste heat power harvesting such as thermoelectric.

TUESDAY AFTERNOON, 19 MAY 2009

STUDIO SUITE, 1:00 TO 4:00 P.M.

\section{Session 2pMUa}

\section{Musical Acoustics: Wind Instruments II}

\author{
R. Dean Ayers, Chair \\ 624 Valley View Dr., Medford, OR 97504-6356
}

Invited Papers

1:00

2pMUa1. Evaluation of two control parameters of trumpet players as function of sound features. Rene Causse (Ircam, 1 place Igor Stravinsky, F-75004 Paris, France) and Vincent Freour (McGill Univ., Montréal, PQ Canada.)

Experiments were performed by five trumpet players with the same trumpet and mouthpiece. For each subject, two control parameters are measured: mouth-pressure and force applied by the lips on the mouthpiece. The sound is also recorded. The players are asked to play sustained notes at different pitch and sound levels. We calculate fundamental frequency, sound pressure level, and some spectral features: noisiness, spectral roll-off, etc. Although little mouth-pressure variability as function of pitch and dynamic appears between subjects, clear differences were obtained in terms of the force applied on the mouthpiece. Therefore, we suggest to focus on the link between sound features and the ratio of these two control parameters measured, sort of "gesture estimator." The representation of the ratio as function of the sound features shows a good classification of musicians. Moreover, in some specific cases such as noisiness, the relation seems independent from the players. These observations suggest that these two control parameters could be efficient to characterize players' embouchure and to access some sound characteristics without knowledge of the mechanical characteristics of the lips. These results will be described and discussed, and direction for future works suggested. [Consonnes project, supported by the French Research Agency.]

\section{$1: 30$}

2pMUa2. Does a brass-instrument's timbre depend on the alloy from which it is made? Robert W. Pyle, Jr. (11 Holworthy Pl. Cambridge, MA 02138, rpyle@post.harvard.edu)

Most brass players with a few years experience would give an affirmative answer to the question posed in the title. In the past, audible differences have proven elusive when pursued through spectral analysis of sustained tones, perhaps because the right question was not asked. This talk will examine several attributes of trombone spectra. The trombone used has a "modular construction" so that different bells can easily be installed on an otherwise unchanged instrument. A clear difference, consistent with the player's and listener's perception of the tone quality, emerges between yellow brass $(70 \% \mathrm{Cu}, 30 \% \mathrm{Zn})$ and red brass $(90 \% \mathrm{Cu}, 10 \% \mathrm{Zn})$. Transient behavior, particularly during the attack, is at least as important to the performer as sustained sounds. Here, too, differences are attributed to the alloy. Yellow brass is characterized by "crisp" attacks, red brass by "rounded" attacks. This talk will also analyze attack transients, even though attacks that are perceived as similar appear quite variable when examined in detail. 
2pMUa3. Comparison of the mechanics of the brass players' lips during slurred note transients. John P. Chick and Shona M. Logie (SEE, Univ. of Edinburgh, Kings Bldgs., Edinburgh EH9 JL, UK, john.chick@ed.ac.uk)

When sounding a note on a brass instrument, a strong coupling is established between the vibrating lips of the player and the air column resonance. In moving between notes as smoothly as possible, or "slurring," there is a transition from one strongly coupled, steady-state, regime of the lip/air column to a different steady-state coupling of the lip/air column. To the listener, there are sometimes subtle differences in the sound of the transition between notes depending on how this transition is achieved: by lip slur, valve slurs, and, in the case of the trombone, slide slurs. This paper uses data collected from high speed video capture of the players' lips, synchronized with microphones in the mouthpiece and the bell of the instrument, to investigate and compare the motion of the brass players' lips for different types of slurred internote transients.

\section{2:30}

2pMUa4. Acoustical history of the tuba. Arnold Myers (Univ. of Edinburgh, Reid Concert Hall, Bristo Square, Edinburgh EH8 9AG, U.K.)

Low-pitched valved brass wind instruments are often described generically as tubas. In fact, the term "tuba" covers a broad family of instruments, with sounding lengths ranging from the $8 \mathrm{ft} \mathrm{C}$ of the classic French orchestral tuba to the $18 \mathrm{ft} \mathrm{Bb}$ of the brass band and orchestral contrabass. The first instrument designated as a tuba was introduced in 1835, and since then a variety of models differing in bore profile as well as in nominal pitch have been used. This paper explores the the historical development of the tuba family from an acoustical perspective, presenting and discussing measurements of bore profile and input impedance for a number of representative instruments from different periods and musical traditions.

\section{3:00-3:15 Break}

\section{Contributed Papers}

\section{3:15}

2pMUa5. The double bell descant euphonium. Frederick J. Young (Div. of Com. and Arts, Univ. of Pittsburgh, 800 Minard Run Rd., Bradford, PA)

A euphonium is presented that has better intonation and is easier to play in the extreme registers. It is a complete double horn having five double valves in contrast to the incomplete normal double French horn having only three double valves. The valves descend 2, 1, 3, 4, and 5 semitones from the open tones. The large bell is for the $\mathrm{B} b$ standard part of the instrument. A switch valve activates the smaller alto bell in the key of high $\mathrm{E} \nvdash$ and sends the air through the five shorter valve slides and out the small bell. The fundamental frequency of the $\mathrm{E}$ side is $82.4 \mathrm{~Hz}$ or six semitones above the fundamental of the $\mathrm{B} b$ euphonium. The key of $\mathrm{E}$ rather than $\mathrm{F}$ or $\mathrm{E} b$ enables all notes in the complete range (about $29-988 \mathrm{~Hz}$ ) to be played with a single tunable valve slide. Either of the other keys would introduce a need for one additional valve or compromise the intonation.

\section{3:30}

2pMUa6. Optimization of valve tube lengths for brass instruments. Frederick Young (Div. of Com. and Arts, Univ. of Pittsburgh, 800 Minard Run Rd., Bradford, PA 16701, youngfj@youngbros.com)

The intonation deviations from the well-tempered scale are presented for valve brass wind instruments. Compensating and full double instruments are considered. Systems including descending valves and less popular ascending valves are studied. The lengths of the valve tubes are optimized by minimizing the overall root mean squared (rms) intonation error using the method established in 1967 [R. Young, J. Acoust. Soc. Am. 42, 224-235 (1967)]. Open note detuning is considered in each case and improves the overall intonation. The worst three valve instrument without open note detuning has an rms deviation 24 cents The best of the three and four valve instruments is a three valve compensating euphonium tuned 3 cents sharp on the open notes and flat on valves 2,1 , and 3 by 3, 3, 10 cents, respectively. It exhibits only 2 cents rms deviation on single valves and less than 3 cents on combinations of valves. The comparisons also include several new three, four, and five valve systems. Included is a double horn with only two double valves and a switch valve.

\section{3:45}

2pMUa7. The input impedance of an alphorn including an Alexander mouthpiece. Frederick J. Young (Div. of Com. and Arts, Univ. of Pittsburgh, 800 Minard Run Rd., Bradford, PA 16701, youngfj@youngbros.com)

The alphorns built and sold by the late Joseph Littleton of Hammondsport, NY are used in this study. His dimensions of bore as a function of position are used in solution of the Webster equation for impedance solved numerically earlier [F. J. Young, Acustica, 10, 91-97 (1960)]. The musical notation for the frequencies of the impedance peaks are given below. Here the subscripts refer to the harmonic number. The fundamental, rarely used, is 13 cents flat from $A$ at $55 \mathrm{~Hz}$. The intonations errors for. $\mathrm{C}_{1}, \mathrm{C}_{2}, \mathrm{G}_{3}, \mathrm{C}_{4}, \mathrm{E}_{5}$, $\mathrm{G}_{6}, \mathrm{~B}_{7}^{\mathrm{b}}, \mathrm{C}_{8}, \mathrm{D}_{9}, \mathrm{E}_{10}, \mathrm{~F}_{11}, \mathrm{G}_{12}, \mathrm{~A}_{13}, \mathrm{~B}_{14}^{\mathrm{b}}, \mathrm{B}_{15}, \mathrm{C}_{16}, \mathrm{C}_{17}^{\#}$, and $\mathrm{D}_{18}$ are 287, 58, 195, $28,1.5,0.83,-28,-0.85,-2.63,1.5,40,14,-49,1,-3,16,24$ and 28 cents. 


\title{
Session 2pMUb
}

\section{Musical Acoustics: Wind Instruments III and Mini Concert}

\author{
D. Murray Campbell, Chair \\ School of Physics and Astronomy, Univ. of Edinburgh, Edinburgh EH9 3JZ, U.K.
}

\section{Invited Paper}

$4: 15$

2pMUb1. Imitating the human voice: The renaissance cornett and sackbut. Murray Campbell (Sch. of Phys. and Astron., Univ. of Edinburgh, Edinburgh EH9 3JZ, U.K., d.m.campbell@ed.ac.uk)

In the 16th century the lip-reed instruments most commonly used in sacred and chamber music were the cornett (Italian "cornetto") and the sackbut (Italian "trombone"). The sound ideal described by contemporary writers for these instruments was clearly distinguished from the ceremonial and often military splendor of the trumpet ensemble: the cornett and the sackbut were expected to produce dynamic levels and timbres comparable to those of human singers. The acoustical features, which make it much easier to approach this ideal on the renaissance instruments than on modern trumpets and trombones are explored through measurements on original instruments and modern reproductions.

4:45-5:30 Mini Concert

TUESDAY AFTERNOON, 19 MAY 2009

BROADWAY III/IV, 1:00 TO 4:55 P.M.

\section{Session 2pPA}

\section{Physical Acoustics and Biomedical Ultrasound/Bioresponse to Vibration: Numerical Methods for Weak Shock Propagation}

\author{
Andrew A. Piacsek, Chair \\ Dept. of Physics, Central Washington Univ., Ellensburg, WA 98926-7422
}

\section{Invited Papers}

1:00

2pPA1. Numerical modeling of weak shock propagation: Past, present, and future. Andrew Piacsek (Dept. of Phys., Central Washington Univ., 400 E. University Way, Ellensburg, WA 98926, piacsek@cwu.edu)

This presentation provides an overview of the subject of numerical modeling of weak shock propagation, with the aim of providing context for subsequent talks in this session. First is a brief history of analytical approaches to the problem of weak shocks in both one and two dimensions, emphasizing the characteristic physical behavior of the solutions. This leads to a discussion of the physical processes that must be modeled, including the Rankine-Hugoniot conditions at the shock interface, nonlinear steepening, and selfrefraction, refraction and diffraction due to an inhomogeneous medium, dissipation, relaxation, and heating of the medium. Next is a summary of paraxial numerical methods, specifically the NPE and KZK equations, and their recent applications, followed by a description of efforts to develop "wide-angle" versions of these codes. Computational challenges, and possible approaches, to extending these methods to three dimensions are also presented. Last, a brief introduction to new approaches to 3-D modeling will be given, along with a discussion of the applicability of computational improvements such as adaptive meshing and parallel processing.

\section{$1: 20$}

2pPA2. Numerical simulation of acoustical shock waves: Beyond the parabolic approximation. Franck Dagrau, Mathieu Renier, François Coulouvrat, and Regis Marchiano (Univ. Pierre et Marie Curie, Institut Jean Le Rond d'Alembert (UMR CNRS 7107), 4 place Jussieu, 75005 Paris, France)

The KZK equation is the reference equation for the modeling of finite amplitude diffraction effects. It has been applied with success to many applications in various domains. Nevertheless, the parabolic approximation underlying the KZK equation limits the validity of this one to narrow-angle propagation. A new formulation of the Kuznetsov equation enables us to go beyond the parabolic approximation for the diffraction effects in the homogeneous case, the parabolic approximation being now limited only to the heterogeneous perturbation which is one order of magnitude smaller. This method formalizes and generalizes to the weakly heterogeneous case the 
previous work of Christopher and Parker [J. Acoust. Soc. Am. 90, 488-499 (1991)]. Special attention is paid to the implementation of boundary conditions (such as absorbing or perfectly matched layers). Several validation tests will illustrate the potential of the method, such as nonlinear focusing or scattering. Applications to sonic boom propagation in a turbulent atmosphere will finally be discussed.

\section{$1: 40$}

2pPA3. Extension of the iterative nonlinear contrast source method to nonlinear media exhibiting tissue-like attenuation. Martin Verweij, Jacob Huijssen (Lab. of Electromagnetic Res., Fac. of Elec. Eng., Mathematics and Comput. Sci., Delft Univ. of Technol., Mekelweg 4, 2628 CD Delft, The Netherlands, m.d.verweij@tudelft.nl), and Nico de Jong (Erasmus Medical Ctr., Rotterdam, The Netherlands)

Recently, the Iterative Nonlinear Contrast Source (INCS) method has been developed to compute the pulsed acoustic pressure field in a nonlinear and lossless medium that extends over a very large, 3-D domain. This method solves the nonlinear wave equation by considering the nonlinear term as a distributed contrast source in a linear background medium. The full nonlinear wave field follows by convolving the background Green's function with successive estimates of the contrast source. It has been shown that by spatiotemporal filtering of the convolution factors, the method yields accurate field predictions even for a discretization approaching two points per smallest wavelength or period. The current paper discusses how attenuation, in particular tissue-like power law losses, may be incorporated into the method through the introduction of a causal compressibility relaxation function. The latter gives the background the desired loss behavior. Fields in nonlinear media with different power law losses are presented, as computed by the extended INCS method. Moreover, the ability of both the lossless and the extended INCS method to evaluate fields at the shock formation distance is shown. Especially the latter results show the significance of using a proper, tissue-like power law. [Work supported by STW and NCF.]

2:00

2pPA4. Weak shock propagation in the ocean and marine sediments. B. Edward McDonald (Acoust. Div. Naval Res. Lab, Washington DC 20375, mcdonald@ccs.nrl.navy.mil)

The nonlinear progressive wave equation (NPE) [B. E. McDonald and W. A. Kuperman, JASA 81, 1406 (1987)] was initially developed to model weak shock waves in a refracting medium (specifically, ocean acoustic convergence zones). It has been refined to include high-angle and two-way propagation. Inclusion of two-way propagation improves agreement with empirical power laws for peak pressure from an explosive as a function of range and explosive charge. The NPE is currently being used to examine weak shock propagation into marine sediments for studies related to mine countermeasures. An intriguing behavior results from the stress-strain relation for Hertzian granular media, in which stress is proportional to the $3 / 2$ power of strain rate. Numerical and analytic solutions with the Hertzian nonlinearity reveal its most nonlinear behavior (shock formation) near zero stress, whereas in a fluid, shock formation results from high stress. Numerical experiments are presented to determine whether shocks resulting from Hertzian nonlinearity can be observed with nominal values of frequency-linear attenuation common to granular media. [Work supported by the Office of Naval Research.]

\section{2:20}

2pPA5. Modeling weak shocks produced by high-intensity focused ultrasound. Vera A. Khokhlova (Ctr. for Industrial and Medical Ultrasound, Appl. Phys. Lab., Univ. of Washington, 1013 NE 40th St., Seattle, WA 98105/ Moscow State Univ., Moscow 119991, Russia), Olga V. Bessonova (Moscow State Univ., Moscow 119991, Russia), Michael S. Canney, Michael R. Bailey (Univ. of Washington, Seattle, WA 98105), Joshua E. Soneson (Food and Drug Administration, Silver Springs, MD 20993), and Lawrence A. Crum (Univ. of Washington, Seattle, WA 98105)

Shock waves of up to $100 \mathrm{MPa}$ may form at the focus of high-intensity focused ultrasound (HIFU) transducers at clinically reported in situ intensities of up to $30,000 \mathrm{~W} / \mathrm{cm}^{2}$. The heating due to shocks is sufficient to boil tissue in milliseconds, which dramatically alters the treatment. Quantification of enhanced heating from shocks is therefore critical to treatment planning. In this work, several approaches and temporal grids of different resolutions were used to simulate HIFU fields. Peak positive pressure, which determines the shock amplitude, and thus the heating rate were found to be the most sensitive to the parameters of the numerical scheme. Heating rates calculated in modeling and estimated using weak shock theory from the measured and modeled waveforms were compared. Time to boil measured in tissue phantoms and tissue was used as a metric of the heating efficiency of shocks. It is shown that the bandwidth limitations in the waveform measurements result in underestimation of the heat rates, although boiling onset predicted in modeling agreed well with the experimental data. An experimentally validated numerical model thus can be an effective tool in both laboratory and clinical HIFU setting. [Work is supported by NIH EB007643 and NSBRI SMST01601.]

\section{2:40}

2pPA6. Numerical simulation of the propagation of three-dimensional helical shock waves in a weakly heterogeneous medium. Regis Marchiano, Francois Coulouvrat (Univ. Pierre et Marie Curie, Institut Jean Le Rond d'Alembert (UMR CNRS 7107), 4 place Jussieu, 75005 Paris, France), and Jean-Louis Thomas (Univ. Pierre et Marie Curie, 75005 Paris, France)

A numerical method for the simulation of 3-D acoustical shock wave propagation through a homogeneous or weakly heterogeneous medium is presented. It is based on a generalization of the KZ equation taking into account weak heterogeneities. The algorithm is based on a spectral treatment of the linear diffraction (angular spectrum), coupled with quasianalytical solutions for the heterogeneous part and for the nonlinear one. This last one solves Burgers' equation with the so-called Burgers-Hayes method using the potential instead of the pressure field. The combination of these several algorithms lead to the development of an efficient software, allowing to solve full 3-D problems for standard personal computers. This software is used to study the dynamics of helical shock waves also called acoustical vortices (AVs) which are the acoustical equivalent of optical vortices. The 3-D helical spatiotemporal wave field is characterized by azimuthal shocks. The dynamics of the so-called topological charge, an intrinsic property of AVs, is studied in the nonlinear regime through different focusing lenses. 
2pPA7. Numerical methods for weak shock propagation and diffraction around a microphone. Victor W. Sparrow (Penn State, Grad. Program in Acoust., 201 Appl. Sci. Bldg., University Park, PA 16802, vws1@ psu.edu)

There are two interrelated goals for this presentation. The first goal is to give an overview of available numerical approaches for weak shock propagation. There are multiple approaches available, including formulations based on the Burgers equation, KZK or NPE equations, the second-order equations of nonlinear acoustics, the Navier-Stokes equations, or the Boltzmann equation. There are also multiple numerical techniques for discretizing each of underlying equations, and while some approaches give special treatment to weak shocks others handle the shocks automatically. The second goal of this presentation is to show recent simulation results for an explosion shock interacting in two dimensions with a microphone. The diffraction of the weak shock around the microphone housing does turn out to be amplitude dependent. It is only a modest effect, increasing for higher amplitudes. This knowledge is important for precise, closerange blast source characterization. In the future, the nonlinear diffraction of weak shocks around transducers also should be investigated for finite amplitude biomedical ultrasound, since the mechanisms for nonlinear diffraction are almost identical for ultrasound and explosion waves. [Work supported by U.S. Army ERDC-CERL.]

\section{Contributed Papers}

3:40

2pPA8. Simulations of converging shocks in water. Veronica Eliasson (Graduate Aerosp. Lab., Caltech, 1200 E. California Blvd., Pasadena, CA 91107, vero@caltech.edu), William D. Henshaw (LLNL, Livermore, CA 94551), and Paul E. Dimotakis (Caltech, Pasadena, CA 91125)

Numerical simulations of weak shocks in water traveling through a convergent geometry were performed. The convergent geometry is surrounded by an elastic solid, which is deformed by the fluid, thereby, generating elastic waves in the solid, which in turn affect the liquid, thus creating a coupled fluid-structure problem. Here, we use the Overture suit, which is a code for solving partial differential equations on curvilinear overlapping grids using adaptive mesh refinement. In particular, we use a multiphysics solver to solve the fluid-structure problem. The Euler equations with a stiffened equation of state are used in the fluid domain and the linear elasticity equations are used in the solid domain. The solutions at the interface between the fluid and the solid are matched using continuity of normal velocities and forces. Preliminary results indicate that the wave speed of the material has a large influence on the behavior of the converging shock. The numerical simulations are also compared to schlieren photographs and pressure measurements obtained from experiments. Results can enhance the design of marine structures with convergent sections subjected to dynamic loading events. [Work supported by ONR.]

\section{3:55}

2pPA9. Comparison of time and frequency domain approaches to simulate propagation of weak shocks. M.V. Averiyanov, P.V. Yuldashev (Faculty of Phys., Moscow State Univ., Moscow 119991, Russia, misha@acs366.phys.msu.ru), Ph. Blanc-Benon (LMFA, UMR CNRS 5509, Ecole Centrale de Lyon, 69134 Ecully Cedex, France 5509), and V.A. Khokhlova (Univ. of Washington, Seattle, WA 98105)

Simulations of acoustic fields using finite-difference methods are performed either in time or frequency domains. A method of fractional steps with an operator splitting procedure is frequently applied to solve nonlinear equations of the evolution type. Either time or frequency domain solvers can be used to calculate different terms in the equation over a propagation grid step. In this work, several algorithms that have been used to simulate quadratic nonlinear term in the Burgers or KZK-type evolution equations are applied to model the propagation of weak shocks. Shock capturing schemes of Godunov type, exact analytic solution with further extrapolation of the waveform over a uniform temporal grid, time-domain conservative schemes, direct modeling in the frequency domain, and asymptotic spectral approach are compared. The parameters of the schemes that would provide the results of the same accuracy, an artificial absorption necessary for stability of the schemes, resolution of shocks, and internal viscosity of the algorithms are discussed. It is shown that the Godunov-type algorithm is better suited to model weak shocks with sufficient accuracy achieved with only three temporal grid points per shock. [Work supported by the RFBR and NIH EB007643 grants.]
4:10

2pPA10. A shock-fitting method for general nonlinear progressive waves. Francois Coulouvrat (Univ. Pierre et Marie Curie, Institut Jean Le Rond d'Alembert (UMR CNRS 7107), 4 place Jussieu, 75005 Paris, France)

Many physical phenomena are concerned with the propagation of weak nonlinear waves that can be modeled under the form of a generalized Burgers equation. Physical examples include nonlinearities that can be either quadratic (nonlinear acoustical waves in fluids or longitudinal waves in solids), cubic (nonlinear shear waves in isotropic soft solids), or nonpolynomial (Buckley-Leverett equation for diphasic fluids, models for car traffic, Hertz contact in granular media). A new weak shock formulation of the generalized Burgers equation using an intermediate variable called "potential" is proposed. This formulation is a generalization to nonquadratic nonlinearities of the method originally proposed by Burgers himself in 1954 for his own equation, and later applied to sonic boom applications by Hayes et al. (1969). It is an elegant way to locate the position of a shock. Its numerical implementation is almost exact, except for an interpolation of Poisson's solution that can be performed at any order. It is also numerically efficient. As it is exact, a single iteration is sufficient to propagate the information at any distance. It automatically manages waveform distortion, formation of shock waves, and shock wave evolution and merging. The theoretical formulation and the principle of the algorithm are detailed and illustrated by various examples of applications.

\section{4:25}

2pPA11. A nonlinear computational method for the propagation of shock waves in ducts: Application to buzz-saw noise. Rasika Fernando, Yann Druon (Airbus France, Acoust. Environment Dept. Turbomachinery Acoust. (EDEA3), 316 route de Bayonne, 31060 Toulouse Cedex 03), Regis Marchiano, and Francois Coulouvrat (Univ. Pierre et Marie Curie, 75005 Paris, France)

A numerical method to compute shock wave propagation in uniform waveguides in 2-D and 3-D is presented. The solution is searched under the form of a modal solution of the Kuznetsov equation for which the modal amplitudes of the analytical linear modes are supposed to vary along the duct axis due to nonlinear interactions between the different modes and frequencies. This finally yields to a differential system on the mode amplitudes, which is solved numerically using a standard Runge-Kutta algorithm for ordinary differential equations after numerical truncation of the modal series. Examples are presented of the nonlinear evolution of the pressure field for a 2-D waveguide. One important 3-D application is the so-called "buzz-saw" noise occurring for high-bypass-ratio turbofan engines, when fan blade tip relative flows exceed the sound velocity. First results on 3-D simulations for nonlinear propagation of a saw-tooth waveform spiraling inside a hard-walled cylindrical will be presented. Extensions of the model to ducts with uniform flows or with liners (walls with impedance) will finally be discussed. 


\section{4:40}

2pPA12. Evaluation of a wave vector frequency domain method for nonlinear wave propagation. Yun Jing and Greg Clement (Dept. of Radiology, Harvard Med. School, Brigham and Women's Hospital, Boston, MA 02155)

In this paper, a wave vector frequency domain method is presented to describe both forward and backward nonlinear wave propagations in acoustic media. A frequency domain nonlinear wave equation is first derived based on the Westervelt equation by taking the Fourier transform in the temporal and spatial domains. Since the model works directly in the frequency domain, arbitrary dispersion relations can be elegantly considered. The nonlinearity is further evaluated by multidimensional autoconvolution of the Fourier transform of the sound pressure. An implicit solution of the equation is proposed by employing the Green's function, and is solved numerically. Comparisons between the numerical simulations and the Burger's solution show good agreements within the shock formation distance. Error study is also carried out to test the efficiency of the model. It is found that the error grows as the initial pressure increases and the spatial resolution decreases. Finally, the present model is compared with a time-domain approach for a practical case, where the diameter of the transducer is $100 \mathrm{~mm}$, the fundamental frequency is $1 \mathrm{MHz}$, and the focus range is $150 \mathrm{~mm}$.

\title{
Session 2pSA
}

\section{Structural Acoustics and Vibration: Emerging Applications of Structural Acoustics}

\author{
Sean F. Wu, Chair \\ Dept. of Mechanical Engineering, Wayne State Univ., Detroit, MI 48202
}

\section{Invited Papers}

\begin{abstract}
2:00
2pSA1. Intrinsic damping models and their role in predicting structural response. Allan D. Pierce (Dept. of Mech. Eng., Boston Univ., Boston, MA 02215, adp@bu.edu) and Adnan Akay (Bilkent Univ., Ankara, Turkey)

In many industries, it is often desired to estimate whether a structure can withstand severe broad-band high-frequency loads. Because the structures have many natural frequencies within the band of interest, the response is critically affected by the damping within the structure. One typically performs finite element model computations with the elastic modulus replaced by a complex number, the ratio of imaginary to real parts being the loss factor, this taken as frequency independent. The crudity of such a model invariably leads to designs that are either overly conservative or not inspiring confidence. The present paper revisits the topic of internal damping from a fundamental standpoint and adopts the viewpoints of [J. J. Thomson (1888), Clarence Zener (1948)], in which "all anelastic phenomena are regarded as the superposition of elementary processes in which the stress relaxes exponentially." The resulting general model involves a continuous distribution over relaxation times. Lumped parameter realizations require that the configuration of the structure be described by the positions of all the mass points, and also by those of a large number of "hidden variables" connected to the mass points by Maxwell elements (spring and dashpot is series). General implications of the model are derived and explained.
\end{abstract}

2:20

2pSA2. Measurement methodologies for the analysis of influence of surface panels and their vibrations on interior cabin noise. Sean Wu (Dept. of Mech. Eng., Wayne State Univ., 5050 Anthony Wayne Dr., Detroit, MI 48202, aa3199@ wayne.edu) and Allan Pierce (Boston Univ., Boston, MA 02215)

In the design of quiet automobile compartments, one analyzes the influence of individual panels and enclosure surface leaks on noise received in a specific region (the location of the driver's ear position). Two recent papers [Hald et al., Internoise (2006); Wolff, Internoise (2007)] suggest the needed measurement process can be shortened using a sound source at the typical listener position, taking measurements over the enclosure surface, and then invoking the principle of reciprocity. The present paper clarifies the analytical discussions of these earlier papers with improved and more nearly rigorous derivations and suggests improved measurement methodologies. One exemplary model considered is that of an arbitrarily shaped cavity with sound absorbing walls that are also moving due to external influences. The boundary condition on the pressure field inside the cavity is shown to be well approximated such that the normal component of the pressure gradient is a superposition of a constant times the pressure itself (locally reacting surface with finite impedance) plus a constant times the portion of the surface velocity that is due to the external sources. The pressure inside the cavity can be solved by Green's function techniques, and the Green's function can be proven to rigorously satisfy reciprocity.

\section{2:40}

2pSA3. Using structural acoustics methods to study new materials. J. D. Maynard (Dept. of Phys., The Pennsylvania State Univ., University Park, PA 16802)

In structural acoustics it is usually necessary to know the vibration properties of the structure under study, and this requires knowing the elastic constants of the materials involved. A relatively recent and powerful method for measuring elastic constants is resonant ultrasound spectroscopy (RUS). Very new and exotic materials for structures often require RUS; the reason is that new materials, when first fabricated, are often available only in very small samples (a few hundred micrometers) or as thin films on substrates, and RUS is the best method for handling such samples. On some occasions samples are fragile or chemically reactive so that they cannot be pol- 
ished into the shapes required by conventional RUS. Thus, it has been necessary to develop a RUS analysis for arbitrarily shaped samples. This has been accomplished by borrowing a method from structural acoustics: the finite element method (FEM). The discussion of the application of FEM to RUS for arbitrarily shaped samples will include bridging the gap between theory (involving matrices as boldfaced symbols without indices in textbooks on FEM) and actual lines of computer code. Applications of the RUS method for materials will also be discussed.

3:00-3:20 Break

$3: 20$

2pSA4. Spatial maps of modal damping from frequency response measurements. J. Gregory McDaniel, Craig Boucher, and Hande Öztürk (Mech. Eng. Dept., Boston Univ., 110 Cummington St., Boston, MA 02215)

A method is proposed for constructing spatial maps of modal damping for viscously damped structures. These maps are intended to be useful in assessing the effectiveness of spatially distributed damping, particularly in complex structures that are damped by a variety of mechanisms. The method uses frequency response measurements and modal analysis to determine the natural frequencies and mode shapes of the damped structure. The damping matrix is estimated directly from the frequency response measurements and is expressed as a sum over connectivity matrices weighted by dashpot constants. This sum is substituted into the well-known approximation for the modal loss factor that results by neglecting off-diagonal elements of the modally transformed damping matrix. The resulting expression quantifies the contribution of local dashpot properties to the modal loss factor, and may be visually presented as a spatial map of damping. A significant advantage of this approach is its independence from a detailed model of the structure or its damping, allowing it to be used for complex structures. The resolution of the map is directly correlating to the number of measurement locations, as will be illustrated by examples. [Work supported by ONR under Grant No. N000140810531.]

2pSA5. A three-dimensional Wiener-Hopf technique for general bodies of revolution, Part II: Substructuring and implementation. Rudolph Martinez and Carina Ting (CAA-Alion Corp., 84 Sherman St., Cambridge, MA 02140)

This paper begins with a brief review of Part I's main ideas and conclusions regarding: (a) Operator-induced symmetry and reciprocity leading to compatibility of the halfplanes of analyticity between the integral equation's non-translational kernel and those of the Fourier-transformed displacements and pressures on the surface of a scattering or vibrating body of revolution; and (b) subsequent spectral factorizations in juxtaposition to those of the classical Wiener-Hopf case for the planar geometry. The spatial and spectral domains in question refer to the arclength variable along the body's generator and to its transform wavenumber, respectively. The analysis described in this Part-II paper brings out some of the additional positive consequences of the pre-symmetrization of the integral equation that governs the response of the coupled fluid and structure. It then applies standard substructuring schemes to extend Part I's formulation to include departures from axisymmetry resulting from generic appendages as discontinuities along the shape's circumferential coordinate. The presentation ends with sample applications of its new 3-D Wiener-Hopf technique to canonical shapes of revolution, and with a summary of the physical insights made possible by the new approach relative to numerical results by boundary elements.

\section{4:00}

2pSA6. Surface wave testing of pavements. Nils Ryden (Eng. Geology, Faculty of Eng., Lund Univ., Box 118, SE-22100 Lund, Sweden)

Pavements are constructed using several layers of materials, and their durability depends on the quality of all of these strata. It is, therefore, valuable to be able to determine the properties of the layers nondestructively. A method is presented for evaluating the thickness and stiffness of multilayered pavement structures from guided waves measured at the surface. In this type of layered structure, the interaction of leaky Lamb waves in the embedded layers generates surface waves corresponding only to certain portions of the guided wave dispersion curves and branches measurable at the pavement surface. To resolve the different mode branches, the wavefield is measured at the surface by using a light hammer as the source and an accelerometer as receiver, generating a synthetic receiver array. The recorded data are transformed to a phase velocity spectrum, which is then inverted to give the layer properties using a global inversion algorithm. The theoretical background along with experimental results of the application to nondestructive testing of pavements will be presented. Ongoing research on noncontact air-coupled measurements is also demonstrated. This opens up the possibility for faster on-the-fly surface wave testing of pavement layers, since surface contact is no longer required.

\section{4:20}

2pSA7. Innovative structural acoustic strategies to reduce sound transmission through lightweight flexible structures. Donald Bliss, Qinxian He, Linda Franzoni, and Cassidy Palas (Mech. Eng. and Mater. Sci., Duke Univ., Durham, NC 27708, dbb@duke.edu)

This paper describes research utilizing structural acoustics in novel ways to cancel the transmission of sound and vibration through multielement flexible barriers. Configurations analyzed include panels with different thickness and elastic modulus in different regions and layered structures connected by an elastic suspension. The purpose of the research is to demonstrate that flexibility and controlled resonant behavior can be used to block sound transmission even when structural damping is very low. Strategies are considered to alter vibrating surface wavenumber spectra to reduce coupling between the structure and the acoustic field. Another approach that can be employed is the utilization of structural wave cutoff with multielement multipath (MEMP) structures. Finally, multiple differentially tuned subsidiary elements acting as resonators can be used to greatly reduce the structural response. Examples of acoustic transmission 
loss through panel barriers using different strategies are presented, and the potential advantages and possible shortcomings of various approaches are evaluated. Possible configurations for layered sound reduction materials are proposed. The work has particular application to the reduction of vehicle interior noise and addresses the need for good acoustic performance of the lighter weight flexible structures that will be used in the future.

TUESDAY AFTERNOON, 19 MAY 2009

EXECUTIVE SALON I/II/III, 2:00 TO 5:30 P.M.

\title{
Session 2pSC
}

\section{Speech Communication and Psychological and Physiological Acoustics: Exploring the Relationship Between Cognitive Processes and Speech Perception (Lecture/Poster Session)}

\author{
Amee P. Shah, Chair \\ Dept. of Speech and Hearing, Cleveland State Univ., Cleveland, OH 44115
}

Chair's Introduction-2:00

Invited Papers

2:05

2pSC1. Perceptual learning and expectations: Cognitive mechanisms in speech recognition. Howard Nusbaum (Dept. of Psych., The Univ. of Chicago, 5848 S. University Ave., Chicago, IL 60637, h-nusbaum@uchicago.edu)

There is substantial plasticity in adult perceptual processing of speech that cannot be accounted for by most theories. Even $1 \mathrm{~h}$ of training on low-intelligibility synthetic speech can improve recognition by 20 percentage points for novel words. Whether speech perception is based on auditory properties or articulatory properties, few theories acknowledge the role of cognitive processes, and some explicitly exclude these mechanisms. Evidence will be examined suggesting perceptual learning of phonetic information in adult listeners involves processes such as attention and working memory. In addition, evidence will be examined that speech perception can be influenced, if not typically guided, by listeners' expectations about speech. In some cases, expectations may be derived by explicit instruction to listeners, and in others, from contextual information. For example, research on talker normalization and perception of sinewave speech has demonstrated that listeners' expectations about the speech signal change perceptual processing. Evidence will be discussed suggesting mechanisms that mediate this effect of expectations and compare this to mechanisms involved in perceptual learning. Perceptual learning may depend on the same kinds of cognitive mechanisms involved in the top-down guidance of perception by expectations and these mechanisms may be necessary for stable phonetic perception. [Research supported by NIDCD.]

\section{2:25}

2pSC2. The role(s) of capacity limitations in speech perception. Alexander L. Francis (Speech, Lang., and Hearing Sci., Purdue Univ., 500 Oval Dr., West Lafayette, IN 47907, francisa@purdue.edu)

This paper reviews some current research exploring the role that capacity limitations play in recognizing speech, particularly in the presence of competing speech. Load theory [Lavie, Trends Cogn Sci 92 (2005)] suggests that perceptual capacity limits the number of objects (or features) that can be processed simultaneously, while cognitive capacity limits rejection of irrelevant information. Functional imaging studies show increased cognitive (working memory) load corresponding to failure of inhibition of (increased activity in) brain regions associated with processing distractor stimuli [de Fockert et al., Science 291 (2001)], whereas increased perceptual load results in decreased activity in distractor-related areas [Rees et al., Science 278 (1997)]. However, all of these studies were carried out in the visual modality, and it is unclear whether the same predictions were obtained in audition or speech perception. A series of experiments in which perceptual and working memory load were varied as listeners identified words produced by one talker while ignoring another provides qualified support for the predictions of load theory and lays the groundwork for subsequent studies of the effects of hearing impairment, cognitive deficits, and perceptual and cognitive training on the perception of speech in competing speech. [Work supported by NIDCD R03DC006811.]

2pSC3. Hearing the forest despite the trees: Perceptual learning of systematic variation in speech. Lynne C. Nygaard (Dept. of Psych., Emory Univ., Atlanta, GA 30322)

A fundamental problem for the understanding of spoken language processing is listeners' robust perceptual constancy in the face of enormous variability in the instantiation of linguistic form. On the one hand, listeners are sensitive to the fine-grained structure of linguistic segments that signal differences among talkers and speaking styles. On the other hand, listeners tolerate large discontinuities in this same fine structure forming robust, perceptually constant linguistic categories. Data from a perceptual learning paradigm addressing both the limits and flexibility of speech perceptual mechanisms will be presented and discussed in light of evidence for perceptual learning of accented speech. In a series of studies, listeners were exposed to accented English under learning conditions in which 
the opportunity to compare across instances varied. At test, generalization and long-term retention of perceptual learning were evaluated. The results suggest that adaptation to lawful variation in speech is fundamentally influenced by the character and structure of the learning experience. These findings suggest cognitive constraints on behavioral and representational plasticity in speech perception and spoken language processing.

\section{3:05}

2pSC4. The nature and time course of the relationship between surface variability and linguistic processing. Amee Shah (Dept. of Health Sci., Cleveland State Univ., 2121 Euclid Ave., MC 429, Cleveland, OH 44115) and Conor McLennan (Cleveland State Univ., Cleveland, $\mathrm{OH} 44115)$

It is becoming increasingly prevalent to study and understand the role of surface information in the process of speech perception and to attempt to accommodate these variables in theories of speech perception. Indeed, theories of speech perception increasingly require an account of the precise circumstances under which surface details affect the ease with which listeners access spoken words. To date, the majority of studies have focused on manipulating various components of surface information (e.g., speaking rate, talker differences, dialect difference, and so on) to determine whether these manipulations matter to the processing task. The present set of studies in our laboratory represents the converse component of this relationship in order to determine whether linguistic complexity can affect or modulate listeners' overt subjective impressions of the surface information. Two different methodologies, including semantic priming and repetition priming, were used manipulate ease of lexical access and determine the role on listeners perception of surface details (foreign accent). Additionally, the time course of the effect of linguistic context on accent rating judgments was studied as it manifested itself differently at different points during perceptual processing. Overall, these studies help understand the complex nature of the relationship between surface variability and linguistic processing.

2pSC5. Influences of auditory object formation on speech perception. Barbara G. Shinn-Cunningham and Dali Wang (Boston Univ. Hearing Res. Ctr., Auditory Neurosci. Lab., 677 Beacon St., Boston, MA 02215)

In common social settings, our ears receive a jumbled mixture of sound coming from different sources in different directions. A normal hearing listener is adept at using the low-level acoustic structure in the sound mixture to organize this cacophony into auditory objects, each corresponding to a distinct talker (i.e., performing auditory scene analysis). Typical listeners are also proficient at focusing on the talker of interest and ignoring distracting objects (i.e., directing selective attention to the desired object). Often, despite the efficacy of object formation and selective attention, portions of an attended object are inaudible, masked by sources that overlap the attended source in time and frequency. In such situations, listeners "fill in" the missing speech and extract a meaningful, whole message from the sound mixture, a process known as "phonemic restoration." This talk will review some recent studies demonstrating that the process of phonemic restoration, which relies on high-level, acquired knowledge about speech structure and meaning, is directly impacted by low-level acoustic attributes that influence auditory grouping. These results demonstrate the inter-connected nature of the high-level processes that allow listeners to communicate in challenging settings.

$$
\text { 3:45-3:55 Break }
$$

\section{3:55—4:25 Panel Discussion}

\section{4:25—4:30 Break}

\section{Contributed Papers}

All posters will be on display from 4:30 p.m. to 5:30 p.m. To allow all contributors an opportunity to see other posters, contributors of odd numbered papers will be at their posters from 4:30 p.m. to 5:00 p.m. and contributors of even numbered papers will be at their posters from 5:00 p.m. to 5:30 p.m.

2pSC6. The learning of lexical tones. Sunjing Ji (Dept. of Linguist., Univ. of Arizona, Douglass Bldg., Rm. 200E, Tucson, AZ 85719, sunjing@email .arizona.edu)

Our understanding of speech perception suggests that categorization is an essential task of our cognitive system. Based on previous research on segment perception, this study explores the questions of what mechanism is involved in the acquisition of lexical tones and whether contour tones are decompositional in the process of cognitive learning. An experiment adopting the statistical training method from Maye et al. (2002) and Hayes-Harb (2007) was conducted on the learning of lexical tones by adult naive tone learners. The results show that statistical distribution of stimuli along a pitch continuum in training affects naive tone learners' formation of level lexical tone categories. In addition, the perception of level tones behaves more like the perception of vowels rather than consonants. Moreover, exposure to statistical training on a level tone continuum alone affects participants' performance in contour tone discrimination. This indicates that participants were able to apply level tone categories to generate new mental categories of contour tones, providing psychological evidence for the auto-segmental argument that contour tones are composed of a sequence of multiple level tones.

2pSC7. Effects of differences in fundamental frequency on cross-formant grouping in speech perception. Robert J. Summers, Brian Roberts (Psych., Sch. of Life and Health Sci., Aston Univ., Birmingham B4 7ET, UK, r.j.summers@aston.ac.uk), and Peter J. Bailey (Dept. of Psych., Univ. of York, Heslington, York YO10 5DD, UK)

In isolated syllables cross-formant perceptual grouping can be promoted by a common fundamental frequency (F0), but this effect is relatively weak [Darwin, Q., J. Exp. Psychol. 33A, 185-207 (1981)]. Few studies have explored the role of F0 in cross-formant grouping using sentences. Threeformant $(\mathrm{F} 1+\mathrm{F} 2+\mathrm{F} 3)$ analog of almost continuously voiced natural utterances were synthesized using a monotonous glottal source $(\mathrm{F} 0=150 \mathrm{~Hz})$. Perceptual organization was probed by presenting stimuli dichotically $(\mathrm{F} 1+\mathrm{F} 2 \mathrm{C}+\mathrm{F} 3$; F2), where $\mathrm{F} 2 \mathrm{C}$ is a competitor for F2 that listeners must resist to optimize recognition. Competitors were created using time-reversed 
amplitude and frequency contours of $\mathrm{F} 2$, and $\mathrm{F} 0$ was manipulated $(\Delta \mathrm{F} 0=$ $\pm 8, \pm 2$, or 0 semitones relative to the other formants). Adding F2C reduced intelligibility for most utterances, and this reduction was greatest overall when $\Delta \mathrm{F} 0=0$. However, as for isolated syllables, the effect of $\Delta \mathrm{F} 0$ was quite small. Competitor efficacy was also somewhat dependent on absolute F0. Manipulation of relative F2C level indicated that competitor efficacy was not due primarily to energetic masking. The modest effect of $\Delta \mathrm{F} 0$, both for sentences and isolated syllables, suggests that cross-formant grouping depends more on other factors, for example common patterns of modulation. [Supported by EPSRC]

2pSC8. The perceptual organization of sine-wave speech under competitive conditions. Brian Roberts, Robert J. Summers (Psych., Sch. of Life and Health Sci., Aston Univ., Birmingham B4 7ET, UK, b.roberts@aston.ac.uk), and Peter J. Bailey (Univ. of York, Heslington, York YO10 5DD, UK)

Speech comprises dynamic and heterogeneous acoustic elements yet is heard as a single perceptual stream, even when accompanied by other sounds. The relative contributions of grouping primitives and of speechspecific factors, for example modulation patterns, to the perceptual coherence of speech are unclear, and the critical acoustical correlates of the latter remain unknown. The parametric manipulations possible with simplified speech, such as sine-wave analogs, make them attractive experimental stimuli to explore this issue. Given that the factors governing perceptual organization are generally revealed only where competition operates, the second-formant competitor (F2C) paradigm was used, in which the listener must resist competition to optimize recognition [Remez et al., Psychol. Rev. 101, 129-156 (1994)]. Three-formant (F1+F2+F3) sine-wave analogs were derived from natural utterances and presented in a dichotic configuration (one ear $=\mathrm{F} 1+\mathrm{F} 2 \mathrm{C}+\mathrm{F} 3$; opposite ear $=\mathrm{F} 2$ ). Different versions of F2C derived from $\mathrm{F} 2$ by manipulating its amplitude and frequency contours were effective at reducing intelligibility except when the frequency contour was constant. Manipulation of relative $\mathrm{F} 2 \mathrm{C}$ level indicated that competitor efficacy was not due primarily to energetic masking. The findings suggest that modulation of the frequency, but not the amplitude, contour is critical for cross-formant grouping. [Work supported by EPSRC.]

2pSC9. Developing audio-visual associations in infancy. Francisco Lacerda, Eeva Klitfors, and Ellen Marklund (Dept. of Linguist., Stockholm Univ., SE-106 91 Stockholm, Sweden, frasse@ @ling.su.se)

To investigate how possible biases in matching of object sizes with sound intensities may be influenced by the ecological relevance of the potential associations, three groups of Swedish infants $(4,6$, and 8 months of age) are being tested in their preferences to match speech or nonspeech sounds of different intensities with visual stimuli showing faces or unanimated objects of different sizes. The infants' audio-visual matching preferences are measured by the cumulative time that the infants' gaze vector was directed to each of the faces and objects simultaneously displayed on the screen, in response to the presentation of an acoustic stimulus (either a speech sound or a nonspeech sound). The results appear to suggest a general trend towards matching of visual prominence with sound intensity, when the ecological relevance of the audio-visual associations was high (i.e., matching speech sounds with faces and nonspeech sounds with unanimated objects) but the pattern seems to be disturbed when only nonecologically relevant matches are possible. [Work carried out within grants from The Bank of Sweden Tercentenary Foundation, Grant No. K2003:0867, MILLE, from EU-NEST Project No. 5010, CONTACT, and from the Knut and Alice Wallenberg Foundation, Grant No. KAW 2005.0115.]

$2 \mathrm{pSC10}$. Voice familiarity helps infants tackle variability in the speech signal. Marieke van Heugten and Elizabeth Johnson (Dept. of Psych., Univ. of Toronto Mississauga, 3359 Mississauga Rd. N., Mississauga, ON, Canada, L5L 1C6, marieke.vanheugten@utoronto.ca)

The acoustic realization of lexical items produced by different speakers can vary greatly. Current research suggests that infants, unlike adults, struggle to cope with this lack of invariance in the realization of words. Although 7.5-month olds are able to recognize words across different utterances when produced by speakers of the same gender with similar voices, they fail to do so when target words produced in a female voice are subsequently produced in a male voice [Houston and Jusczyk (2000)]. Note that all work in this area has used disembodied unfamiliar voices to test infants. In the current study, we ask whether infants might perform better under more ecologically valid conditions, i.e., when tested on familiar rather than unfamiliar voices. Using the headturn preference procedure, infants were familiarized with passages spoken by their mother. During the test phase, they were presented with their father's voice producing isolated repetitions of familiarized target words. Preliminary results suggest that infants may recognize words across different utterances produced by speakers of different genders if they are highly familiar with both the male and female speakers. In other words, infants may handle variability in the realization of words better when tested on familiar rather than unfamiliar voices.

2pSC11. The development of infants' sensitivity to modified spectral tilt: Fricatives, approximants, and vowels. Elizabeth Beach and Christine Kitamura (MARCS Auditory Labs., UWS, Bdg. 5 Bankstown Campus, Locked Bag 1797, Penrith South DC 1797, NSW, Australia)

In early infancy, speech perception is based on innate psychoacoustic thresholds allowing young infants to discriminate a wide range of speech contrasts. However, as infants accumulate knowledge of their native language, they begin attuning to native speech sounds: first vowels around 6 months; then consonants around 9-12 months. Now that hearing-impaired infants are being diagnosed and fitted with hearing aids early in life, there is a need to investigate how speech is amplified in infant hearing aids. This study examined whether infants would benefit from positive, negative, or unmodified spectral tilt. Spectral tilts of $+6 \mathrm{~dB} /$ octave and $-6 \mathrm{~dB} /$ octave (as found in hearing aids) were applied to three speech contrasts (fricatives, approximants, vowels) and in a third condition the contrasts remained unmodified. Normal-hearing 6- and 9-month-olds were tested using an habituation procedure to determine whether positive, negative, or unmodified tilt aids discrimination of the speech contrasts. The results showed 6-montholds benefit when relevant frequency information is amplified, i.e., highfrequency for fricatives and approximants, low-frequency for vowels. Ninemonth-olds, on the other hand, discriminated all contrasts best when unmodified. The developmental change between 6 and 9 months might be explained by infants shifting from an acoustic to a linguistic mode of speech perception. 


\title{
Session 2pSP
}

\section{Signal Processing in Acoustics and Underwater Acoustics: Detection and Classification of Underwater Targets II}

\author{
Patrick J. Loughlin, Cochair \\ Dept. of Bioengineering, Univ. of Pittsburgh, Pittsburgh, PA 15261 \\ Jack McLaughlin, Cochair \\ Applied Physics Lab., Univ. of Washington, Seattle, WA 98105
}

Invited Papers

$1: 30$

2pSP1. Alternative signal processing models for broadband underwater propeller sounds. Les Atlas, Pascal Clark (Dept. of Elec. Eng., Univ. of Washington, Campus Box 352500, Seattle, WA 98195, atlas@u.washington.edu), and Ivars Kirsteins (Naval Undersea Warfare Ctr., Newport, RI)

Underwater propeller sounds often have audibly rhythmic characteristics. These sounds are commonly modeled as a broadband noise carrier multiplied by a rhythmic modulator, where analysis of the modulator wave form can provide information relating to the speed and identity of the source vessel. A natural assumption is to posit a purely periodic modulator, considering the rotary action of the propeller, which is useful for deriving maximum-likelihood estimates of the shaft rate [Lourens and du Preez, J. Ocean. Eng. 23, 4, (1998)]. Indeed, recorded propeller data are unmistakably rhythmic, but the assumption of a strictly periodic or cyclical modulator wave form is questionable. A critical analysis of the periodicity assumption is presented and compared to three alternatives. Maintaining the product form in which the modulator wave form multiplies a random broadband carrier, the possible modulator signals are generalized to include: (1) almost-periodic functions expressible by nonharmonic trigonometric series; (2) multiplicative cascades of possibly complex-valued modulator functions; and (3) stochastic processes in which random state transitions constitute a sense of repeatability or rhythm. In this work, three modulator models are contrasted as estimators of periodicity, using actual propeller sound recordings to validate these estimates. [This work is supported by the Office of Naval Research.]

\section{$1: 50$}

2pSP2. The propagation of noise in a dispersive medium. Leon Cohen (Dept. of Phys., City Univ. of NY, Hunter Coll., 695 Park Ave., New York, 10021)

If noise is generated at a particular region, it will generally propagate and be nonstationary in position and time. We present a phase space approach to understand the propagation of noise in a dispersive medium and to present methods to ascertain when the noise is stationary or quasi-stationary. The damping case is also discussed. In addition, we present approximate methods that allow one to study the statistical properties of the evolution of the noise field in space and time in a relatively simple way. A number of examples will be presented. [Work supported by ONR.]

$$
\text { 2:10 }
$$

2pSP3. Classification of shallow water passive sonar signals using stochastic channel model. Maya Gupta, Hyrum Anderson, and William Mortensen (Dept. of Elec. Eng., Univ. of Washington, Seattle, WA 98195)

This paper addresses the problem of classifying passive sonar signals propagating in shallow water channels. A key problem is that such signals are corrupted by noise and multipath, which we model stochastically in terms of the expected multipath and noise, and the variance of the multipath and noise. We assume that free-field (e.g., deep water) training signals are available. We show that for classification, the accuracy can be improved by not committing to an estimate of the channel or signal, but rather by marginalizing out the channel and noise uncertainty. Specifically, we propose variants of the quadratic discriminant analysis (QDA) classifier and the support vector machine classifier (SVM) that probabilistically account for the unknown channel effects, and which avoid ill-posed deconvolution. The proposed classifiers can work either directly on a time signal or on subband power features. Results on simulated data and real Bowhead whale vocalizations show that we can significantly improve classification performance over traditional methods over a range of signal-to-noise ratios. In addition, we will discuss the case where the classification decision can be made by multiple cooperating receivers, such as in a sonar network.

$$
\text { 2:30 }
$$

2pSP4. Finding physically motivated classification features using finite element models. Jack McLaughlin and Lane M.D. Owsley (Univ. of Washington, Appl. Phys. Lab., Box 355640, 1013 NE 40th St., Seattle, WA 98105)

Finite element models (FEMs) for simple shapes such as spheres, cylinders and pipes can be used to uncover potential features for classification by sonar systems. Analyses were conducted of the simulated, bistatic returns over a wide range of aspects using FEMs for a solid steel cylinder and a cement pipe all in free space. Salient characteristics noted in this way were then sought in field data collected 
from the same or similar objects during the SAX04 experiment and two other data collections conducted in a test pond at the Naval Surface Warfare Center in Panama City, Florida. Characteristics robust enough to appear in both simulated data and field data are candidates to serve as classification features for similarly shaped objects. It is found that FEMs can provide important pointers to classification features that are closely tied to the physics of reflection. As such, the variability of these features in the face of object burial and multipath can be more easily assessed than statistical spectral features. [This work was supported by the U.S. Navy Office of Naval Research.]

2:50

2pSP5. Kirchhoff scattering from underwater targets modeled as an assembly of triangular facets. Ahmad T. Abawi (HLS Res., Inc., La Jolla, CA)

Scattering from underwater targets is a challenging problem, mainly because many of the techniques developed in scattering theory are for radar applications and, therefore, appropriate only in free space. Underwater scattering occurs in a waveguide, which in most cases requires a modification if not a reformulation of the methods developed for scattering from targets in free space. The most accurate solution of scattering from a target in a waveguide can be obtained by solving the wave equation in an environment that contains the waveguide and the target and simultaneously imposing boundary conditions on the boundaries of the waveguide and on the surface of the target. However, this technique is numerically intensive making it impractical in most cases of interest. As a means of offering a more practical solution, we show how to solve the scattering problem from a target in a waveguide by (1) modeling it as an assembly of triangular facets, (2) computing scattering from each facet analytically using the Kirchhoff approximation for each set of incident and scattered plane waves, and (3) combining the solutions coherently. We compare this solution with those obtained using more accurate methods such as the virtual source technique.

\section{3:10-3:20 Break}

\section{Contributed Papers}

\section{$3: 20$}

2pSP6. Change detection deconfliction process for sonar clutter items. John Dubberley and Marlin Gendron (Naval Research Lab. Code 7441, Stennis Space Ctr., MS 39529, john.dubberley@nrlssc.navy.mil)

When resurveying a seafloor area of interest during change detection operations, an automated method to match found bottom objects with objects detected in a previous survey allows the surveyor to quickly sort new objects from old objects. Here we will demonstrate a software system that accomplishes change detection. The change detection system contains modules for automatic object detection by geospatial bitmap technique, object collocation, feature matching using shadow outlining, scene matching by control point matching, and visualization and filtering capabilities. Emphasis will be placed on the new elements of the system, namely, shadow outlining and optional spatial filtering.

\section{3:35}

2pSP7. Processing techniques applied to underwater target echoes and their relationships. Brian H. Houston (Naval Res. Lab., Code 7130, 4555 Overlook Ave., Washington, DC 20375-5320, brian.houston@nrl.navy.mil), Joseph A. Bucaro (Naval Res. Lab., Washington, DC 20375-5320), Larry Kraus (Global Strategies (North America), Crofton, MD 21114), Harry J. Simpson (Naval Res. Lab., Washington, DC 20375-5320), and Timothy J. Yoder (Global Strategies (North America), Crofton, MD 21114)

Interest in acoustic scattering from underwater unexploded ordnance (UXO) has been increasing because of the growing need for sonar technology able to detect submerged UXO and to efficiently separate these detections from those due to natural and man-made clutter. Recent efforts range from laboratory and numerical studies seeking to understand the basic structural acoustic echo formation processes and the environmental effects on them to field exercises determining how well a particular detection /identification technology performs. This paper discusses several important scattering-based constructs, which have been utilized in both the basic UXO studies and the technology exercises as well. They include: (1) $\sigma(\omega, \theta)$, the frequency/angle-dependent scattering cross section; (2) standard imagery, oftentimes implemented with synthetic aperture arrays; (3) reflection tomography imaging; and (4) supersonic imaging using holographic techniques. Without a clear appreciation for the differences among the constructs, it is difficult to properly relate the results of the various studies. We have measured the broadband scattering from several underwater UXO targets using laboratory, rail-based, and AUV systems and processed the data using the above constructs. The use of a common, high quality UXO scattering data base in this process helps clarify the differences and relationships among the constructs. [Work supported by SERDP and ONR.]

\section{3:50}

2pSP8. Bistatic synthetic aperture sonar images of penetrable cylinders. Christopher Dudley and Philip L. Marston (Phys. and Astron. Dept., Washington State Univ., Pullman, WA 99164-2814)

Bistatic synthetic aperture sonar (SAS) and acoustic holographic images and monostatic backscattering by penetrable tilted cylinders in water were investigated. Refracted rays internally reflected from the cylinder's backside often dominated the scattering. Ray theoretic models predict scattering enhancements for cylinders made of some isotropic and anisotropic materials. Experiments involved solid cylinders and liquid-filled cylindrical shells of polymers and fiberglass. The experiments used transient insonification and $k a$ in the range of $9<k a<40$, where $k a$ is a product of the acoustic wave number and the cylinder radius. Bistatic measurements used a hydrophone scanned along a line. Transversely isotropic fiberglass rods produced rich features in the images and in the backscattering plotted as a function of tilt and time. The timing of some features could be explained from a ray model based on properties of quasi-shear and quasi-longitudinal waves computed from the stiffness matrix measured for the fiberglass. Bright features for solid cylinders and liquid-filled shells were typically associated with refracted waves having phase velocities less than the speed of sound in water. For some tilt angles, radiated wavefronts were relatively flat producing enhanced farfield scattering. [Work supported by ONR.]

\section{4:05}

2pSP9. Bistatic synthetic aperture sonar and acoustic holographic imaging of a tilted circular elastic plate. Neil Tuazon and Philip L. Marston (Phys. and Astron. Dept., Washington State Univ., Pullman, WA 99164-2814, marston@wsu.edu)

Circular plates have axial symmetry and are sometimes used as components of items relevant to applications of sonar to detection and classification. Measurements of the backscattering spectrum as a function of tilt angle for an isolated circular plate reveal bright features associated with the excitation of compressional and flexural waves on the plate. Some of the features were previously found relevant to the interpretation of acoustic holographic images based on 2-D hydrophone scans close to a circular plate [B. T. Hefner and P. L. Marston, ARLO 2(1), 55-60 (2001)]. In the present research, bistatic synthetic aperture sonar (SAS) and holographic images were constructed from line scans of a hydrophone at a large distance from 
the plate using a method previously summarized [K. Baik, C. Dudley, and P. L. Marston, J. Acoust. Soc. Am. 121, 3203 (2007)]. Because of elastic responses, the images are bright even when the receiver scan line does not intersect specular reflections from the plate. The radiation by the transient elastic waves excited on the plate can significantly increase the contrast of SAS and holographic images. [Work supported by ONR.]

\section{$4: 20$}

2 pSP10. Optical simulation of bistatic specular reflection by a rigid cone on a flat surface. Anthony R. Smith, Matthew D'Asaro, and Philip L. Marston (Phys. and Astron. Dept., Washington State Univ., Pullman, WA 99164-2814, asmith8@mail.wsu.edu)

To gain insight into the high-frequency scattering of sound by coneshaped objects near flat reflecting surfaces, a small conical mirror was placed on a flat horizontal mirror and illuminated by a tilted laser beam. The symmetry axis of the cone was vertical. Light scattering patterns were recorded by imaging with a camera on a white vertical screen that intersected the scattering. Bright regions on the screen were associated with specular reflections from the cone and from the flat mirror. To determine which contributions depended on the presence of the flat mirror, the screen was also imaged with the cone on a nonreflecting surface. The patterns were recorded with the screen placed sideways relative to the laser beam as well as in front of the cone and behind the cone. The cone half-angle was 45 deg but the grazing angle of the laser beam could be changed. The bright patterns were described by a geometric analysis of bistatic reflection previously summarized [P. Marston, J. Acoust. Soc. Am. 124, 2584 (2008)] modified so as to allow for images caused by the flat mirror. The pattern for a vertical circular cylinder on a mirror was also studied. [Research supported by ONR.]

$$
4: 35
$$

2pSP11. Modelled Doppler and range resolutions of active pulses in simulated environments. Andrew Holden (Dstl Farnborough, Ively Rd., Farnborough, Hampshire, GU14 0LX, UK)

The ability of an active sonar waveform to simultaneously estimate the range and velocity resolution of a target can be predicted by the wideband ambiguity function. Although this gives good insight into the capabilities of different pulse types, it does not replicate a real active sonar where a transmitted pulse is propagated through an environment and the received pulse is cross-correlated with a number of frequency and time shifted replicas of the transmitted pulse. In order to predict this a ray trace model is used to calculate the travel time and received amplitude of each ray path from the transmitting sonar to a target and back to include seabed and sea surface scattering where complex broadband pulse types can be modelled using a sub-band approach. This allows the determination of Doppler and range resolution for a variety of pulse types including pulse trains of LFM pulses in simulated environments, especially highly reverberate shallow water environments.

\title{
Session 2pUW
}

\section{Underwater Acoustics and Acoustical Oceanography: Session in Honor of Ralph Goodman and His Contributions to the Acoustics of Bubbles and Other Works}

\author{
Jerald W. Caruthers, Cochair \\ Dept. of Marine Sciences, Univ. of Southern Mississippi, Stennis Space Center, MS 39529 \\ Kenneth E. Gilbert, Cochair \\ National Ctr. for Physical Acoustics, Univ. of Mississippi, University, MS 38677 \\ Steve Stanic, Cochair \\ Naval Research Lab., Stennis Space Center, MS 39529 \\ Chair's Introduction-1:00
}

Invited Papers

1:05

2pUW1. Ralph Goodman, builder of laboratories. Samuel W. Marshall (P.O. Box 668, White Stone, VA 22578, swmiii@ yahoo.com)

Ralph had an extraordinarily successful career in building programs and laboratories. He started with an overall vision of what was to be built while determining the principle obstructions. He carefully avoided early details which would most often be overtaken by events. In addition, he was an eminent scientist whose acumen in a number of technical areas permitted him breadth of very constructive technical leadership. Besides a history of how Ralph got into underwater acoustics and his first major position in management, some personal anecdotes will be presented to emphasize how he successfully dealt with people and with some very difficult situations in this and subsequent undertakings. 
2pUW2. The Italian connection: Ralph Goodman's close ties to NATO over 45 years. Finn B. Jensen (NATO Undersea Res. Ctr., 19126 La Spezia, Italy, jensen@nurc.nato.int)

Ralph's enduring and very intense professional relationship with SACLANTCEN (now NURC) in La Spezia, Italy, started in September 1961 when he joined the Centre as a young scientist. He worked in Italy for two years publishing on "convergent zone propagation" and "reverberation from smooth ocean floors." This was also the time of his first sea trial, which on Ralph's watch nearly led to the ship running aground. His love for life in Italy and the NATO Centre as such led to many visits in the coming years. He returned in 1981 to become the first American director of SACLANCEN. His primary task was to plan and undertake the construction of a new NATO research vessel named Alliance, a task which involved many crucial decisions about ship specifications and performance, plus guidance to the in-house ship building team, and excruciating negotiations with NATO agencies to secure the funding. Ralph's broad scientific background and superb people management skills ensured the success, and his "yacht" is still one of the best research vessels around, here 20 years later. Ralph's tenure as director lasted an unprecedented 6 years and he left in 1987 having secured SACLANTCEN a top-rated seagoing capability for years to come.

$1: 45$

2pUW3. Coupled ocean-acoustics studies at Navy and NATO laboratories: The legacy of Ralph Goodman. Steve A. Piacsek, Charlie N. Barron (Oceanogr. Div., Naval Res. Lab., Stennis Space Ctr., MS 39529, piacsek@nrlssc.navy.mil), and Michael Porter (HLS, Inc., La Jolla, CA 92037)

A brief history of collaborations between ocean and acoustic modelers is given under the directorships of Ralph Goodman at NORDA and SACLANT, and of continuing coupled modeling studies at NRL built on previous studies he sponsored. At NORDA the Numerical Modeling Division consisted of an acoustic and an ocean branch, one of the first known instances of such close-knit administrative units for ocean and acoustic modelers. At SACLANT, close collaborations were strongly encouraged by Ralph Goodman and the respective group leaders. The results of these studies eventually appeared among the first published papers on the effects of mixed layer and Gulf Stream properties on surface-duct propagation (Computational Acoustics: Ocean-Acoustic Models and Supercomputing (North Holland Press, 1990); Oceanography and Acoustics: Prediction and Propagation Models (AIP Press, 1994). At NRL, these studies were restarted recently: evaluation of sonic layer depth relative to mixed layer depth [JGR 113 (2008)]; the acoustic impact on marine mammals [AGU Ocean Sciences Meeting (2006)]; and the acoustic impact of propagating internal solitons [IEEE Journal of Ocean Engineering (2007)]. Examples will be given from some of these studies to show progress in the field.

\section{2:05}

2pUW4. Ralph Goodman and Hamiltonian acoustics. W. A. Kuperman (Marine Physical Lab., Scripps Inst. of Oceanogr., UC San Diego, La Jolla, CA 92093-0238)

Much of the underwater acoustic community that has worked with ray theory starts from the eikonal-ray equations. Since these equations, which are solved numerically, can be derived from a high-frequency asymptotic expansion, Fermat's/Hamilton's principle is typically bypassed. Historically, the introduction of the Hamiltonian formulation of underwater acoustics ray has come from scientists with a background in classical physics and/or from researchers whose goals were to study complicated environments, chaos, etc., using the well developed formalism of Hamilton's equations and perturbation theory. Mostly, these latter applications go back to the 1980s. Among Ralph Goodman's earliest work in underwater acoustics is a paper [R. Goodman and R. B. Duykers, J. Acoust. Soc. Am. 34, 960-962 (1961)] in which he derived the Hamiltonian for convergence zone rays in the harmonic oscillator approximation and then solved the equations by inspection. This paper must be one of the earliest applications of the Hamiltonian formulation to underwater ray acoustics and a part of the beginning of an illustrious career in the underwater acoustics community.

\section{2:25}

2pUW5. Bubble research with Ralph Goodman at Penn State. Kenneth E. Gilbert (Natl. Ctr. for Phys. Acoust., Univ. of Mississippi, University, MS 38677)

During the years 1992-1998 at Penn State, I collaborated closely with Ralph Goodman on scattering from the near-surface oceanic bubble layer. Ralph's enthusiasm, physical insight, and encyclopedic knowledge of at-sea experiments proved to be invaluable. I will outline the research and discuss Ralph's contributions to graduate theses and scientific papers that were published. I will also give some personal observations on what it was like collaborating with Ralph.

\section{2:45}

2pUW6. Extending bubble measuremenets below $20 \mu \mathrm{m}$ : in memory of Ralph Goodman's contributions. Helen Czerski and David Farmer (The Graduate Sch. of Oceanogr., Univ. of Rhode Island, S Ferry Rd., Narragansett, RI 02882)

No matter which aspect of underwater acoustics we worked on, conversations with Ralph Goodman invariably added insight and often motivated new lines of investigation. This was certainly true of our interest in measuring bubble size distributions. We discuss the extension of acoustical measurement of bubbles to radii below $20 \mu \mathrm{m}$, a topic of particular relevance to the study of optical scatter in the ocean. Extending the frequency range of a resonator to $1 \mathrm{Mhz}$ would in principle allow measurement down to $\sim 3 \mu \mathrm{m}$, but significant challenges arise due to the confounding effects of geometric scatter from larger bubbles. This problem becomes greater for measurements of smaller bubbles, requiring a careful analysis of the accuracy limits imposed on the inversion procedure. Preliminary steps along this path are discussed with examples drawn from recent field measurements. 
2pUW7. Anomalous dispersion and pulse propagation in oceanic bubble clouds. Frank S. Henyey (Appl. Phys. Lab., Univ. of Washington, 1013 NE 40th St., Seattle, WA 98105, frank@apl.washington.edu)

During a planning meeting for the Scripps pier bubble experiment, Ralph Goodman suggested we apply the Kramers-Kronig relations to bubble cloud data. By "we," I assumed he meant that I should do it. I applied the Kramers-Kronig relations to David Farmer's Scripps pier data from his modified Medwin resonators. This allowed a determination on which data had lower accuracy than the rest. Using the high accuracy data, I constructed a model of the complex sound speed. No formula relating bubble size distributions was needed. This model has anomalous dispersion in a frequency band that nearly coincides with a region in which the group speed is higher than the sound speed in bubble-free water. Summerfeld's theorem prohibits any signal from traveling that fast. The consequences for pulse propagation within bubble clouds was determined, using pulses having the same frequency spectrum as transmitted by Jerry Caruthers. The predicted nature of the received pulse is only partially described by Brillouin's studies, done before computers existed.

\section{3:40}

2pUW8. Conversations with Ralph about bubbles in the surf zone. Grant B. Deane (Marine Physical Lab., Scripps Inst. of Oceanogr., UCSD, La Jolla, CA 92093-0238, gdeane@ucsd.edu)

Ralph Goodman made many contributions to the field of oceanic bubbles. This talk will focus on conversations with Ralph about bubbles in the surf zone. Questions that Ralph asked about surf zone bubbles included: where are they, and what are their acoustical properties? These fundamental questions will be examined in the light of Ralph's own experimental and theoretical work, with some contributions from the author. [Work supported by the Office of Naval Research.]

\section{4:00}

2pUW9. Ralph Goodman's recent contributions on oceanic bubbles. Jerald W. Caruthers (Dept. of Marine Sci. Univ. of Southern Mississippi, 1020 Balch Blvd., Stennis Space Ctr., MS 39529, jerald.caruthers@usm.edu) and Steve Stanic (Naval Res. Lab., Stennis Space Ctr., MS 39529)

Many of us are never too old to have an esteemed mentor such as Ralph Goodman. We have for the past decade and a half had the pleasure and privilege of conducting research on oceanic bubbles with his help and advice. Over this period, bubble research has been his main focus. It is appropriate that we highlight his leadership and contributions with us on oceanic bubbles. This presentation will provide snapshots of a number of publications and presentations on bubbles since 1993. [Work supported by ONR and NRL.]

\section{Contributed Papers}

$4: 20$

2pUW10. Shallow water horizontal signal coherence measurements with use of mobile acoustic sources to create synthetic aperture arrays. Philip Abbot, Ira Dyer, and Chris Emerson (OASIS, Inc., 5 Militia Dr. Lexington, MA 02421)

Horizontal signal coherence length was measured in shallow water using a reciprocal synthetic aperture array created from multiple signals radiated by a mobile acoustic source, and a slowly drifting omnidirectional receiver. The tests were conducted in September, 2008, in shallow water $(125 \mathrm{~m}$ depth) of the South East China Sea. Source and receiver depths were $61 \mathrm{~m}$. Twelve hyperbolic FM slides per minute were transmitted while the source moved horizontally at $2.5 \mathrm{~m} / \mathrm{s}$, maintaining a nearly constant range of $\sim 6 \mathrm{~km}$ to the receiver. Received signals were matched-filter processed, phasecorrected to form an equivalent linear array, then coherently combined using a synthetic aperture time delay beamformer. The beamformer output signal gain versus the number of elements in the array, $N$ (using $2 \leqslant N \leqslant 512$ transmissions), was compared to the theoretical limit $(20 \log , N)$. The coherence length, defined to be $L / \lambda$ at a point $3 \mathrm{~dB}$ down from the limit, was determined. At $900 \mathrm{~Hz}$, the correlation length was $L / \lambda \approx 40(N=7$, over 40 s). At $600 \mathrm{~Hz}$, it was $L / \lambda \approx 10(N=3$ over $8 \mathrm{~s})$. The degradation at $600 \mathrm{~Hz}$ is due to substantially smaller signal-to-noise levels at the element level. Partial coherence is observed at both frequencies, even at $N=512$.

\section{$4: 35$}

2pUW11. Attenuation measurements across surface-ship wakes and computed bubble distributions and void fractions: A tribute to the contributions by Ralph Goodman on oceanic bubbles. Steve Stanic (Naval Res. Lab., Stennis Space Ctr., MS 39529, Steve.Stanic@nrlssc.navy.mil), Jerald Caruthers (Univ. of Southern Mississippi, Stennis Space Ctr., MS 39529), Ralph Goodman (Deceased), Edgar Kennedy, and Bob Brown (Naval Res. Lab., Stennis Space Ctr., MS 39529)
A series of three CW-pulsed signals were transmitted across a surface ship wake as the wake aged. Each transmission contained a set of four 0.5ms-long pulses that ranged over frequencies from 30 to $140 \mathrm{kHz}$ in $10-\mathrm{kHz}$ steps. The acoustic attenuation across wakes that were due to varying bubble-size densities within the wakes were determined experimentally. From those data, estimates of the bubble densities as functions of the speed of the wake-generating ship, the wake's age, and acoustic frequency were calculated. From the bubble-density results, power-law fits and void fractions are calculated. The attenuation measurements were taken at $7.5-\mathrm{m}$ intervals behind the wake-generating ship and continued for about $2 \mathrm{~km}$. The experiment was run for wakes generated at ship speeds of 12 and $15 \mathrm{knot}$ wakes. The bubble densities were observed to have power-law forms with varying parameters with the strongest, for early ages, having an exponent of -3.6 and a void fraction of $4 \times 10^{-7}$, and with both diminishing for older wakes, as might be expected. This work was Ralph Goodman's last contribution in underwater acoustics. [Work supported by the Naval Research Laboratory Program Element 62435N and the Office of Naval Research Code 321OA.]

\section{4:50}

2pUW12. Observations and theory of dispersion in rip currents at the Scripps Pier Bubble Experiment of 1997. Jerald W. Caruthers (Dept. of Marine Sci. Univ. of Southern Mississippi, 1020 Balch Blvd., Stennis Space Ctr., MS 39529, jerald.caruthers@usm.edu), Steve Stanic (Naval Res. Lab., Stennis Space Ctr., MS 39529), Jorge C. Novarini (Planning System, Inc., Long Beach, MS 39560), and Frank Henyey (Univ. of Washington, Seattle, WA 98105)

Two of the authors (Caruthers and Stanic), with help and advice from Ralph Goodman, were instrumental in planning and conducting the 1997 Scripps Pier Bubble Experiment. Ralph's participation was invaluable in making this effort a success. A number of institutions were involved in the 
overall experiments, but the main experiments, conducted by the Naval Research Laboratory, centered on what was termed the "delta frame" [Caruthers et al., J. Acoust. Soc. Am., 106, 617-625 (1999)]. The delta frame was a triangle $10 \mathrm{~m}$ on a side with sources at two apexes and eight hydrophones distributed along the arms. Eight frequencies ( 39 to $244 \mathrm{kHz}$ ) were propagated from the two sources. The NRL personnel and other participants in the experiment published a number of detailed measurements and analyses. In the delta frame experiments, a significant amount of the data was relevant to sound-speed-dispersion analyses. Ralph was always keenly interested in dispersion, but we never fully analyzed, nor published those observations. With that oversight and regret in mind that the relevant analyses were not conducted during Ralph's lifetime, we have revisited that data in his honor. [Work supported by ONR and NRL.]

TUESDAY AFTERNOON, 19 MAY 2009

ALEXANDERS LOUNGE, 2:45 TO 3:45 P.M.

\title{
Meeting of Accredited Standards Committee (ASC) S1 Acoustics
}

\author{
P. Battenberg, Chair S1 \\ Quest Technologies, Inc., 1060 Corporate Center Dr., Oconomowoc, WI 53066-4828 \\ R. J. Peppin, Vice Chair S1 \\ Scantek, Inc., 6450 Dobbin Road, \#A, Columbia, MD 21045
}

\begin{abstract}
Accredited Standards Committee S1 on Acoustics. Working group chairs will report on the status of standards currently under development in the areas of physical acoustics, electroacoustics, sonics, ultrasonics, and underwater sound, etc. Consideration will be given to new standards that might be needed over the next few years. Open discussion of committee reports is encouraged.

People interested in attending the meeting of the TAGs for ISO/TC 43 Acoustics and IEC/TC 29 Electroacoustics, take note - those meetings will be held in conjunction with the Standards Plenary meeting at 9:00 a.m. on Tuesday, 19 May 2009.

Scope of S1: Standards, specifications, methods of measurement and test, and terminology in the field of physical acoustics, including architectural acoustics, electroacoustics, sonics, and ultrasonics, and underwater sound, but excluding those aspects of which pertain to biological safety, tolerance and comfort.
\end{abstract}

TUESDAY AFTERNOON, 19 MAY 2009

DIRECTORS ROOM, 4:00 TO 5:00 P.M.

\section{Meeting of Accredited Standards Committee (ASC) S12 Noise}

\author{
R. D. Hellweg, Chair S12 \\ Hellweg Acoustics, 13 Pine Tree Rd., Wellesley, MA 02482 \\ W. J. Murphy, Vice Chair S12 \\ NIOSH, 4676 Columbia Pkwy., Cincinnati, OH 45226
}

\begin{abstract}
Accredited Standards Committee S12 on Noise. Working group chairs will report on the status of noise standards currently under development. Consideration will be given to new standards that might be needed over the next few years. Open discussion of committee reports is encouraged.

People interested in attending the meeting of the TAGs for ISO/TC 43 Acoustics and IEC/TC 29 Electroacoustics, take note - those meetings will be held in conjunction with the Standards Plenary meeting at 9:00 a.m. on Tuesday, 19 May 2009.

Scope of S12: Standards, specifications, and terminology in the field of acoustical noise pertaining to methods of measurement, evaluation and control, including biological safety, tolerance and comfort, and physical acoustics as related to environmental and occupational noise.
\end{abstract}

